\title{
Triterpenoids
}

Cite this: Nat. Prod. Rep., 2013, 30 1028

\author{
Robert A. Hill* and Joseph D. Connolly
}

Covering: 2011. Previous review: Nat. Prod. Rep., 2012, 29, 780-818.

This review covers the isolation and structure determination of triterpenoids including squalene derivatives, lanostanes, holostanes, cycloartanes, cucurbitanes, dammaranes, euphanes, tirucallanes, tetranortriterpenoids, quassinoids, lupanes, oleananes, friedelanes, ursanes, hopanes, onoceranes and saponins; 308 references are cited.
Received 16th April 2013

DOI: $10.1039 / c 3 n p 70032 a$

www.rsc.org/npr appeared describing the triterpenoids isolated from Anemone raddeana,$^{\mathbf{1 0}}$ Poria $\operatorname{cocos},{ }^{\mathbf{1 1}}$ Lantana $^{\mathbf{1 2}}$ and Simaba ${ }^{\mathbf{1 3}}$ species and Pinaceae $^{\mathbf{1 4}}$ and Meliaceae ${ }^{\mathbf{1 5}}$ families. Triterpenoid saponins show a range of biological activities ${ }^{\mathbf{1 6}}$ and this has generated interest in their biosynthesis ${ }^{17}$ and improvement of yields from natural sources. ${ }^{18}$ Reviews covering triterpenoid saponins from Camellia ${ }^{19}$ and Polygala ${ }^{20}$ species and the Theaceae $^{21}$ and Caryophyllaceae and Illecebraceae ${ }^{22}$ families have appeared.

\section{The squalene group}

15-Dehydroxythyrsenol A 1, prethyrsenol A 2 and 13-hydroxyprethyrsenol A $\mathbf{3}$ are new cytotoxic squalene derivatives from Laurencia viridis. ${ }^{23}$ The related compounds 22-hydroxy15(28)-dehydrovenustatriol 4, secodehydrothyrsiferol 5, iubol 6 and 1,2-dehydropseudodehydrothysiferol 7 have also been isolated from Laurencia viridis by the same group. ${ }^{24}$ Squalene1,10,24,25,30-pentol $\mathbf{8}$, which shows moderate anti-<smiles>[R]C(C[C@H]1O[C@H]([C@]2(C)CC[C@@H](Br)C(C)(C)O2)CC[C@]1(C)O)C(=O)[C@](O)(CO)CC[C@@H](O)[C@]1(C)CC[C@@H](C(C)(C)O)O1</smiles><smiles>C=C(CC[C@H](O)C1(C)CCC(C(C)(C)O)O1)C1CC[C@H]2O[C@H](C3(C)CCC(=O)C(=O)O3)CC[C@]2(C)O1</smiles>

mycobacterial activity, has been reported from the leaves and twigs of Rhus taitensis. ${ }^{25}$ The biochemistry and molecular biology of squalene has been reviewed. ${ }^{26}$ 


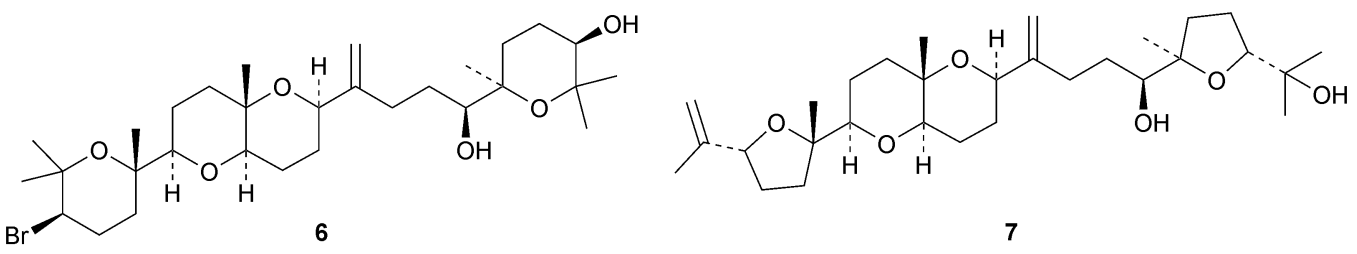<smiles>C/C(=C\CC/C(C)=C/CC/C(C)=C/C/C=C/C(C)(O)C/C=C(\C)CCC=C(CO)CO)CO</smiles>

\section{The lanostane group}

Schiglauzic acid $\mathbf{9}$ and schiglaucyclozic acid $\mathbf{1 0}$ are new lanostanes from the stems of Schisandra glaucescens. ${ }^{27}$ The structures of both compounds were confirmed by X-ray analyses. The three lanostanes 11, 12 and 13, from the leaves of Abies spectabilis, are accompanied by the mariesane derivative $\mathbf{1 4}$ and the $18(13 \rightarrow 17)$-abeo-lanostane $15 .^{28}$ The rearranged lanostane 16 and 24,25,26-trihydroxylanost-7-en-3-one 17 have been isolated from Abies nephrolepis. ${ }^{29}$ The tetradecanoyl ester $\mathbf{1 8}$ is a constituent of Euphorbia sapinii. ${ }^{30}$

Bioactive lanostane derivatives from fungi include 19 and 20 from Poria $\operatorname{cocos}^{31}$ and 24,25,26-trinor-3-oxolanosta-7,9(11)dien-24-oic acid $21^{32}$ and methyl ganoderate A acetonide 22 and butyl ganoderate $\mathrm{H} \mathbf{2 3}^{\mathbf{3 3}}$ from Ganoderma lucidum. The epoxyganoderic acid $\mathbf{2 4}$ is also a constituent of Ganoderma lucidum. ${ }^{34}$ The biological properties of triterpenoids from Poria $\operatorname{cocos}^{35}$ and Ganoderma lucidum $^{36}$ have been reviewed.

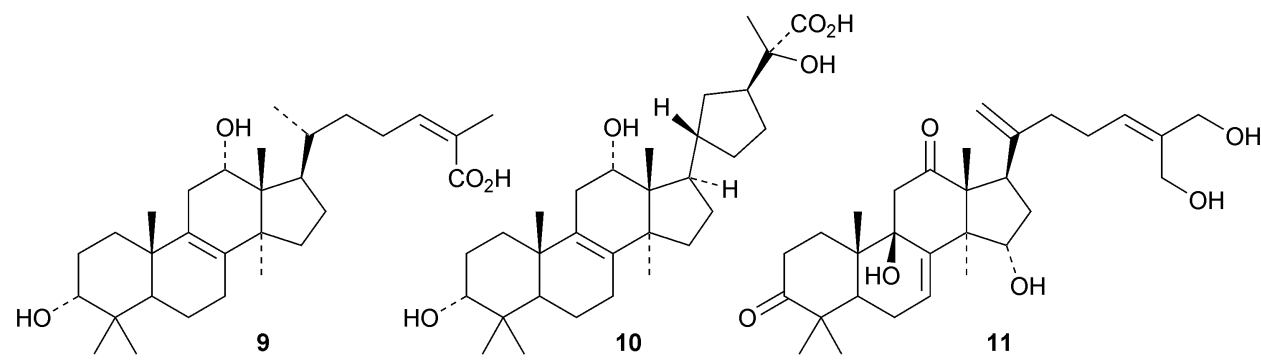<smiles>C=C(CC(=O)O)CC(=O)CC(C)[C@H]1CC[C@H]2C3=CCC4C(C)(CCC(O)C4(C)C)C3=CC[C@]21C</smiles>

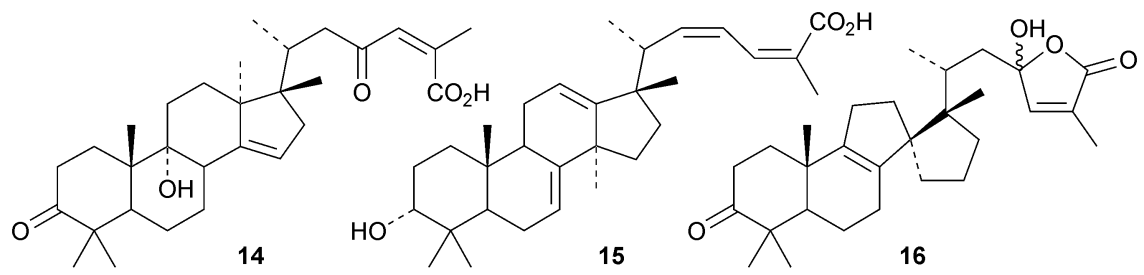

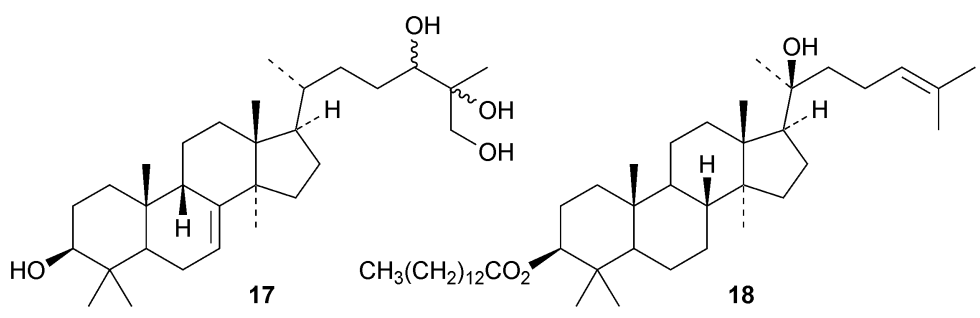




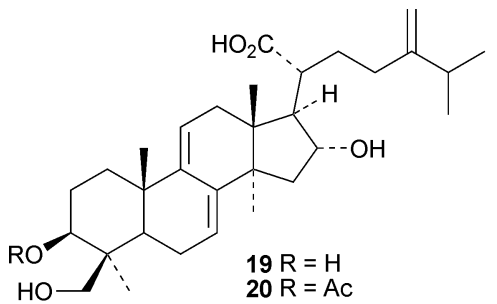<smiles>CCCC(C)[C@H]1CC[C@]2(C)C3=CCC4C(C)(C)C(=O)CC[C@]4(C)C3=CC[C@]12C</smiles><smiles>CCC(=O)C[C@]1(C)C2=C3[C@H](CC4C(C)(C)C(=O)CC[C@]34C)OC(C)(C)OC2C[C@@H]1C(C)CC(=O)CC(C)=O</smiles>

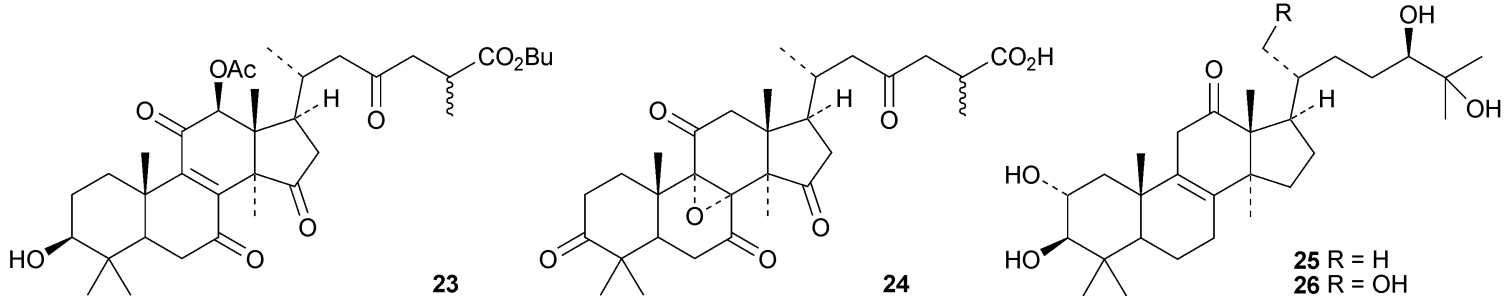

Fasciculols H 25 and I 26 are constituents of the Chinese mushroom Naematoloma fasciculare. ${ }^{37}$ The entomopathogenic fungus Hypocrella sp. BCC 14524 is the source of the lanostanes hypocrellols A-G 27-33. ${ }^{38}$ Xylariacins A 34, B 35 and C 36 have

been isolated from Xylarialeum sp. A45, an endophytic fungus isolated from Annona squamosa. ${ }^{39}$ Inonotsutriols D 37 and E 38 have been reported from the white rot fungus Inonotus obliquus. $^{40}$

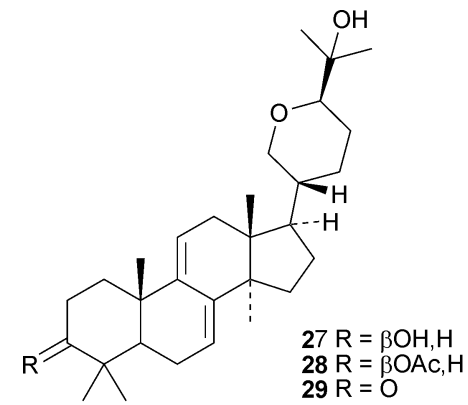

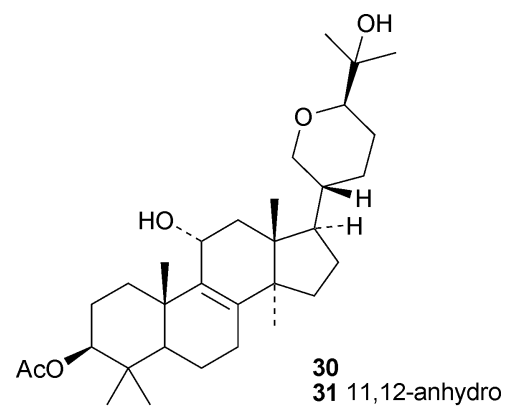

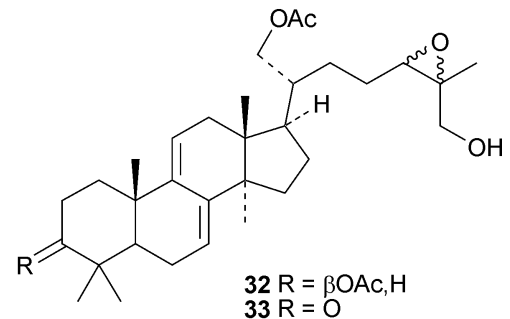<smiles>[R]C(=O)[R]([Y9])([H])[H]</smiles>

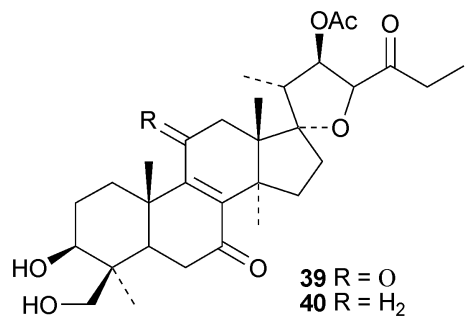

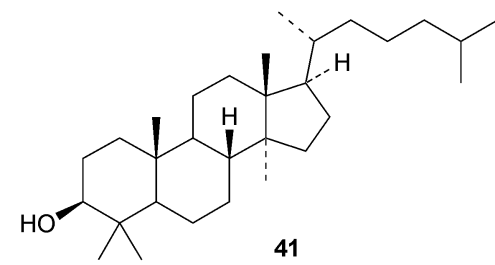<smiles>C=C(C)CCC[C@]1(C)OC(=O)[C@]23CC[C@]4(C)C(=CCC5C(C)(C)[C@@H](O)CC[C@]54C)[C@@]2(C)CCC13C</smiles> 
Erylosides $\mathrm{R}_{1}, \mathrm{~T}_{1}, \mathrm{~T}_{2}, \mathrm{~T}_{3}, \mathrm{~T}_{4}, \mathrm{~T}_{5}$ and $\mathrm{T}_{6}$ are lanostane saponins with known genins from the Caribbean sponge Erylus formosus. ${ }^{41}$ Of the five new saponins, scillanostasides A-E, isolated from the bulbs of Scilla scilloides, only A and B have new genins 39 and $40{ }^{42}$ Lanostan-3 $\beta$-ol 41 is a new genin of a diglucuronoside from the flowers of Punica granatum. ${ }^{43}$
Cucumariosides $\mathrm{H}_{5}, \mathrm{H}_{6}, \mathrm{H}_{7}$ and $\mathrm{H}_{8}$ are new holostane glycosides from the sea cucumber Eupentacta fraudatrix. ${ }^{\mathbf{4 4}}$ Cucumarioside $\mathrm{H}_{8}$ has a new genin 42 with an unusual 16,22epoxide. Patagonicosides $\mathrm{B}$ and $\mathrm{C}$, sulphated glycosides from the sea cucumber Psolus patagonicus, display antifungal activity. ${ }^{45}$ Patagonicoside $B$ has the new genin 43. Two new

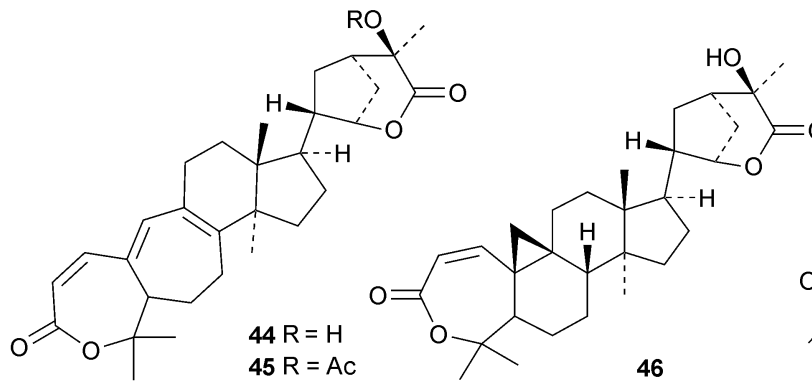

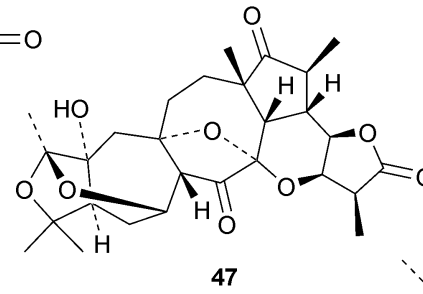

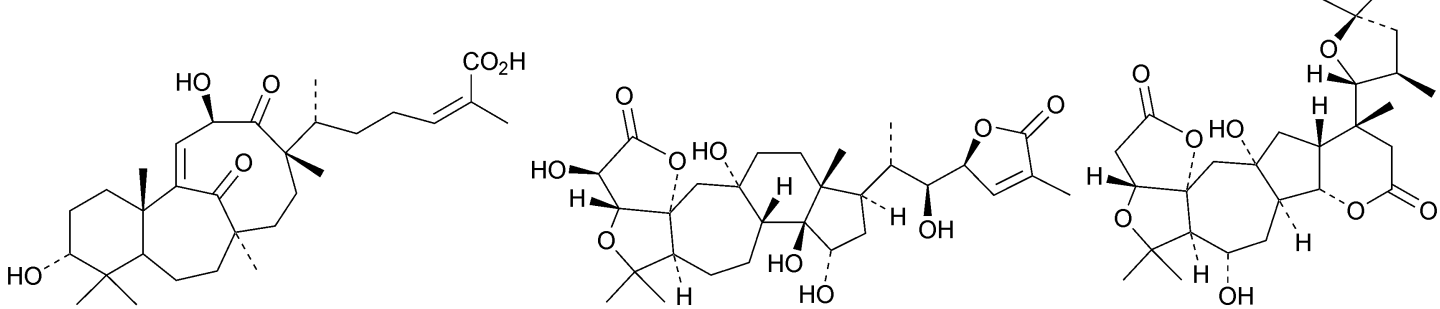

48

49

50

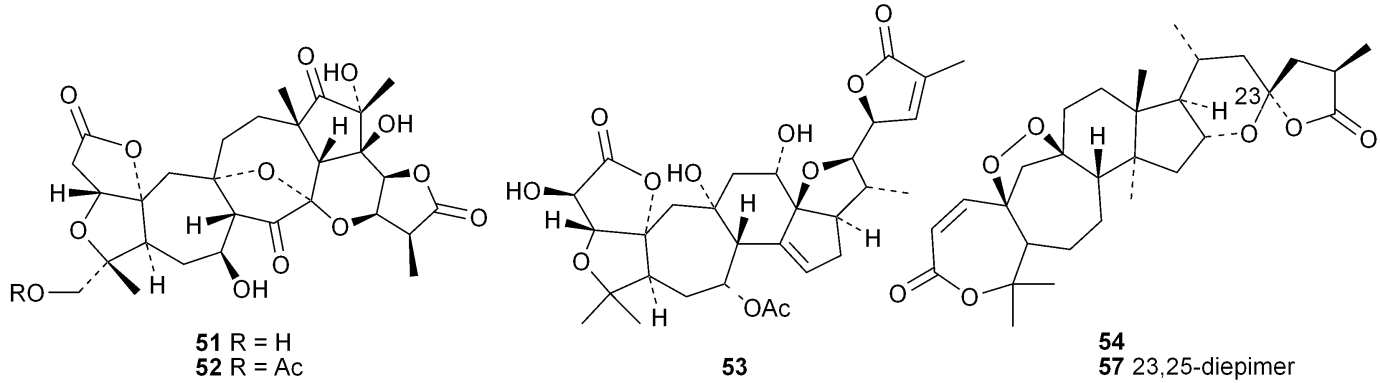

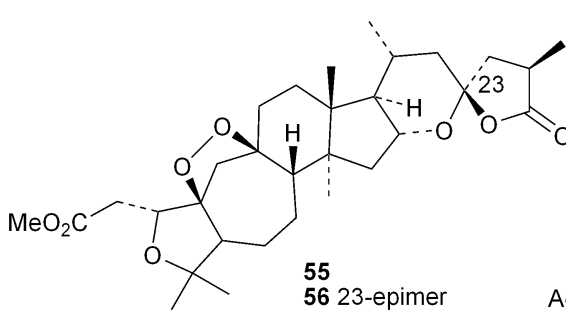

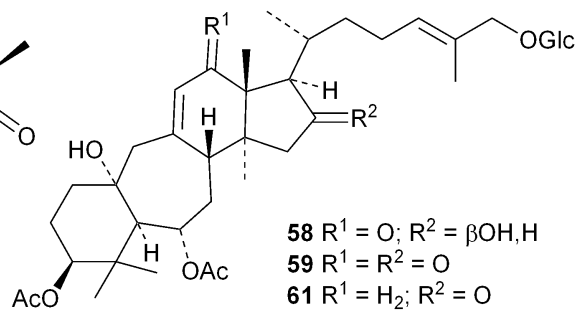

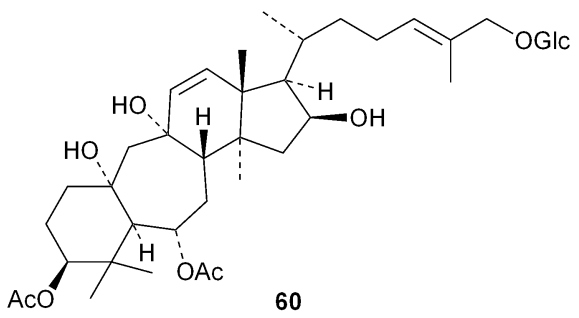<smiles>[R10]O[C@H]1CCC2=CC3=CC[C@]4(C)[C@@H]([C@H](C)CC(=O)[C@@H]5OC5(C)C)C(=O)C[C@]4(C)C3=CC[C@H]2C1(C)C</smiles><smiles>C/C(=C\[C@@H](O)[C@@H](O)[C@@H](C)[C@H]1CC[C@H]2[C@@H]3CCC4=C(CC[C@H](O)C4(C)C)CC3=CC[C@@]21C)C(=O)O</smiles> 
glycosides with known genins, liouvillosides $\mathrm{A}_{4}$ and $\mathrm{A}_{5}$, have been isolated from the sea cucumber Staurocucumis liouvillei. ${ }^{\mathbf{4 6}}$

Interesting new compounds from Schisandra species include henrischinins A-C 44-46 from Schisandra henryi with an oxabicyclo[3.2.1] octane moiety in the side chain, ${ }^{47}$ the bisnorderivative schinarisanlactone A $\mathbf{4 7}$ from Schisandra arisanensis ${ }^{\mathbf{4 8}}$ and the tricyclic derivative schiglautone A $\mathbf{4 8}$ from the stems of Schisandra glaucescens. ${ }^{49}$ The structure of henrischinin B 45 was confirmed by X-ray analysis. $2 \beta$-Hydroxymicrandilactone $\mathrm{C} \mathrm{49,50}$ schintrilactone C $\mathbf{5 0}^{51}$ and wilsoniadilactones D-F $\mathbf{5 1 - 5 3 ^ { 5 2 }}$ are new constituents of Schisandra chinensis, Schisandra sphenanthera and Schisandra wilsoniana, respectively. Four new peroxylactones, pseudodarolides $\mathrm{Q}_{2} 54, \mathrm{~T}_{1} 55$ and $\mathrm{T}_{2} 56^{53}$ and 25-epipseudolarolide $\mathrm{Q} \mathbf{5 7}{ }^{\mathbf{5 4}}$ have been isolated from Pseudolarix kaempferi. Huangqiyenins G-J 58-61 are new saponins from Astragalus membranaceus. ${ }^{55}$ The xyloside cimipodocarpaside 62 has been reported from Cimifuga racemosa. ${ }^{56}$ The myxomycete Tubulifera arachnoidea afforded the new 9,10-secocycloartane tubiferic acid $63 .{ }^{57}$
Sinocalycanchinensins A-H 64-71 are 29-norcycloartanes from the leaves of Sinocalycanthus chinensis. ${ }^{58}$ Sinocalycanchinensins A-E 64-68 are 3,4-seco-derivatives while sinocalycanchinensin F 69 has a 2,3-cleaved ring A. Other 3,4-cleaved cycloartanes include gardenoins I 72 and $\mathrm{J} 73$ from the exudates of Gardenia thailandica ${ }^{59}$ and coccinetanes B-G 74-79 from Kadsura coccinea. ${ }^{60}$ Secopisonic acid from Pisonia umbellifera $^{6 \mathbf{1}}$ and gardenoin $\mathrm{H}$ from the apical buds of Gardenia obtusifolia ${ }^{62}$ are identical with coccinetane E 77. Gardenoins E-G 80-82 are other constituents of Gardenia obtusifolia. ${ }^{\mathbf{6 2}}$ Angustific acid A 83, from Kadsura angustifolia, has an unusual bridged lactone. ${ }^{63}$ It is accompanied by angustific acid B $\mathbf{8 4}$ and angustifodilactones A 85 and B 86. The compounds are reported to have anti-HIV activity.

In separate studies ten new cycloartanes and glycosides 87$\mathbf{9 6} \mathbf{6}^{\mathbf{6 4}}$ and three new glycosides, two (97 and 98) with new genins, ${ }^{65}$ have been reported from Cimifuga foetida. Six new glycosides 99-104 have been isolated from the rhizome of Cimifuga heracleifolia ${ }^{66}$ and two, tareciliosides $\mathrm{L}$ and $\mathrm{M}$ with new genins<smiles></smiles>

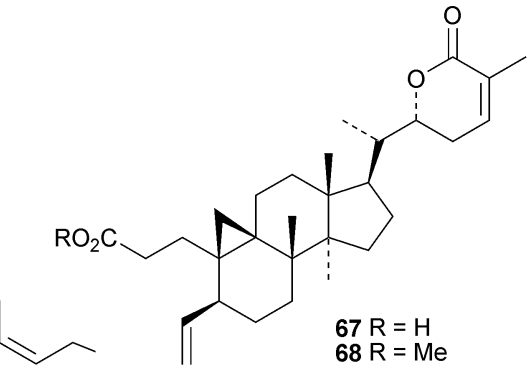

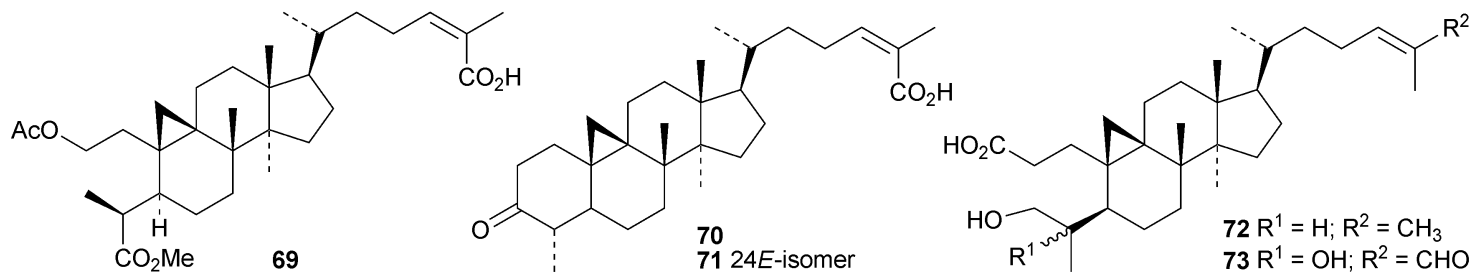

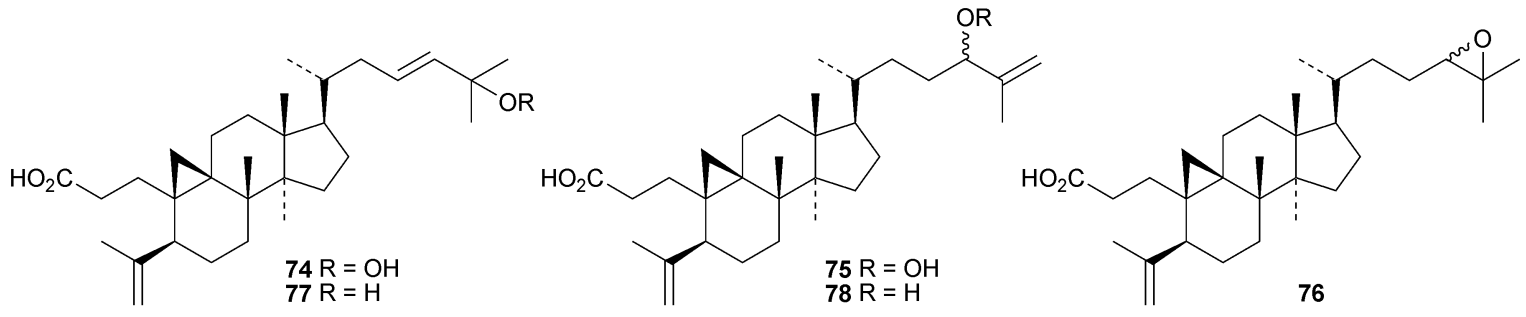

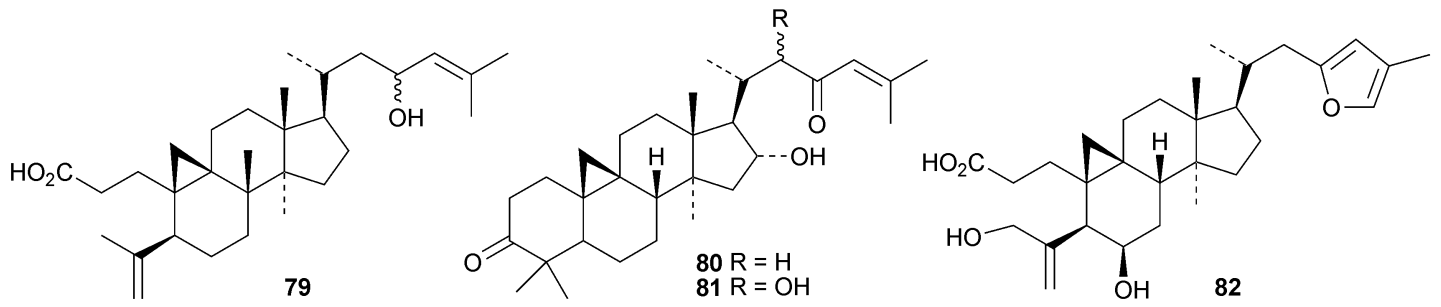




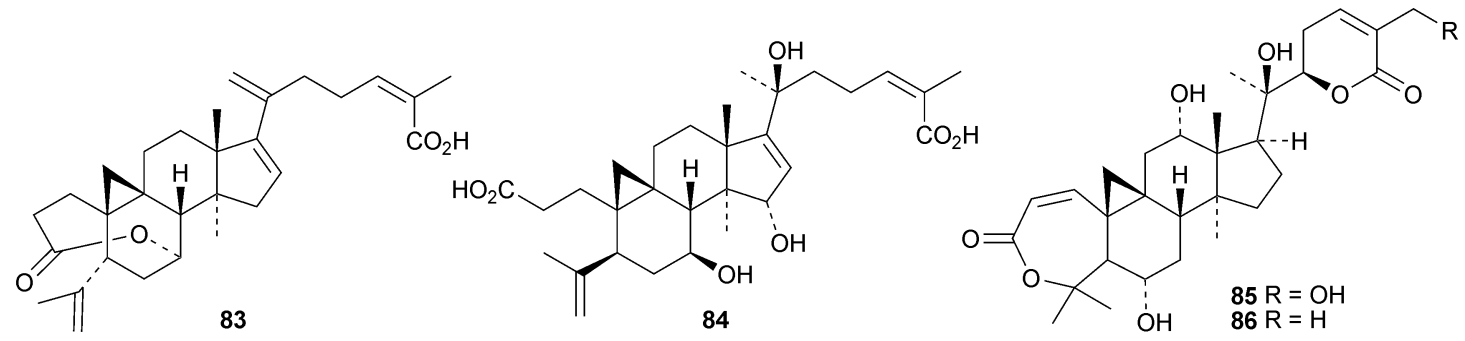

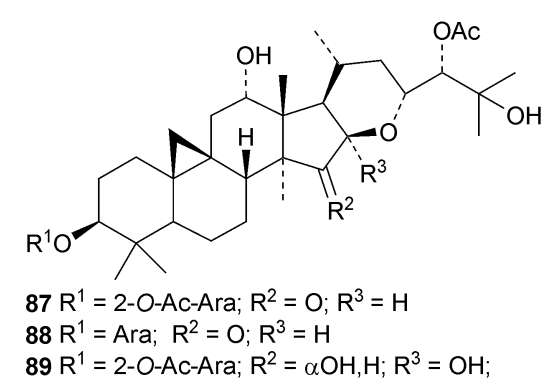

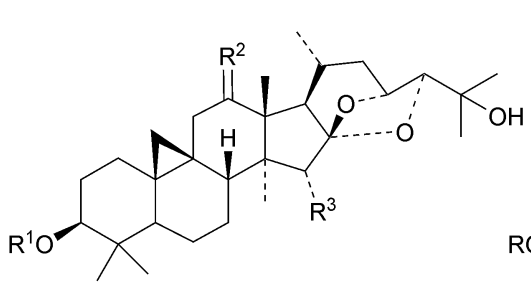

$90 \mathrm{R}^{1}=\mathrm{H} ; \mathrm{R}^{2}=\beta \mathrm{OH}, \mathrm{H} ; \mathrm{R}^{3}=\mathrm{OH} ; 25,26$-anhydro $91 R^{1}=$ Ara $R^{2}=H_{2} ; R^{3}=O H ; 25,26$-anhydro $92 \mathrm{R}^{1}=\mathrm{H} ; \mathrm{R}^{2}=\mathrm{O} ; \mathrm{R}^{3}=\mathrm{OH}$ $93 \mathrm{R}^{1}=\mathrm{H} ; \mathrm{R}^{2}=\beta \mathrm{OH}, \mathrm{H} ; \mathrm{R}^{3}=\mathrm{H}$ $94 \mathrm{R}^{1}=2$-O-Ac-Ara $\mathrm{R}^{2}=\mathrm{H}_{2} ; \mathrm{R}^{3}=\mathrm{OH} ; 24 R$ $95 \mathrm{R}^{1}=2-\mathrm{O}-\mathrm{Ac}-\mathrm{Xyl} ; \mathrm{R}^{2}=\mathrm{H}_{2} ; \mathrm{R}^{3}=\mathrm{OH}$

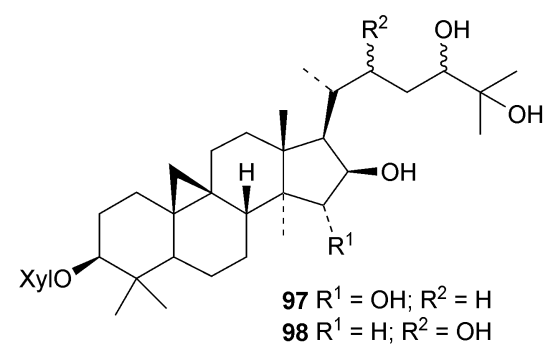

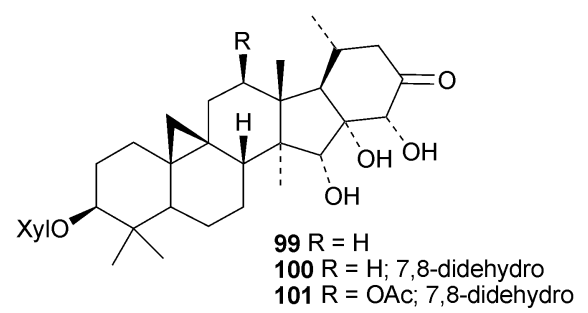

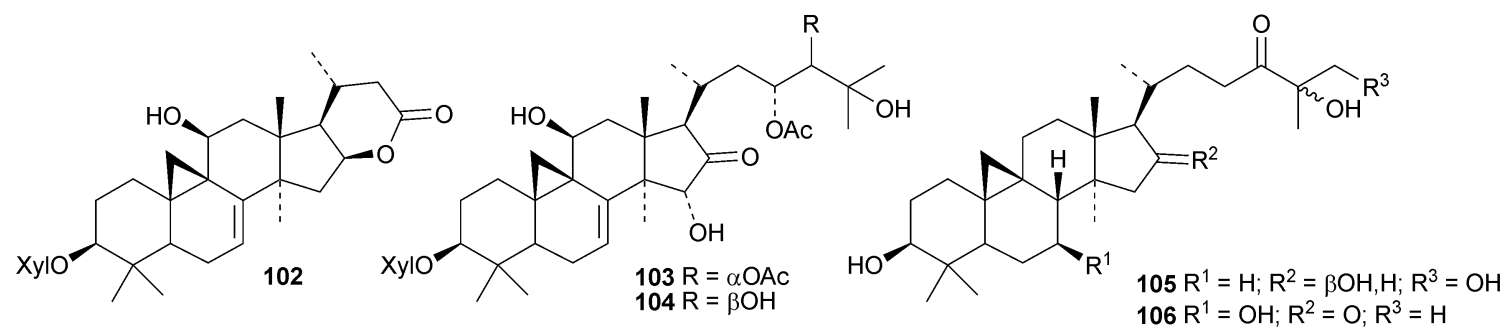

105 and 106, from the leaves of Tarenna gracilipes ${ }^{67}$ Tareciliosides $\mathrm{H}-\mathrm{K}$ have known genins.

The 18(13 $\rightarrow$ 17)-abeocycloartane $\mathbf{1 0 7}$ is a constituent of the bark and leaves of Garcinia benthami, where it occurs along with the 14,17-friedolanostanes 108-110. ${ }^{68}$ Other new cycloartanes include combretanones A-G 111-117 and combretic acids A 118 and B 119 from Combretum quadlangulare, ${ }^{69}$ bicusposides D-F 120-122 from Astragalus bicuspis, ${ }^{70}$ macrostachyosides A 123 and B 124 from Mallotus macrostachyus, ${ }^{71}$ cycloart-24-ene-2 $\alpha, 3 \beta$ diol 125 from the stigma of Zea mays ${ }^{72}$ and boniatic acids A 126 and B 127 from Radermachera boniana..$^{73}$ Bonianic acids A 126 and B 127 showed some antitubercular activity. Codonopilates A-C 128-130 are cycloartane esters from Codonopsis pilosula. ${ }^{74}$
Novel cycloartane saponins with known genins include askendoside $\mathrm{K}$ from Astragalus taschkendicus, ${ }^{75}$ cicerosides A and B from Astragalus cicer ${ }^{76}$ shengmaxinsides $\mathrm{A}-\mathrm{C}$ from Cimicifuga simplex, ${ }^{77}$ and unnamed saponins from Astragalus mucidus. $^{78}$ The biological activities of cycloartane triterpenoids have been reviewed..$^{9}$

Machilusides A 131 and B 132, from the stem bark of Machilus yaoshansis, are cucurbitane glycosides with an unusual $C$-glycoside moiety. ${ }^{80}$ The roots of Machilus yaoshansis afforded seven new glycosides 133-139. ${ }^{81}$ These authors also revised the C-24 configurations of several known compounds, including cucurbitacins S and T and colocyhthins A, B and C, from $24 S$ to 24R. Compounds 140 and 141, from the roots of Wilbrandia 


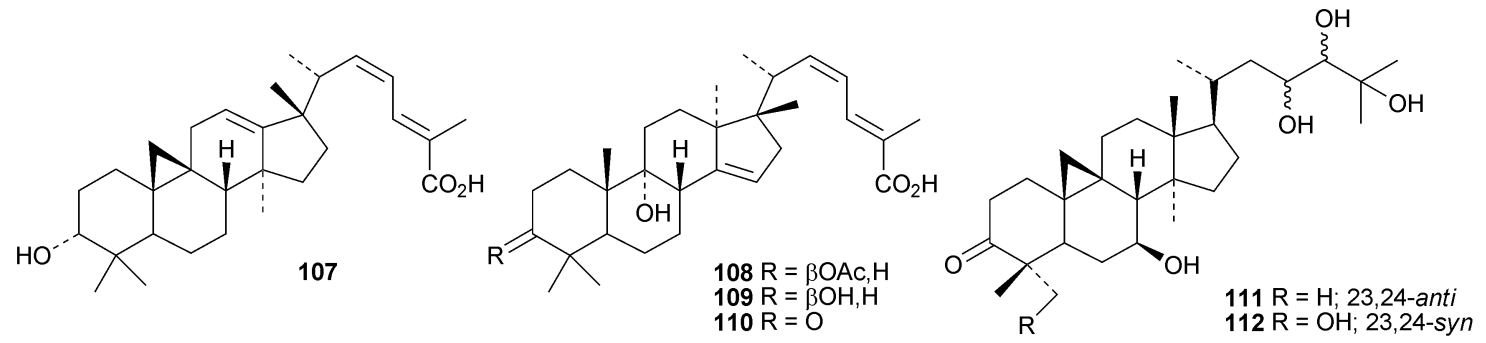

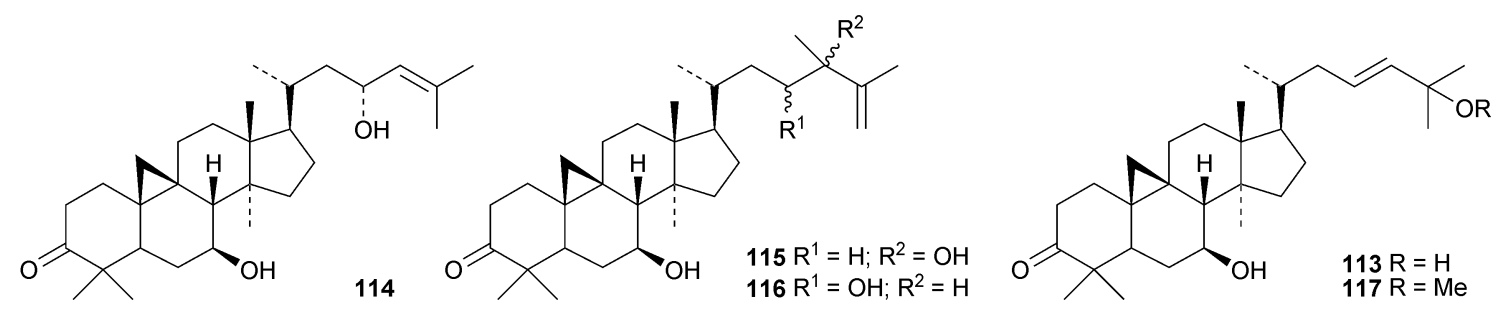

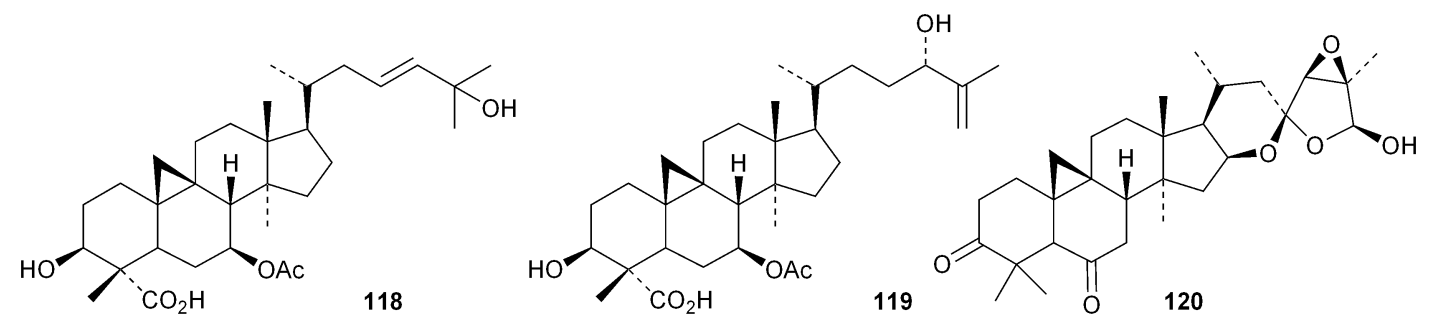

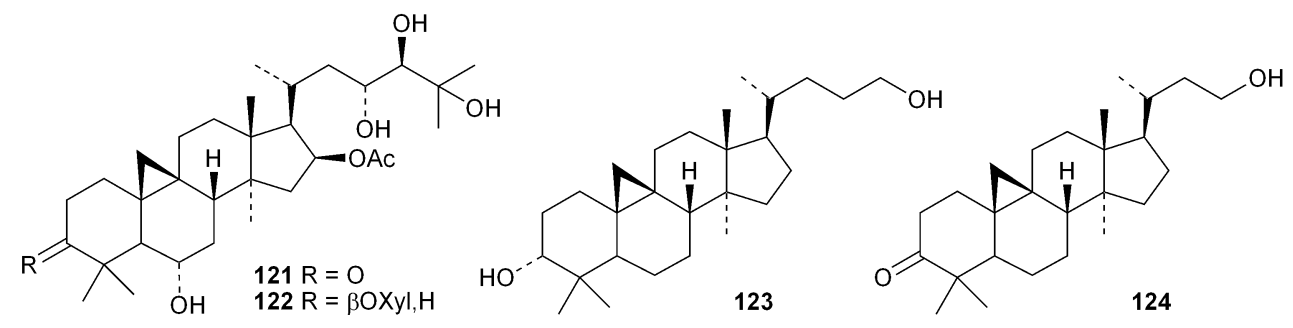

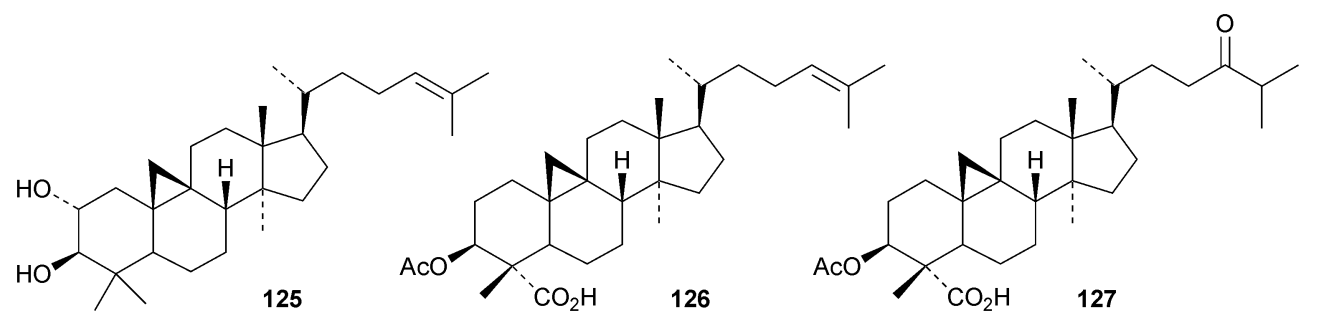

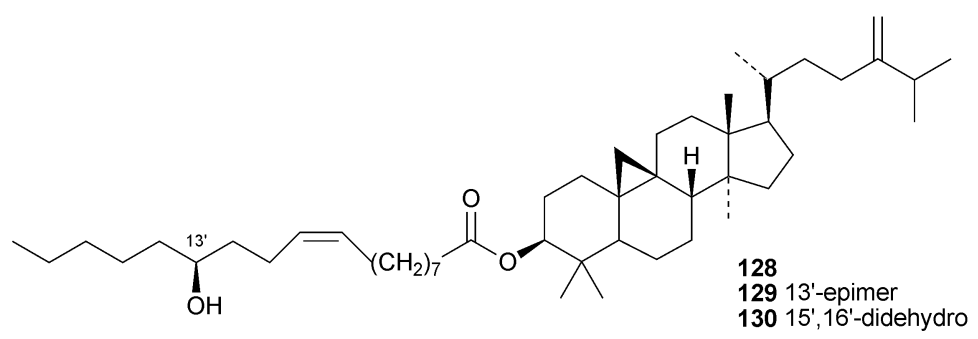

ebracteata, are reported to have cytotoxic activity. ${ }^{82}$ New cucurbitanes from Momordica charantia include the antioxidants taiwacins A 142 and B 143 from the stems and fruit, ${ }^{83}$ 144-148 ${ }^{84}$ and 149 and $150 .{ }^{85}$ Compound 148 is a 19-nor-derivative with an aromatic ring $\mathrm{B}$. The biological activities of compounds from Momordica charantia have been reviewed. ${ }^{\mathbf{8 6}}$ 
<smiles>[Y19][R](=O)O</smiles>

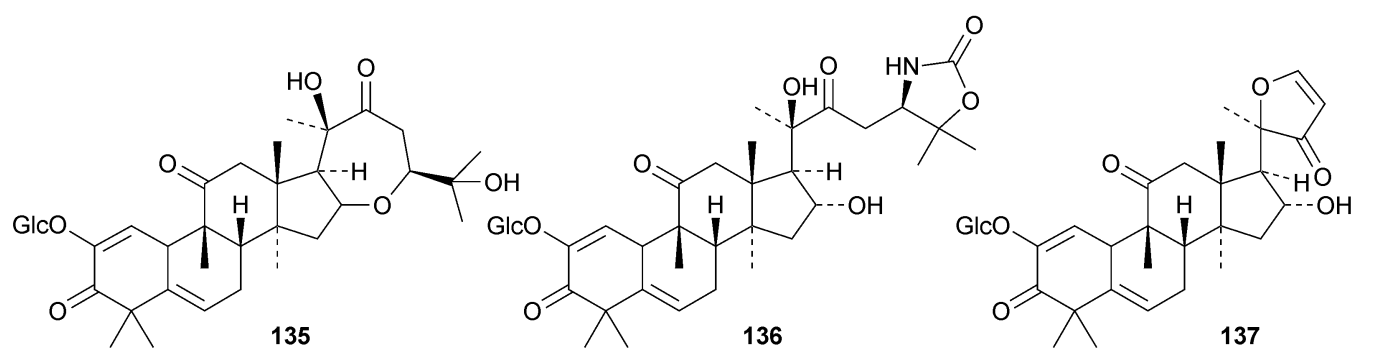

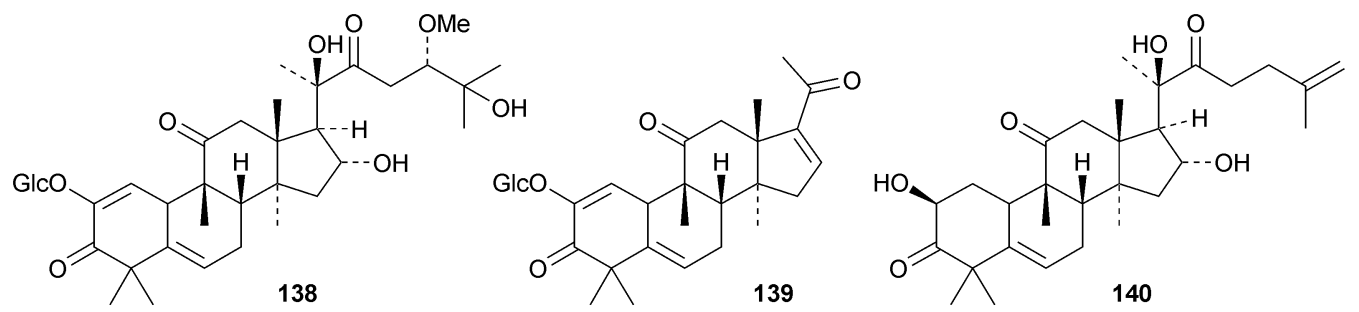

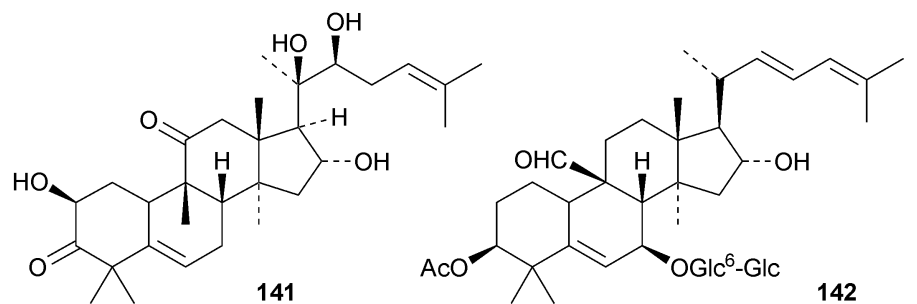<smiles>CC(C(=O)O)[C@H]1CC[C@H]2C(=O)C=C3C(CCC(=O)C3(C)C)[C@@]2(C)CCC1(C)C</smiles>

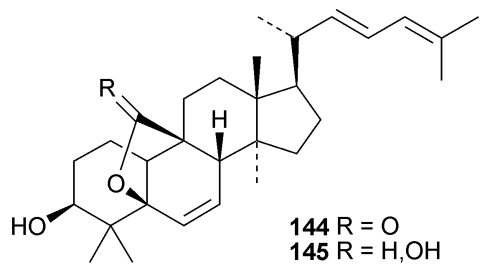<smiles>[R][R]1ccco1</smiles><smiles>CC(C)=C/C=C\C(C)[C@H]1CC[C@]2(C)c3ccc4c(c3CC[C@]12C)CC[C@H](O)C4(C)C</smiles>

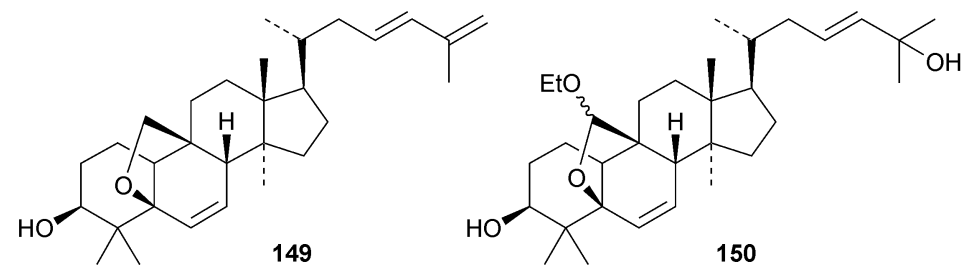

\section{The dammarane group}

Gypensapogenins A 151 and B 152 are modified dammaranes, with an unusual ring A, from Gynostemma pentaphyllum, where they are found with gypensapogenins C 153, D 154 and the glucoside $155^{87}$ and the 21,24-cyclo derivative 156 and the nonanordammarane $157 .{ }^{88}$ The structure of gypensaponin A 151 was confirmed by X-ray analysis. Other new dammaranes 
include gardaubryones A-C 158-160 from Gardenia aubryi, ${ }^{89}$ 161-163 from the berries of Panax ginseng, ${ }^{90}$ 164-170 from the floral spikes of Betula platyphylla var. japonica, ${ }^{91}$ the 24 -epimers 171 and 172 from the apical buds of Gardenia collinsae, ${ }^{92}$ dammara-20(22),24-diene-3 $\beta, 26,27-$ triol 173 from the leaves and twigs of Rhus taitensis ${ }^{25}$ and the $\alpha$-ketol 174 from the exudate of the leaves of Cerasus yedoensis. ${ }^{93}$ The structure recently proposed for ailexcelone, from Ailanthus excelsa, is similar to that of gardaubryone B 159 but its spectroscopic data are inconsistent with this structure, The revised structure, 24,25-dihydroxytirucall-7-en-3-one, has been proposed and the structure of the corresponding $3 \beta$-hydroxy-derivative should also be revised. ${ }^{\mathbf{9}}$
Four new saponins, operculinosides A-D 175-178, have been reported from the aerial parts of Operculina turpethum. ${ }^{94}$ The structure of operculinoside A $\mathbf{1 7 5}$ was confirmed by X-ray analysis. Of the six saponins ginsenosides $\mathrm{Re}_{1}-\mathrm{Re}_{6}$ have been reported from the root of Panax ginseng, only ginsenoside $\mathrm{Re}_{5} 179$ has a new genin. ${ }^{95}$ Panajaponol, from the roots of Panax japonicus var. major, is identical to ginsenoside $\mathrm{Re}_{5} 179$ but was drawn with the wrong double bond geometry. ${ }^{96}$ Reviews on the pharmacological activities of the ginsenosides have appeared. ${ }^{97,98}$

Novel dammarane saponins with known genins include betalnosides B and C from Betula alnoides, ${ }^{99}$ centellosides A and $\mathrm{B}$ and ginsenosides Mc and Y from Centella asiatica, ${ }^{\mathbf{1 0 0}}$ ginsenosides $\mathrm{Ra}_{4}-\mathrm{Ra}_{9}{ }^{101}$ and $20 R$-ginsenoside $\mathrm{ST}_{2}{ }^{102}$ from Panax

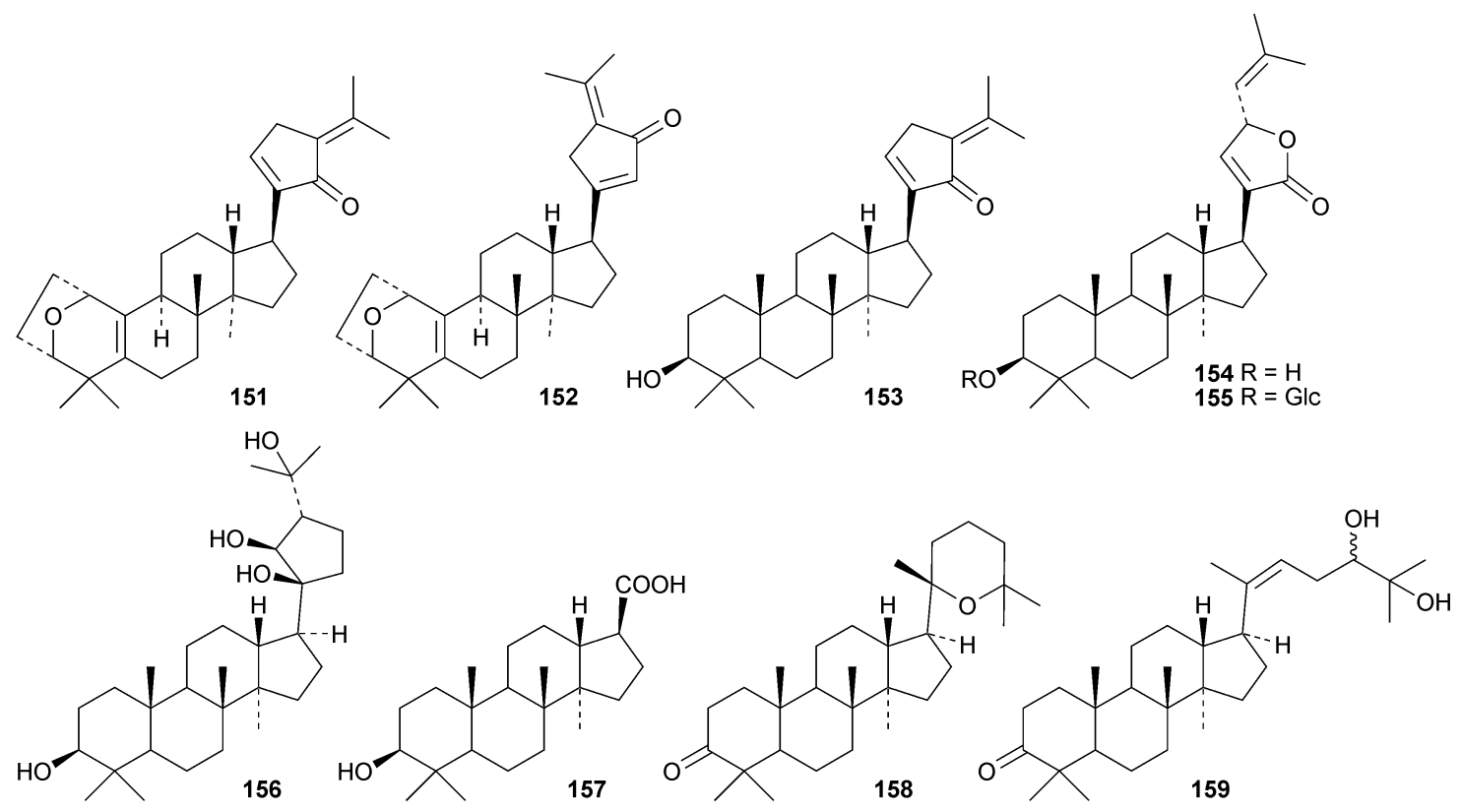

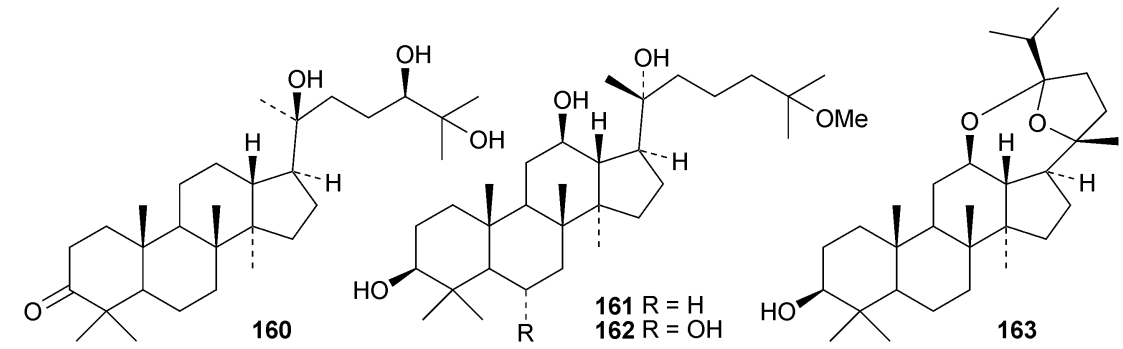

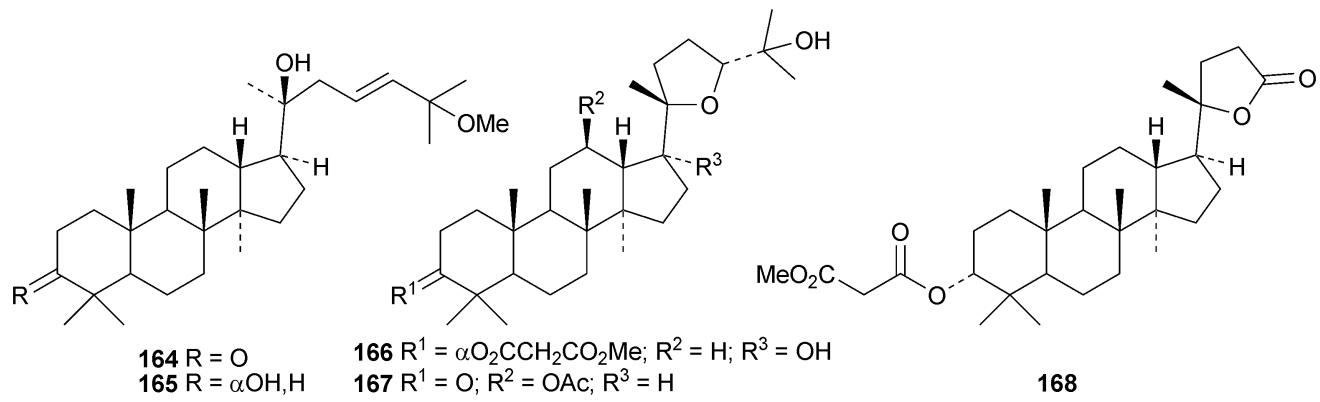




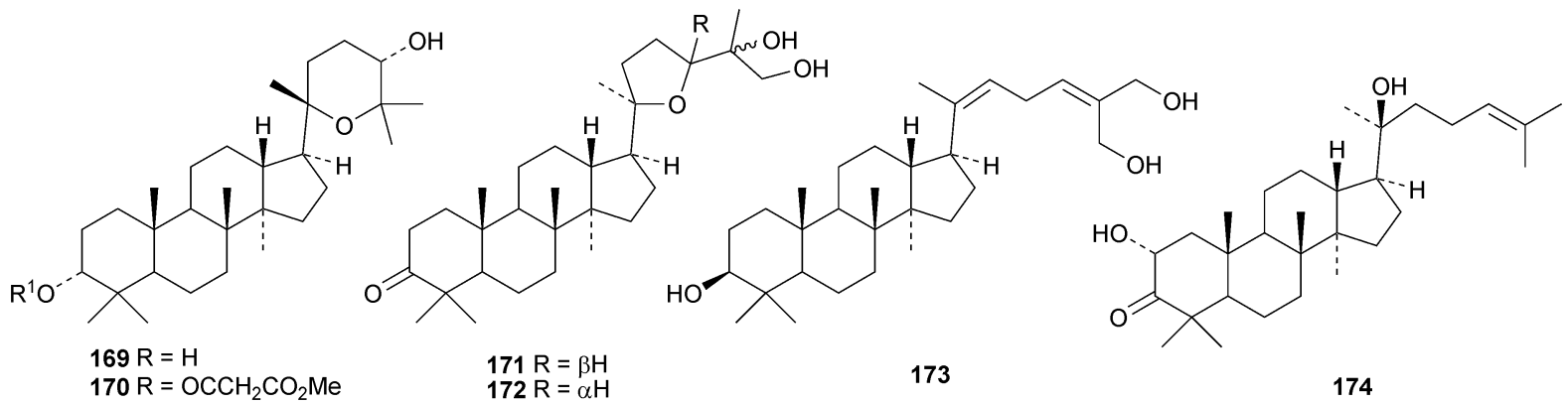

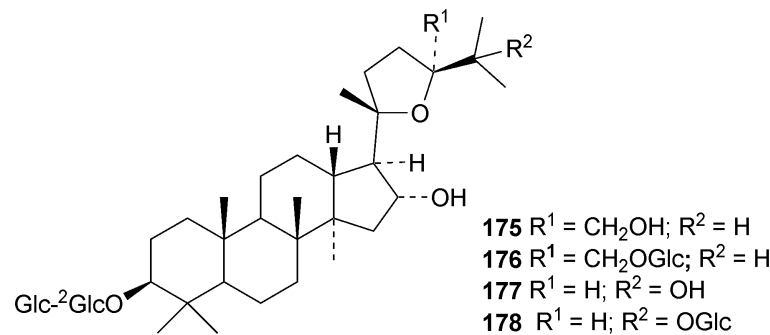

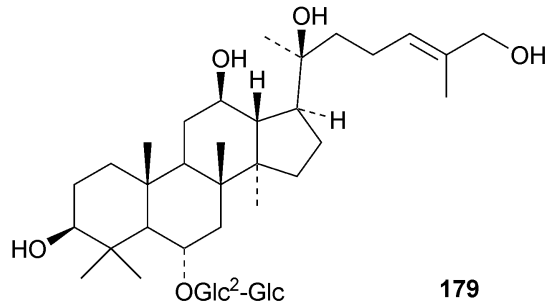

ginseng, gypenosides GC1-GC7 from Gynostemma pentaphyllum, ${ }^{\mathbf{1 0 3}}$ notoginsenosides $\mathrm{SFt}_{1}-\mathrm{SFt}_{4}$ from Panax notoginseng, ${ }^{104}$ pseudoginsenosides $\mathrm{G}_{1}$ and $\mathrm{G}_{2}$ from Panax quinquefolium, ${ }^{\mathbf{1 0 5}}$ yesanchinosides $\mathrm{R}_{1}$ and $\mathrm{R}_{2}$ from Panax japonicus $^{\mathbf{1 0 6}}$ and unnamed saponins from Gynostemma pentaphyllum..$^{\mathbf{1 0 7}}$

Toona ciliata var. pubescens is the source of the tirucallane derivatives toonapubesins A-G 180-186. ${ }^{\mathbf{1 0 8}}$ Toonapubesin G 186 has a rearranged side chain. The tirucallanes 187-192, together with dysoxylumstatins A-C 193-195, have been reported from Dysoxylum lukii. ${ }^{109}$ Dysoxylumstatin C 195 is an apotirucallane $\gamma$-lactone. Several nor-tirucallane derivatives 196-199 have been isolated from Aphanamixis grandifolia. ${ }^{\mathbf{1 1 0}}$ Compound 199 was also isolated as dysolenticin $\mathrm{G}$ from the twigs and leaves of Dysoxylum lenticellatum, a rich source of interesting tirucallane derivatives including dysolenticin A 200, with its rearranged side chain, and dysolenticins B-F 201-205 and H-J 206-208. ${ }^{111}$ The structures of 200, 202, 203, 205 and 207 were confirmed by X-ray analyses. Other new tirucallane derivatives from Aphanamixis grandifolia include aphagranins A-G 209-215 ${ }^{112}$ and compounds 216-220. ${ }^{113}$ Several of these compounds look suspiciously like artefacts of the extraction process. Cornus walteri is also a good source of new tirucallane derivatives. ${ }^{114}$ The constituents of this plant include cornusalterins A-L 221-232. Ailanthusaltenin A, from the stem bark of Ailanthus altissima, ${ }^{\mathbf{1 1 5}}$ is the same as cornusalterin D 224. Other new tirucallanes include 233 from Euphorbia sapinii, ${ }^{30}$ 234 from the resin of Boswellia carterii, ${ }^{116}$ the dihydroxy acid

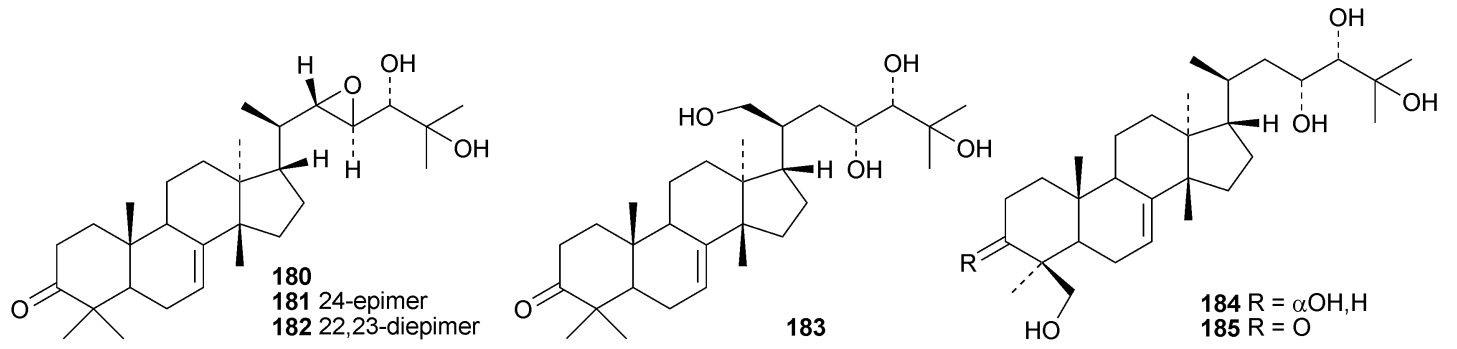<smiles>C[C@H](CC(O)C(C)(C)C=O)[C@H]1CC[C@]2(C)C3=CCC4C(C)(C)C(=O)CC[C@]4(C)C3CC[C@]12C</smiles>

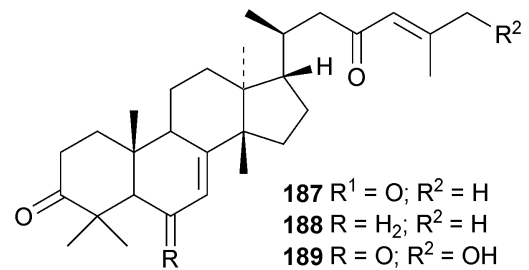<smiles>C[C@H](C/C=C/C(C)(C)O)[C@H]1CC[C@]2(C)C3=CCC4C(C)(C)[C@@H](O)CC[C@]4(C)C3CC[C@]12C</smiles> 


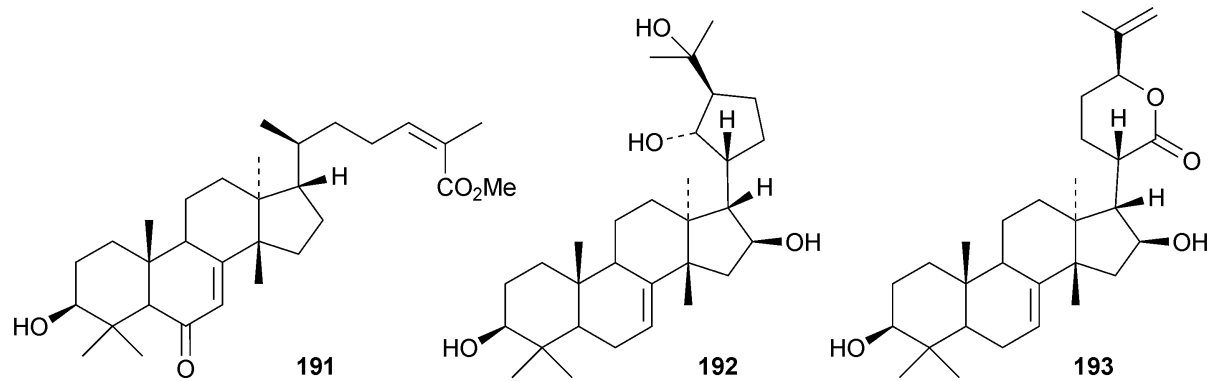<smiles>CC1(C)OC1CC(=O)C=C[C@H]1CC[C@]2(C)C3=CCC4C(C)(C)C(=O)CC[C@]4(C)[C@H]3CC[C@]12C</smiles><smiles>CC1(C)C(=O)CC[C@]2(C)C3CC[C@]4(C)[C@@H]([C@H]5COC(=O)C5)CC[C@]4(C)C3=CC[C@H]12</smiles>

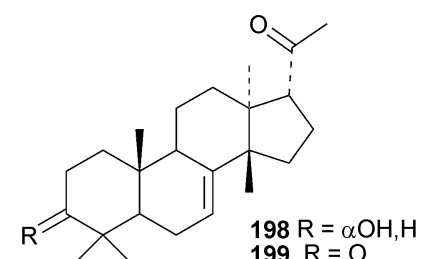<smiles>CC1(C)C2CC=C3C(CC[C@]4(C)C3CC[C@@]4(C)[C@H]3COC(=O)[C@](O)(C(C)(C)O)C3)[C@@]2(C)CC[C@@H]1O</smiles>

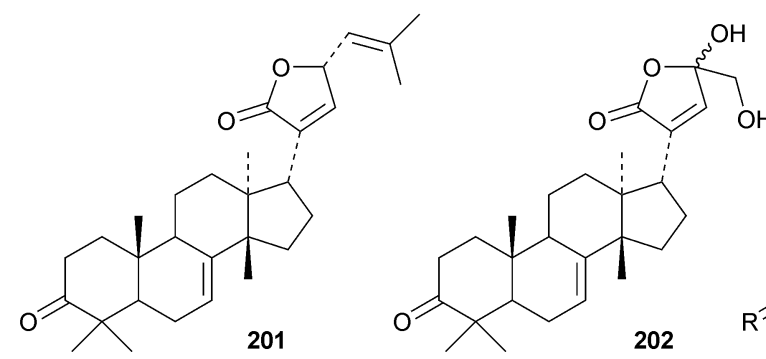

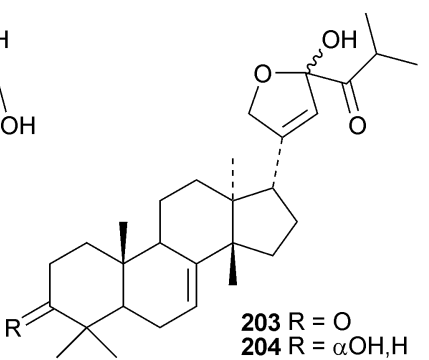<smiles>CCOC1(C)CC([C@H]2CC[C@]3(C)C4=CCC5C(C)(C)C([OH2+])CC[C@]5(C)[C@H]4CC[C@]23C)CO1</smiles>

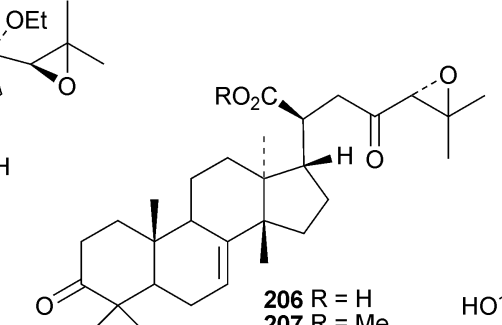<smiles>CC1(C)C(C2=CC(=O)NC2=O)CC[C@]2(C)C3CC[C@]4(C)NC(=O)C=CC4C3=CCC12</smiles>

235 from Jordanian propolis ${ }^{117}$ and 236 and 237 from Azadirachta indica. ${ }^{\mathbf{1 1 8}}$

Only seven euphane triterpenoids have been reported. They are compounds 238-241 from the bark of Broussonetia papyrifera ${ }^{119}$ nepetadiol 242 from Nepeta suavis ${ }^{\mathbf{1 2 0}}$ and the 21,24- cycloeuphane 243 and cinamodiol acetate 244 from the bark of Melia azedarach. ${ }^{\mathbf{1 2 1}}$

Cumingianols A-C 245-247 are cycloapotirucallane derivatives from Dysoxylum cumingianum. ${ }^{122}$ Other constituents include cumingianoside $\mathrm{R} \mathbf{2 4 8}$, a rare glycoside in this series, 

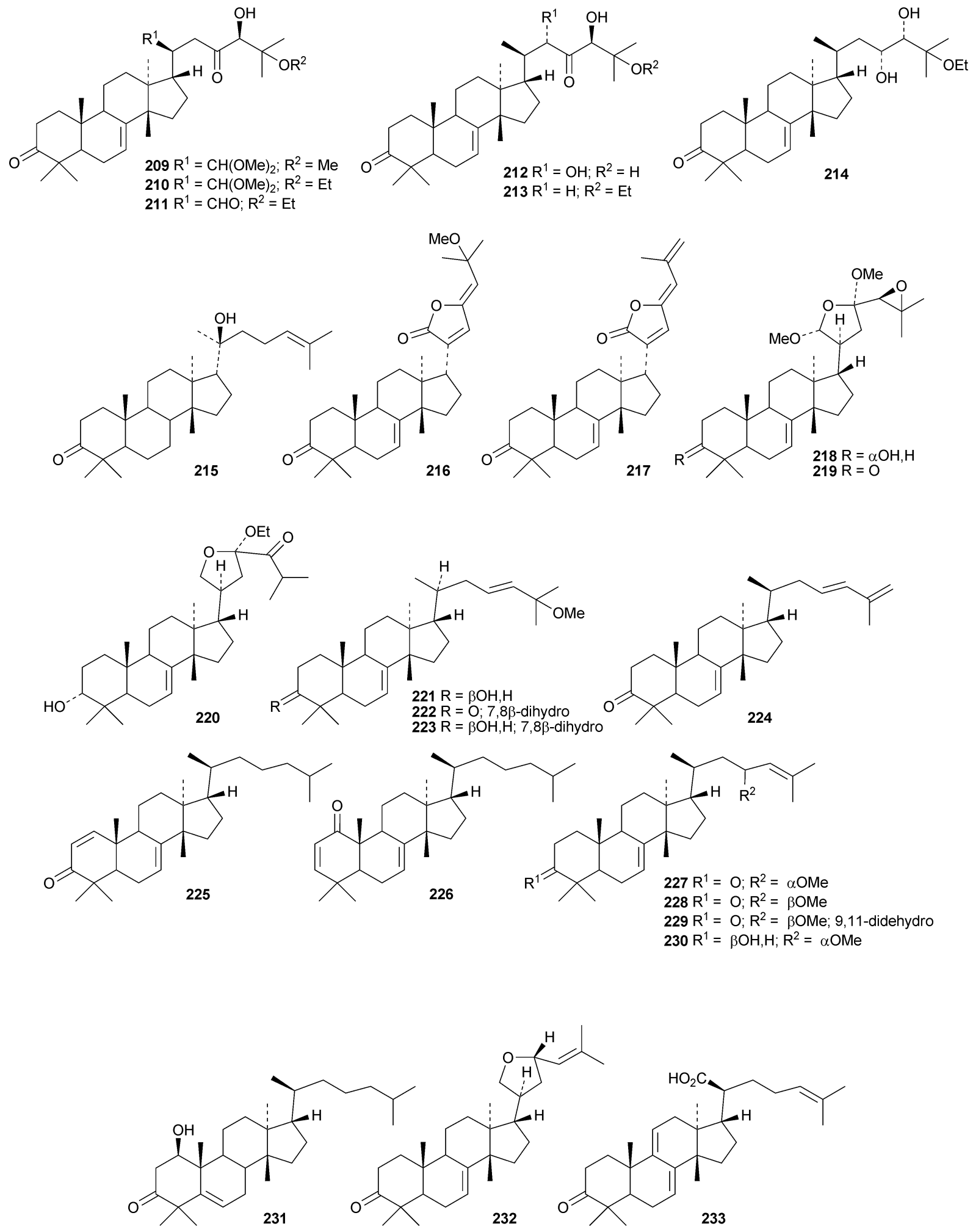

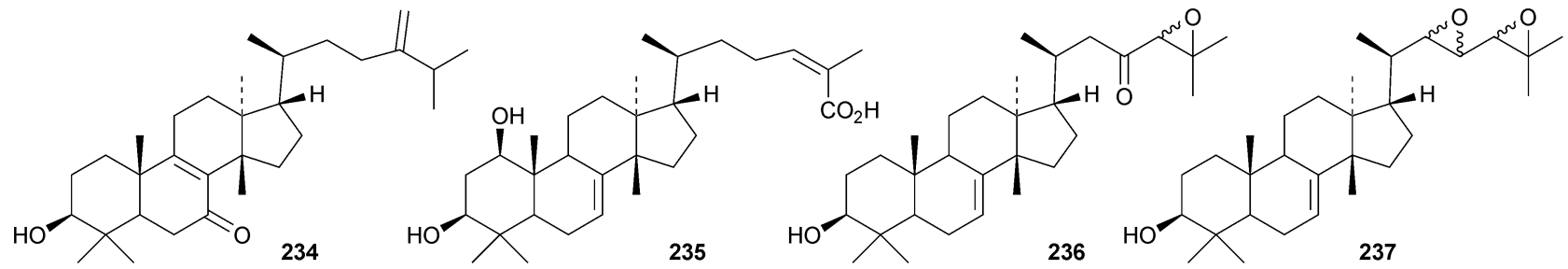




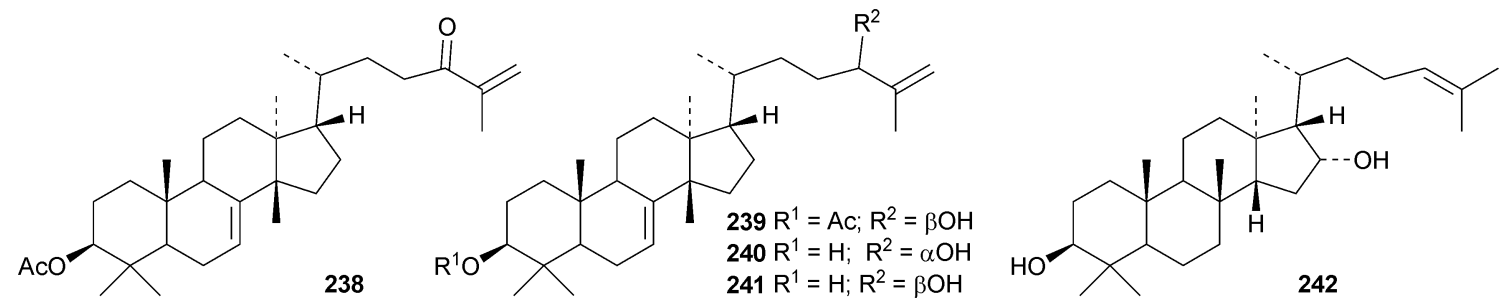

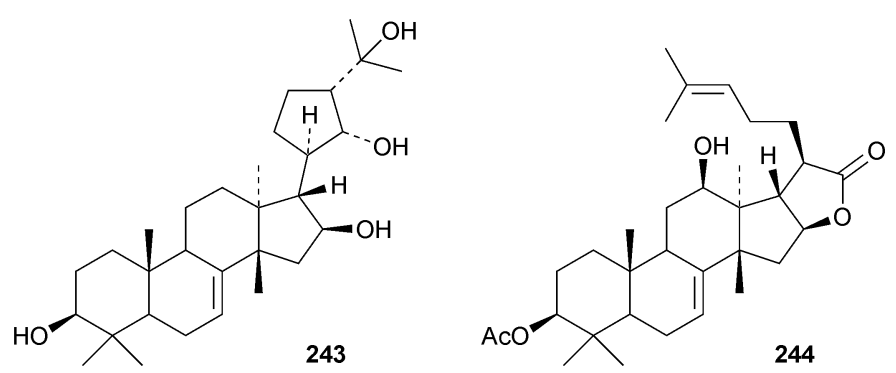

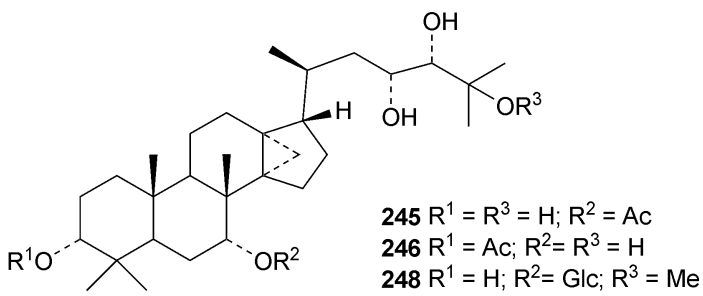

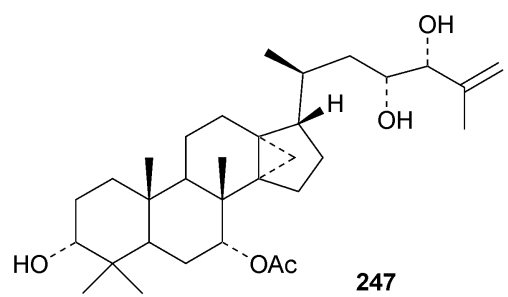

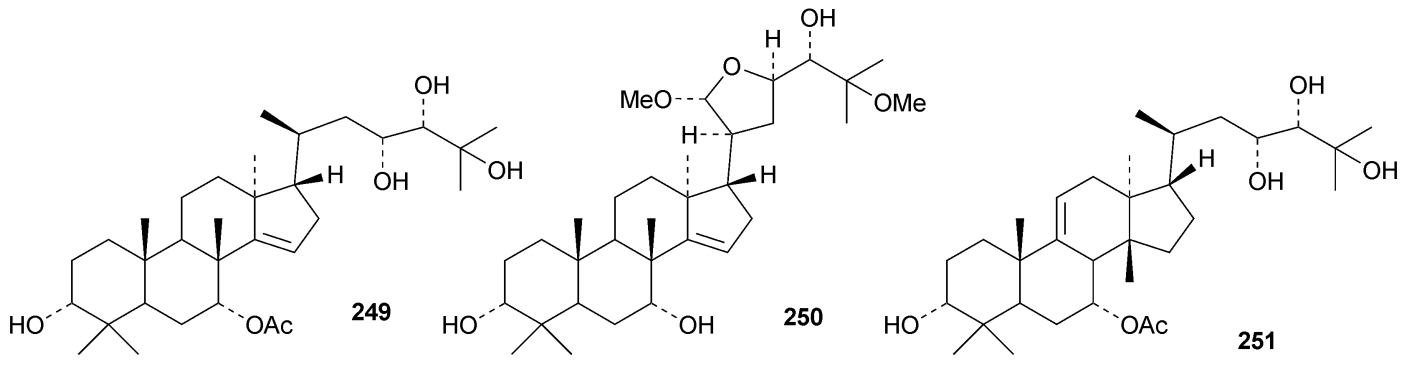

the apotirucallane derivatives, cumingianols D 249 and E 250, and the tirucallane, cumingianol F 251.

\subsection{Tetranortriterpenoids}

Reviews have appeared on limonoids from the Meliaceae ${ }^{123}$ and from Trichilia emetica ${ }^{124}$ and on the synthesis of limonoid natural products. ${ }^{125}$ Kokosanolides A 252 and C 253 are rearranged limonoids from the seeds and bark of Lansium domesticum cv. Kokossan. ${ }^{126}$ Other interesting derivatives include chisomicines A 254, B 255 and C 256 from the bark of Chisocheton ceramicus, ${ }^{127}$ 5,6-didehydrodesepoxyhaperforin C2 257 and harrpernoids B 258 and C 259 from the fruit of Harrisonia perforata, ${ }^{128}$ aphapolynins A 260 and B $261^{129}$ and aphanamolides A 262 and B $\mathbf{2 6 3}{ }^{\mathbf{1 3 0}}$ from Aphanamixis polystachya. The structures of kokosanolide A 252, chisomicines A-C 254-256 and aphapolyrin A 260 were all confirmed by X-ray analyses.
The lack of a furan ring is the notable feature of the tris-nor derivatives toonapubesic acids A 264 and B 265 from Toona ciliata var. pubescens. ${ }^{108}$ The structure of the methyl ester of toonapubesic acid A was confirmed by X-ray analysis. Ceramicines E-I 266-270 constitute a series of 1-oxo derivatives from Chisocheton ceramicus. ${ }^{131}$ The structure of the previously published ceramicine B $\mathbf{2 7 1}$ has been confirmed by X-ray analysis. Meliarachins A-K 272-282 are further limonoids from the twigs and leaves of Melia azedarach. ${ }^{132}$

Dasylactones A 283 and B 284 are degraded derivatives from Dictamnus dasycarpus. ${ }^{133}$ Raputiolide 285 is a ring-A cleaved limonoid from Raputia heptaphylla.$^{134}$ Toona ciliata var. henryi is a rich source of ring-B cleaved derivatives, affording toonacilianins A-L 286-297. ${ }^{135}$ Toonacilianins K 296 and L 297 are 29nor derivatives. Two further 29-nor derivatives, toonaciliatins $\mathrm{N}$ 298 and O 299 have been reported from Toona ciliata var. yunnanensis, where they occur along with toonaciliatin $\mathrm{P} \mathbf{3 0 0 .}{ }^{136}$ 


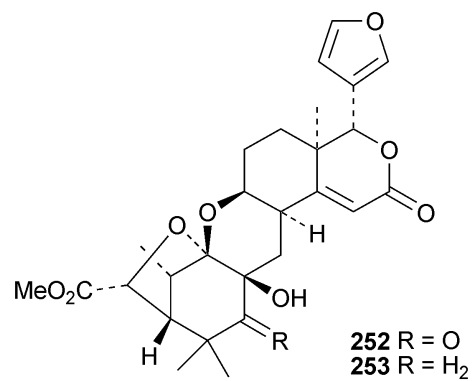

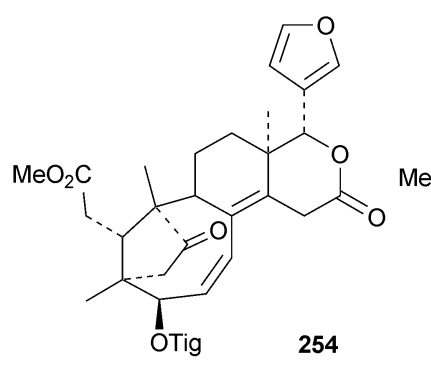

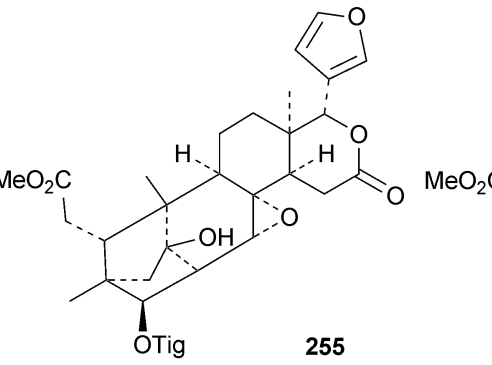

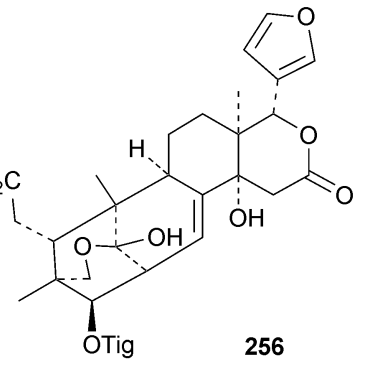<smiles>CC1(C)OC(=O)C=C[C@]2(C)C1=CC(=O)C=C1[C@@H]2CC[C@]2(C)[C@H](c3ccoc3)OC(=O)[C@]12C</smiles><smiles>CC12CC[C@@H]3O[C@@]4(CC3=C1C(=O)O[C@@H]2c1ccoc1)C[C@@H](C1(C)C=CC(=O)O1)C(C)(C)O4</smiles>

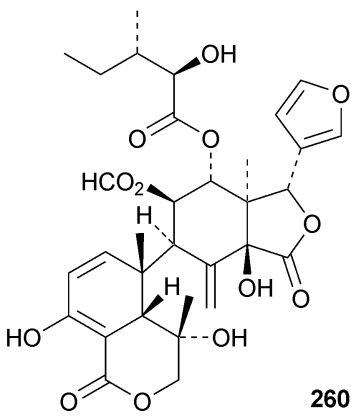

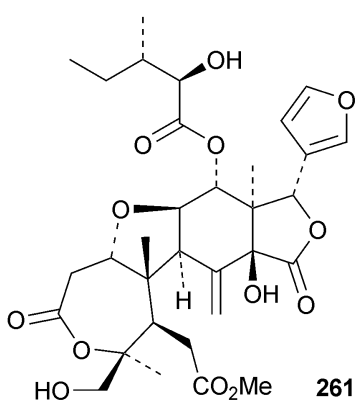

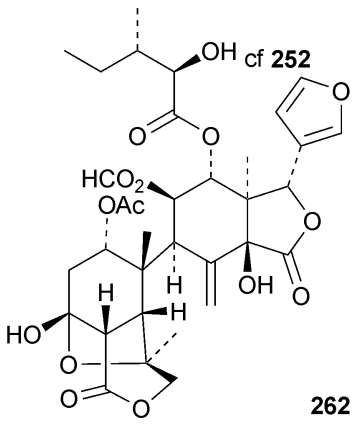

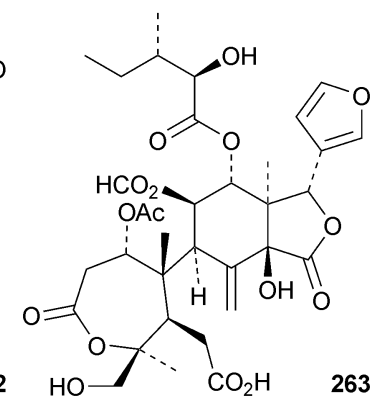

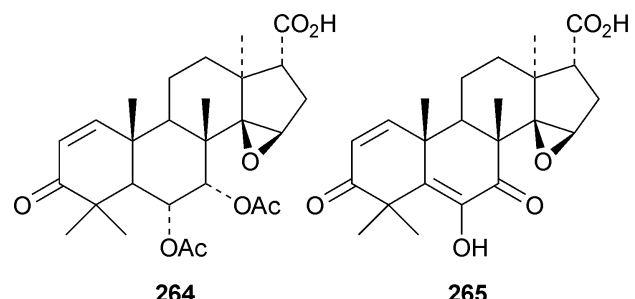

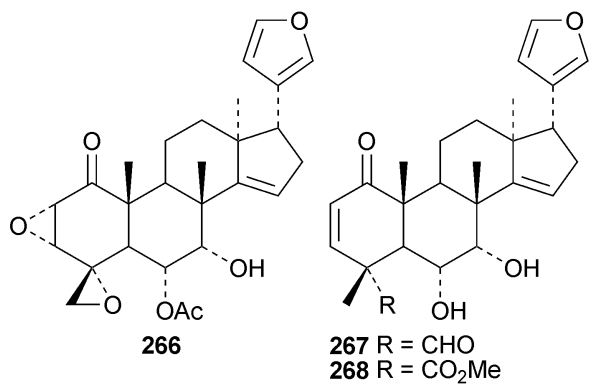

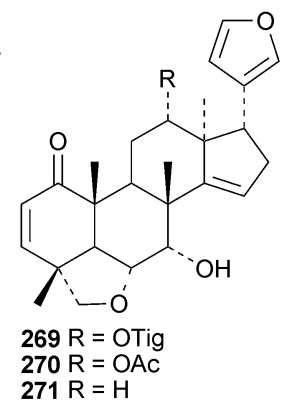

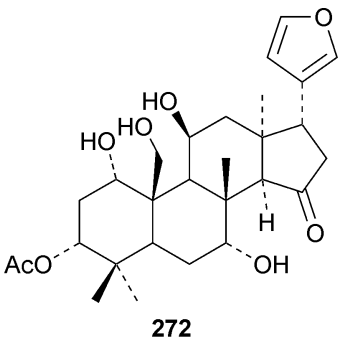

Three methyl angolensate derivatives 301-303 have been found in the root bark of Entandrophragma angolense, where they occur with the gedunin derivatives 304 and $305 .{ }^{137}$ Compound 301 is the same as moluccensin $\mathrm{O}$ which was published in 2010.
Thaimoluccensin A $\mathbf{3 0 6}$ is an andirobin derivative from the seeds of Xylocarpus moluccensis. ${ }^{\mathbf{1 3 8}}$ Although its structure was confirmed by X-ray analysis the wrong relative configuration was published in the original paper. 


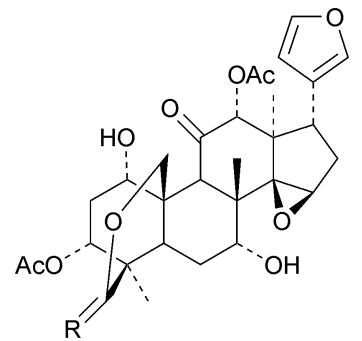

$273 \mathrm{R}=0$ $274 \mathrm{R}=\mathrm{OMe}, \mathrm{H}$

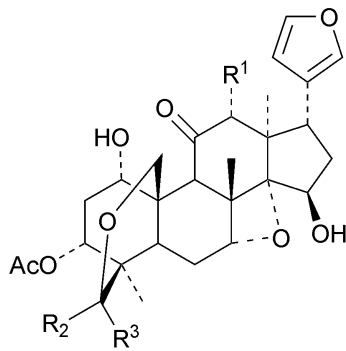

$275 R^{1}=O A c ; R^{2}=O M e ; R^{3}=H$ $276 \mathrm{R}^{1}=\mathrm{R}^{3}=\mathrm{OH} ; \mathrm{R}^{2}=\mathrm{H}$ $277 R^{1}=R^{2}=O H ; R^{3}=H$

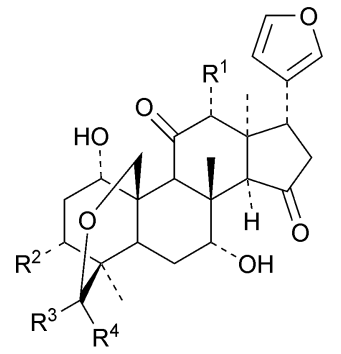

$278 \mathrm{R}^{1}=\mathrm{R}^{2}=\mathrm{OAC} ; \mathrm{R}^{3}=\mathrm{OMe} ; \mathrm{R}^{4}=\mathrm{H}$

$279 \mathrm{R}^{1}=\mathrm{OAc} ; \mathrm{R}^{2}=\mathrm{OH} ; \mathrm{R}^{3}=\mathrm{OMe} ; \mathrm{R}^{4}=\mathrm{H}$

$280 \mathrm{R}^{1}=\mathrm{R}^{2}=\mathrm{OH} ; \mathrm{R}^{3}=\mathrm{OMe} ; \mathrm{R}^{4}=\mathrm{H}$

$281 R^{1}=O H ; R^{2}=O A C ; R^{3}=H ; R^{4}=O M e$

$282 \mathrm{R}^{1}=\mathrm{R}^{2}=\mathrm{OAC} ; \mathrm{R}^{3}=\mathrm{H} ; \mathrm{R}^{4}=\mathrm{OMe}$<smiles>[R]c1cc(C2OC(=O)C3=C(C)CCCC32)c([R])o1</smiles>

$283 \mathrm{R}^{1}=\mathrm{O} ; \mathrm{R}^{2}=\mathrm{OH}, \mathrm{H}$ $284 \mathrm{R}^{1}=\mathrm{OH}, \mathrm{H} ; \mathrm{R}^{2}=\mathrm{O}$

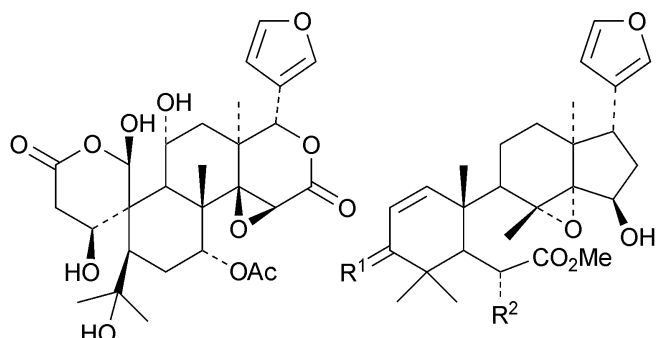

285
$286 \mathrm{R}^{1}=\alpha \mathrm{OH}, \mathrm{H} ; \mathrm{R}^{2}=\mathrm{OH}$ $287 \mathrm{R}^{1}=\mathrm{O} ; \mathrm{R}^{2}=\mathrm{OH}$ $288 R^{1}=O ; R^{2}=H$<smiles>CC(=O)CC1C(C)(C)C(O)C2OC2C1(C)C1CC(O)C23CC1(O)C2(C)C1OC1CC3c1ccoc1</smiles>

289

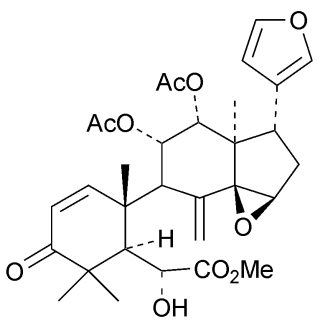

290

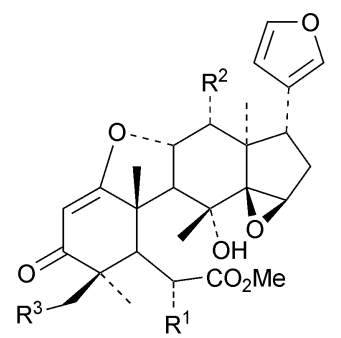

$291 \mathrm{R}^{1}=\mathrm{H} ; \mathrm{R}^{2}=\mathrm{OH} ; \mathrm{R}^{3}=\mathrm{H}$ $292 \mathrm{R}^{1}=\mathrm{OAC} ; \mathrm{R}^{2}=\mathrm{OH} ; \mathrm{R}^{3}=\mathrm{H}$ $293 \mathrm{R}^{1}=\mathrm{H} ; \mathrm{R}^{2}=\mathrm{R}^{3}=\mathrm{OAC}$ $294 \mathrm{R}^{1}=\mathrm{OAc} ; \mathrm{R}^{2}=\mathrm{OH} ; \mathrm{R}^{3}=\mathrm{H} ; 1$ 1, -dihydro $295 R^{1}=O A c ; R^{2}=O H ; R^{3}=O A c ; 1 \beta, 2$-dihydro $300 R^{1}=O A C ; R^{2}=R^{3}=H$

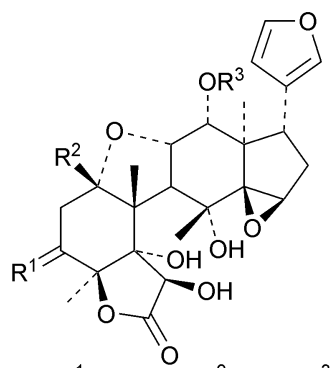

$296 \mathrm{R}^{1}=\beta \mathrm{OH}, \mathrm{H} ; \mathrm{R}^{2}=\mathrm{OH} ; \mathrm{R}^{3}=\mathrm{H}$ $297 \mathrm{R}^{1}=\alpha \mathrm{OH}, \mathrm{H} ; \mathrm{R}^{2}=\mathrm{OH} ; \mathrm{R}^{3}=\mathrm{H}$ $298 R^{1}=O ; R^{2}=H ; R^{3}=A C$ $299 \mathrm{R}^{1}=\alpha \mathrm{OH}, \mathrm{H} ; \mathrm{R}^{2}=\mathrm{H} ; \mathrm{R}^{3}=\mathrm{Ac}$

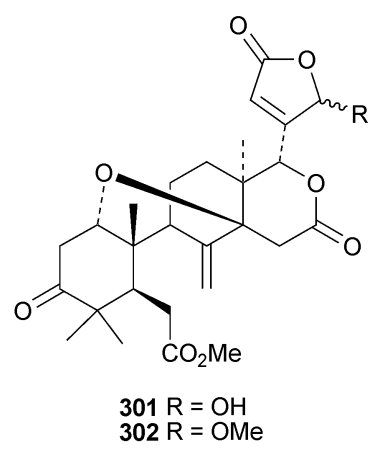

Four new ring C cleaved limonoids 307-310 have been isolated from the fruit of Melia toosendan, together with the tirucallane derivatives meliasenins S 311 and T 312. ${ }^{139}$ Meliasenin T 312 was also obtained from Melia azedarach seeds where it occurs with the tirucallane 313, the toosendanin esters 314 and 315 and the nimbolinin $\mathrm{C}$ derivative $316 .{ }^{\mathbf{1 4 0}}$ The ring-C cleaved hydroperoxide $\mathbf{3 1 7}$ has been isolated from Azadirachta indica. ${ }^{118}$

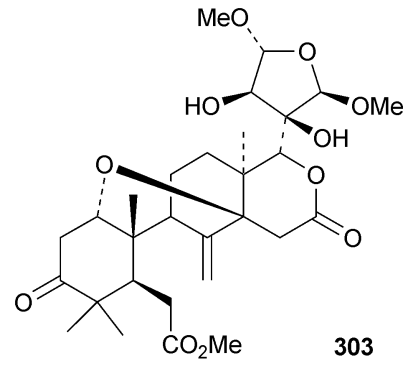

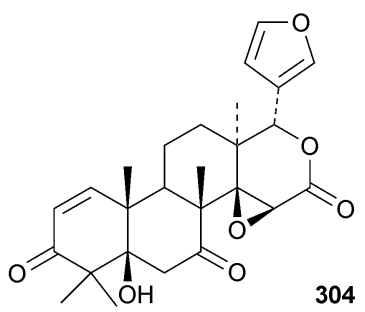

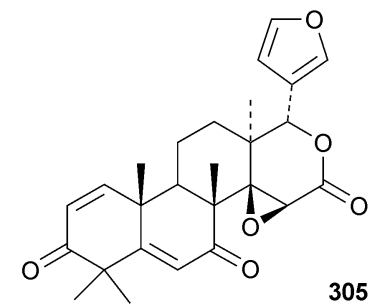<smiles>CC(=O)C[C@H]1C(C)(C)C=CC(=O)C1(C)C</smiles> 


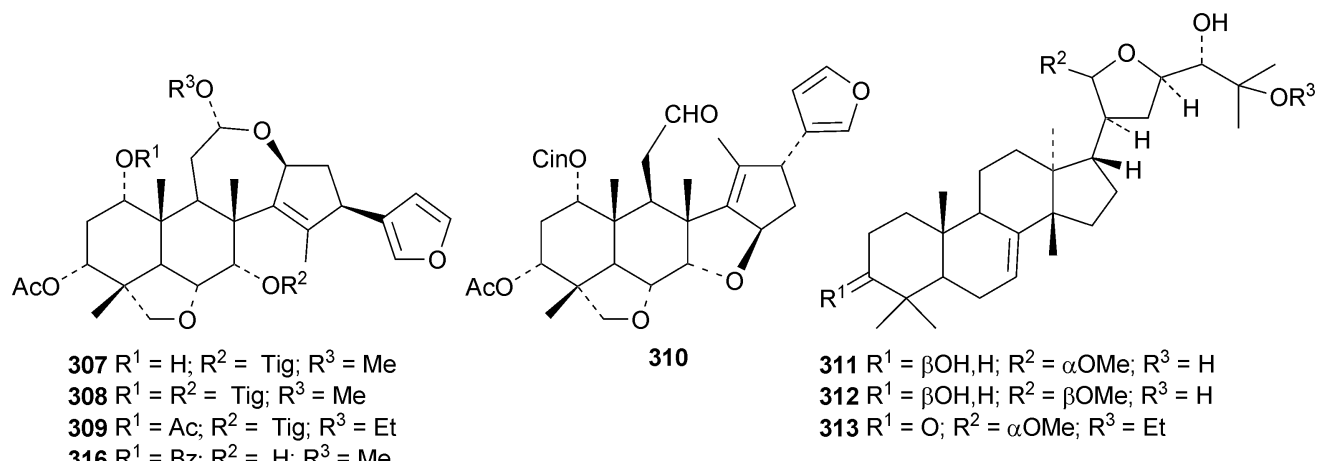

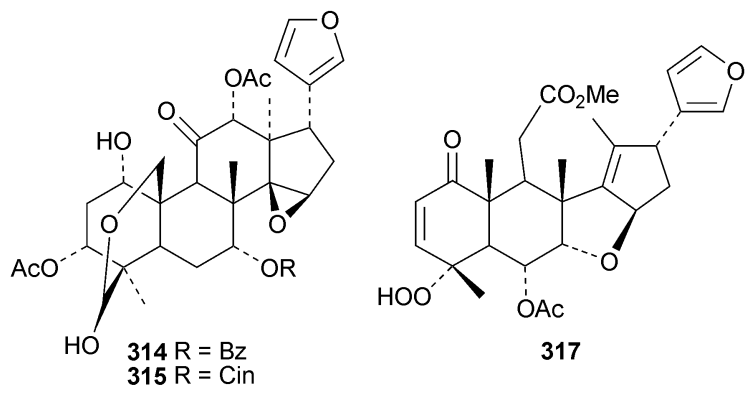

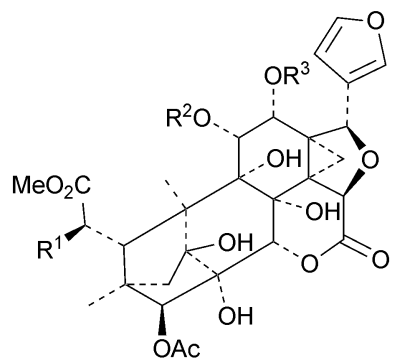

$318 \mathrm{R}^{1}=\mathrm{H} ; \mathrm{R}^{2}=\mathrm{OCCH}_{2} \mathrm{CH}_{3} ; \mathrm{R}^{3}=\mathrm{AC}$ $319 \mathrm{R}^{1}=\mathrm{H} ; \mathrm{R}^{2}=\mathrm{OCCH}\left(\mathrm{CH}_{3}\right)_{2} ; \mathrm{R}^{3}=\mathrm{AC}$ $320 \mathrm{R}^{1}=\mathrm{OAC} ; \mathrm{R}^{2}=\mathrm{Ac} ; \mathrm{R}^{3}=\mathrm{OCCH}\left(\mathrm{CH}_{3}\right)_{2}$ $321 \mathrm{R}^{1}=\mathrm{OAC} ; \mathrm{R}^{2}=\mathrm{H} ; \mathrm{R}^{3}=\mathrm{OCCH}\left(\mathrm{CH}_{3}\right)_{2}$ $322 \mathrm{R}^{1}=\mathrm{OAC} ; \mathrm{R}^{2}=\mathrm{OCCH}\left(\mathrm{CH}_{3}\right)_{2} ; \mathrm{R}^{3}=\mathrm{AC}$ $323 \mathrm{R}^{1}=\mathrm{OAC} ; \mathrm{R}^{2}=\mathrm{R}^{3}=A C$

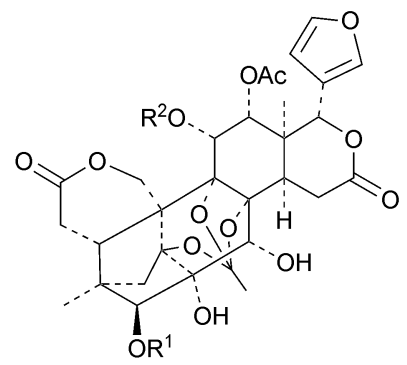

$330 \mathrm{R}^{1}=\mathrm{R}^{2}=\mathrm{H}$

$331 \mathrm{R}^{1}=\mathrm{Ac} ; \mathrm{R}^{2}=\mathrm{H}$ $332 \mathrm{R}^{1}=\mathrm{H} ; \mathrm{R}^{2}=\mathrm{AC}$

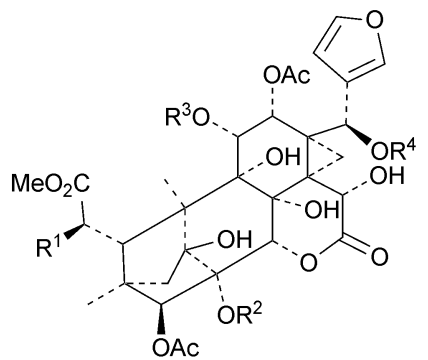

$324 \mathrm{R}^{1}=\mathrm{OAC} ; \mathrm{R}^{2}=\mathrm{H} ; \mathrm{R}^{3}=\mathrm{Ac} ; \mathrm{R}^{4}=\mathrm{OCCH}\left(\mathrm{CH}_{3}\right)_{2}$ $325 \mathrm{R}^{1}=\mathrm{OACR} \mathrm{R}^{2}=\mathrm{H} ; \mathrm{R}^{3}=\mathrm{OCCH}\left(\mathrm{CH}_{3}\right)_{2} ; \mathrm{R}^{4}=\mathrm{AC}$ $326 \mathrm{R}^{1}=\mathrm{OAC} ; \mathrm{R}^{2}=\mathrm{OCCH}\left(\mathrm{CH}_{3}\right)_{2} ; \mathrm{R}^{3}=\mathrm{R}^{4}=\mathrm{AC}$ $327 R^{1}=H ; R^{2}=H ; R^{3}=A C ; R^{4}=\operatorname{OCCH}\left(C_{3}\right)_{2}$

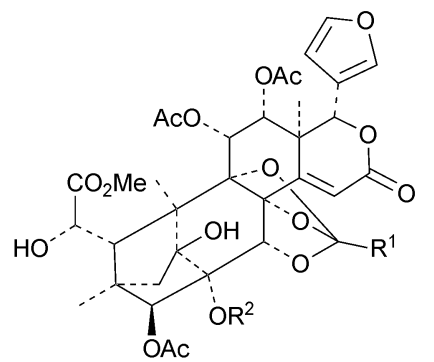

$328 \mathrm{R}^{1}=\mathrm{CH}\left(\mathrm{CH}_{2}\right)_{3} ; \mathrm{R}^{2}=\mathrm{H}$ $329 \mathrm{R}^{1}=\mathrm{Me} ; \mathrm{R}^{2}=\mathrm{OCCH}\left(\mathrm{CH}_{3}\right)_{2}$

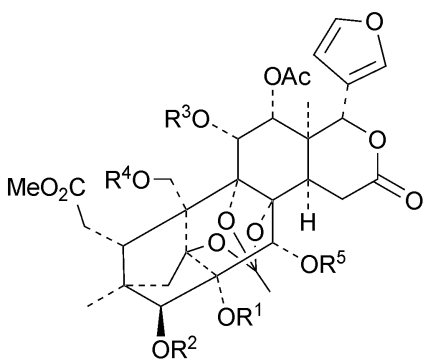

$333 R^{1}=R^{2}=R^{4}=R^{5}=A c ; R^{3}=H$ $334 \mathrm{R}^{1}=\mathrm{H} ; \mathrm{R}^{2}=\mathrm{R}^{3}=\mathrm{R}^{4}=\mathrm{R}^{5}=A C$ $335 R^{1}=R^{2}=R^{5}=H ; R^{3}=R^{4}=A C$ $336 R^{1}=R^{3}=R^{5}=H ; R^{2}=A c ; R^{4}=\operatorname{OCCH}\left(C_{3}\right)_{2}$

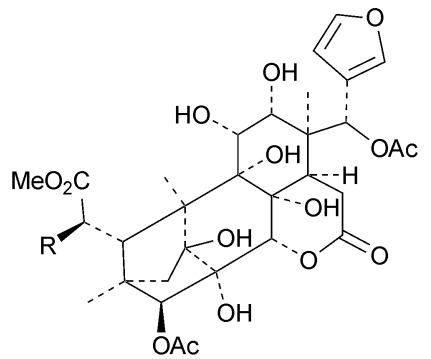

$337 \mathrm{R}=\mathrm{H}$ $338 \mathrm{R}=\mathrm{OAC}$
The flow of new mexicanolide and phragmalin derivatives continues unabated. Chukrasia tabularis var. velutina is a particularly rich source. The new derivatives reported from this source include velutabularins A-J 318-327, ${ }^{141}$ tabulalides F-N 328-336, ${ }^{142}$ tabulalins A-E 337-341, ${ }^{143}$ chukvelutilide $\mathrm{H} 342$ and tabularisin R 343, ${ }^{144}$ tabulvelutins A 344 and B $345^{145}$ and 
tabulalin F 346. ${ }^{\mathbf{1 4 6}}$ Many of these compounds are trivial variants of known systems. Velutabularins A-J 318-327 are cyclopropyl derivatives with a modified ring $\mathrm{D}$ and tabulvelutin $\mathrm{A}$ 344 is a 19-nor derivative. A similar range of phragmalin derivatives, swietenitins $\mathrm{N}-\mathrm{X}$ 347-357, has been isolated from the twigs of Swietenia macrophylla. ${ }^{\mathbf{1 4 7}}$ The structure of swietenitin N 347 was confirmed by X-ray analysis. The stereochemistry of the known compound 14,15-dihydroepoxyfebrinin B 358 was also established during this study. The leaves of Trichilia connaroides produced trichagmalins A-F 359-364 and several acetyl derivatives 365-369, together with trichanolide $370 .{ }^{148}$ The gedunin andirolide A 371, the mexicanolides andirolides B-D 372-374 and the phragmalins andirolides E-G 375-377 have been reported from the flowers

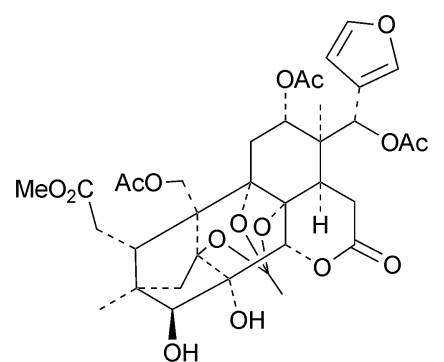

339

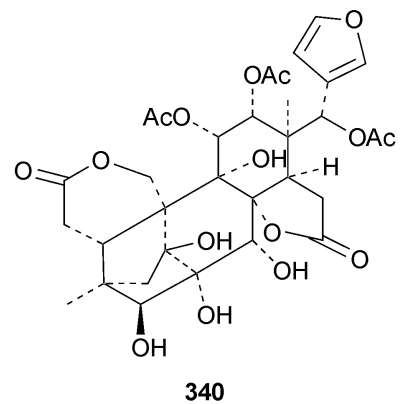

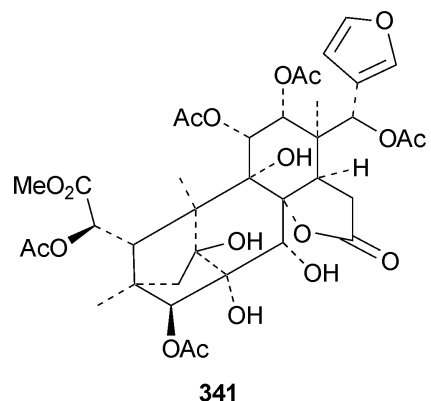

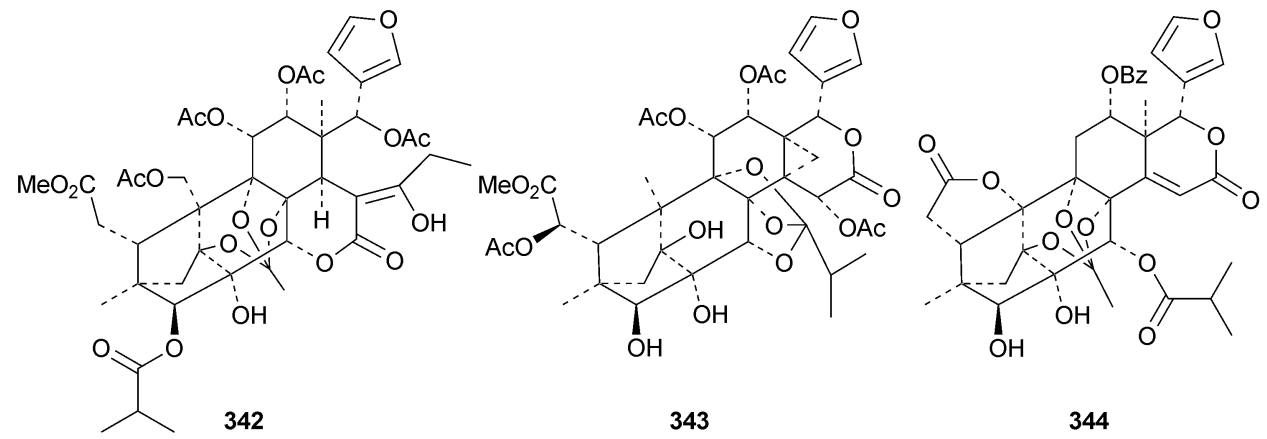

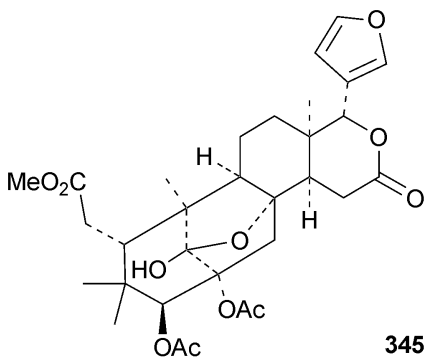

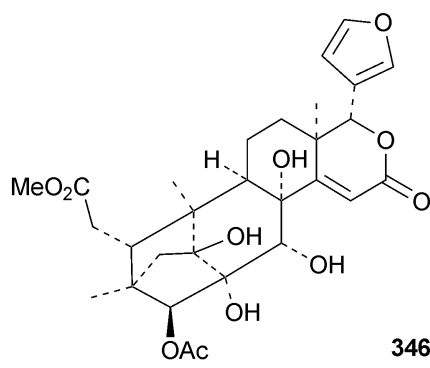
345

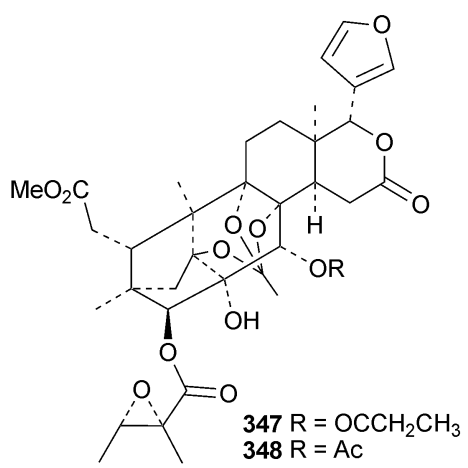

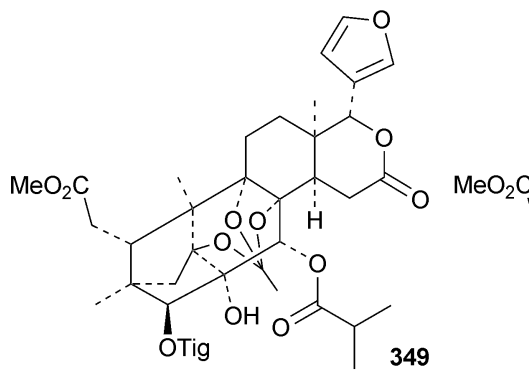

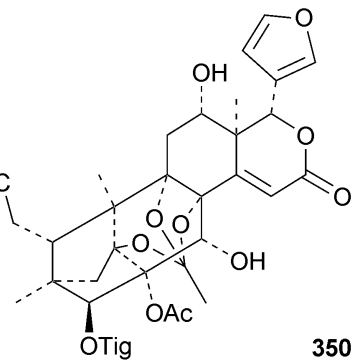

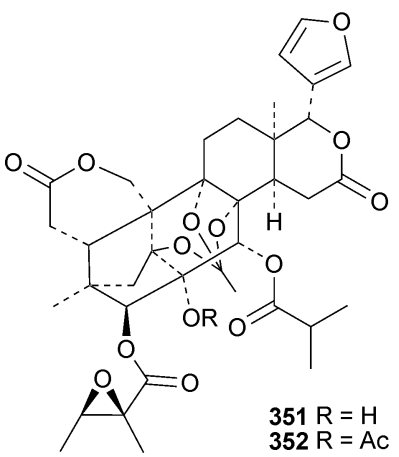




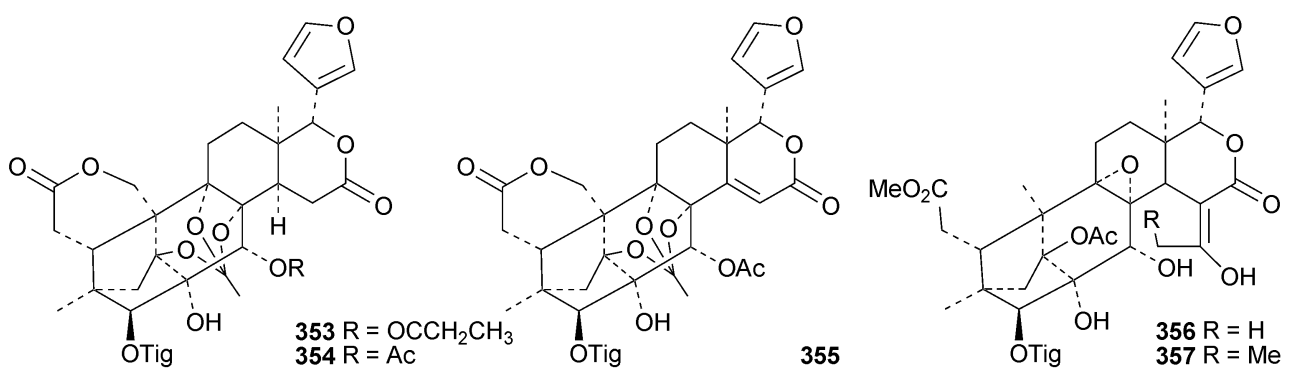

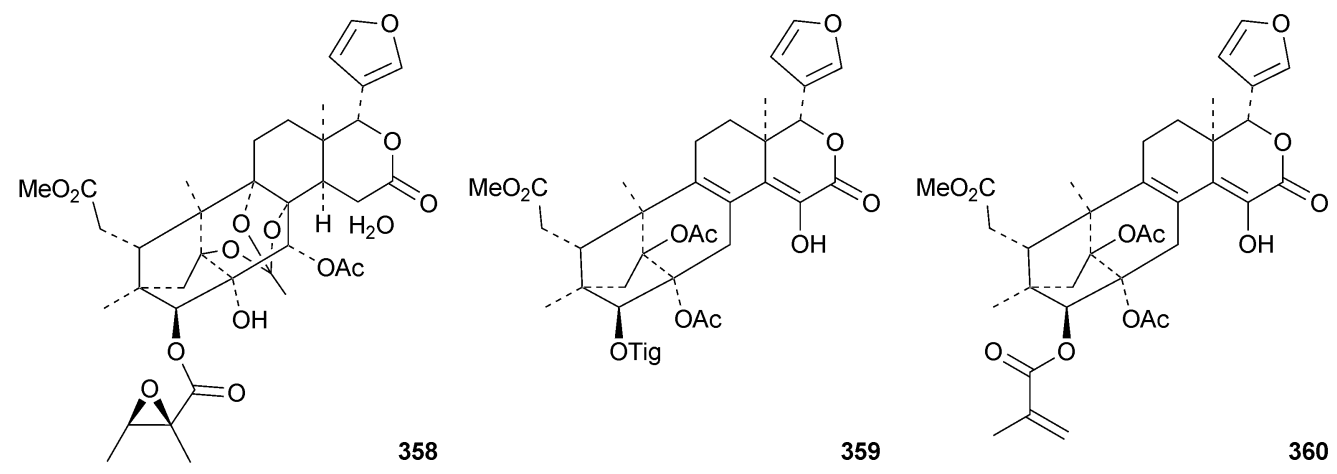

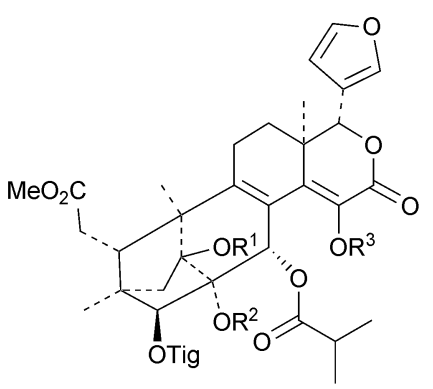

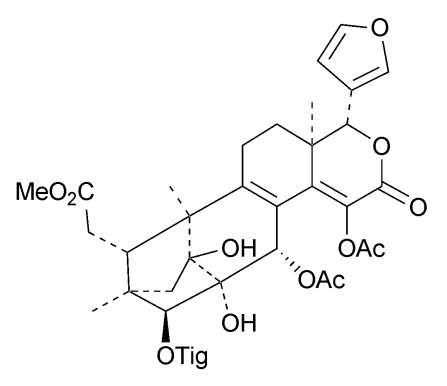

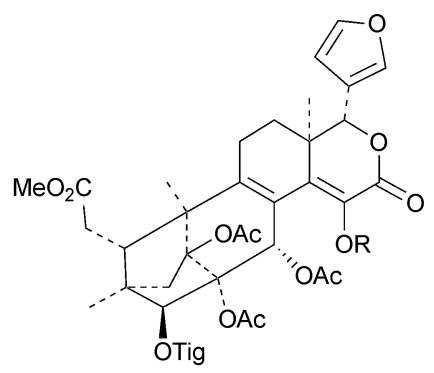

$361 R^{1}=R^{2}=R^{3}=H$ $365 \mathrm{R}^{1}=\mathrm{R}^{2}=\mathrm{H} ; \mathrm{R}^{3}=A C$ 362 $363 R=H$
$367 R=A C$ $366 R^{1}=R^{2}=A C ; R^{3}=H$

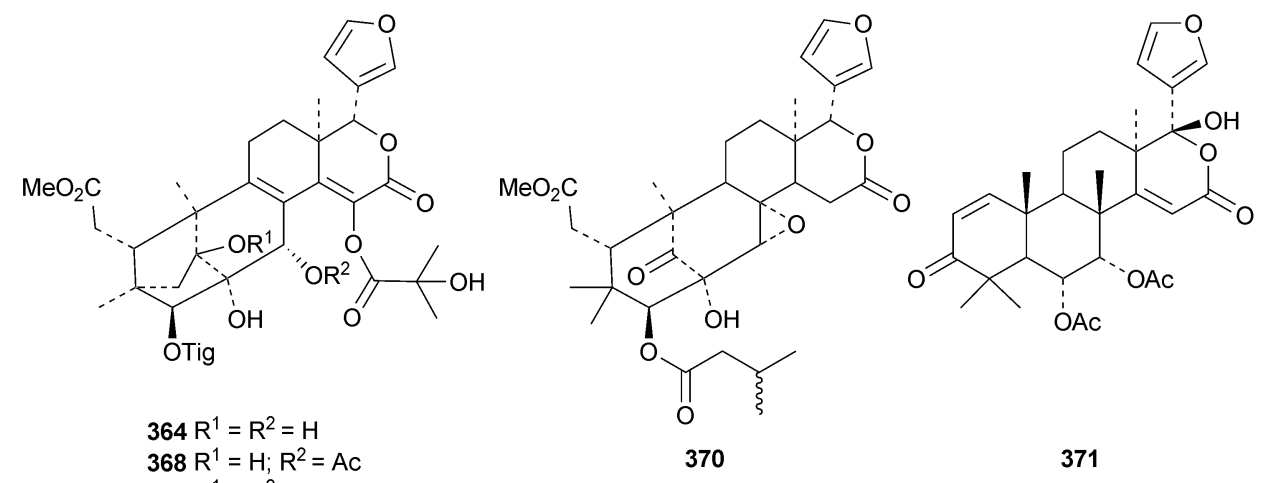

of Carapa guianensis. ${ }^{149}$ The structure of andirolide E $\mathbf{3 7 5}$ was confirmed by X-ray analysis. Other phragmalin derivatives include thaimoluccensins B $\mathbf{3 7 8}$ and C $\mathbf{3 7 9}$ from the seeds of Thai Xylocarpus moluccensis ${ }^{\mathbf{1 3 8}}$ and godvarin $\mathrm{K} \mathbf{3 8 0}$ from the Godvari mangrove Xylocarpus moluccensis. ${ }^{150}$
New quassinoids are few in number. They include 2 '-isopicrasin A 381 from the stems of Picrasma quassinoides, ${ }^{151}$ bruceines K 382 and L 383 from the ripe fruit of Brucea javanica, ${ }^{\mathbf{1 5 2}}$ yadanziolides T-V 384-386 from the stems of Brucea mollis ${ }^{153}$ and nothospondin $\mathbf{3 8 7}$ from Nothospondias staudtii. ${ }^{\mathbf{1 5 4}}$ 


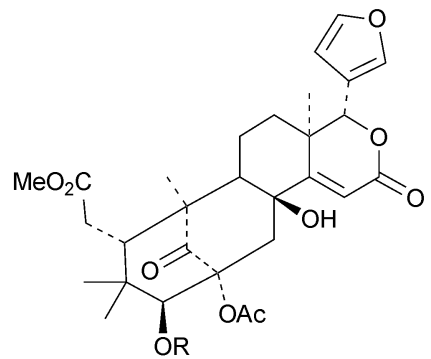

$372 \mathrm{R}=\mathrm{Ac}$ $373 \mathrm{R}=\mathrm{OCCH}\left(\mathrm{CH}_{3}\right)_{2}$ $374 \mathrm{R}=\mathrm{Tig}$

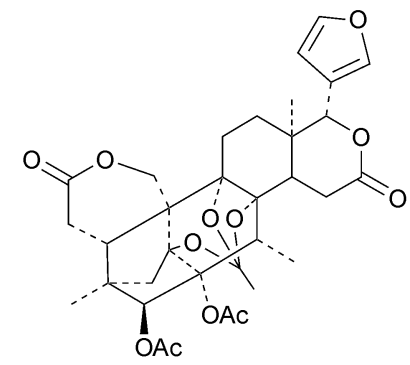

376 14,15-didehydro

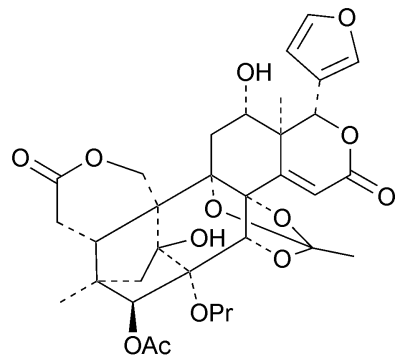

377

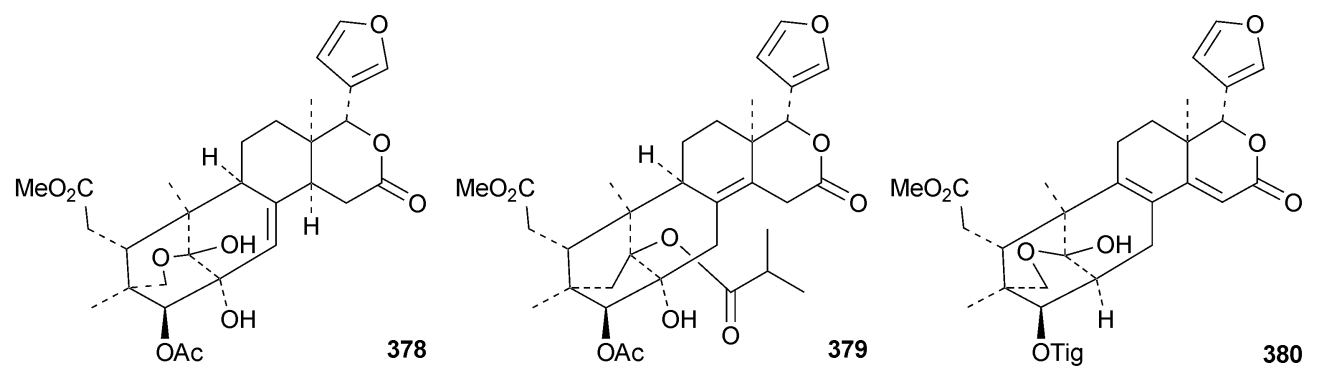

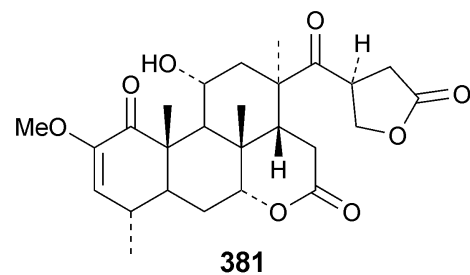

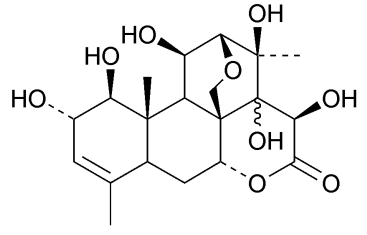

382

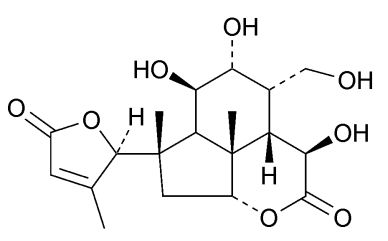

386

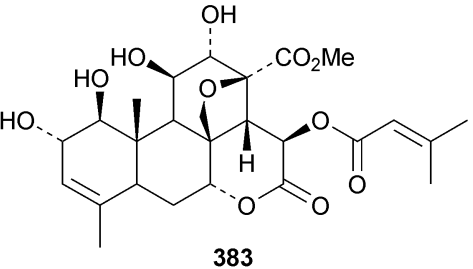

383

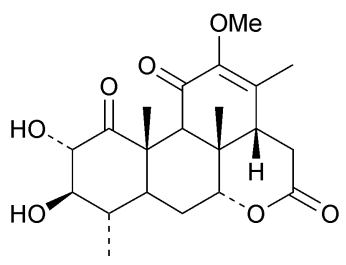

387 $385 \mathrm{R}=\mathrm{GlC}$
$384 \mathrm{R}-\mathrm{H}$

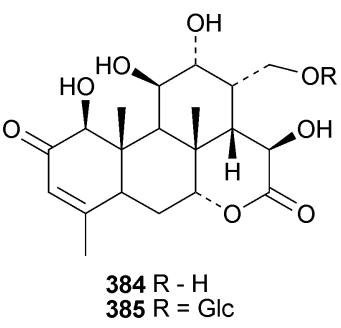




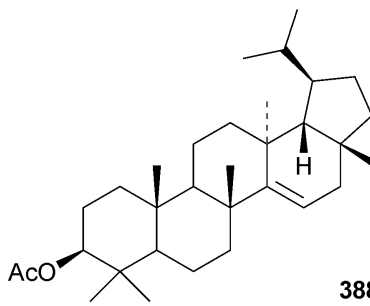<smiles>CC(=O)O[C@H]1CC[C@]2(C)C3CC[C@H]4C(C(C)C)=CC[C@]4(C)CC[C@]3(C)[C@]2(C)CCC1C</smiles>

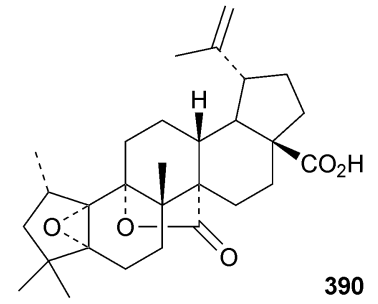<smiles>C=C(C)[C@H]1CC[C@]2(C)C(CC[C@@]3(C)[C@H]4C(C(C)(C)O)CC[C@@]4(C)CC[C@H]23)[C@]1(C)CCC(=O)O</smiles>

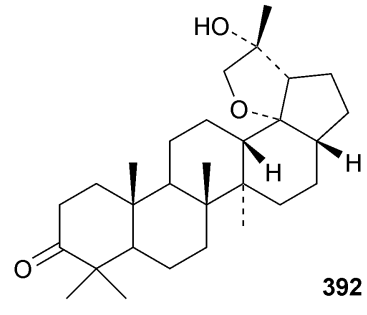

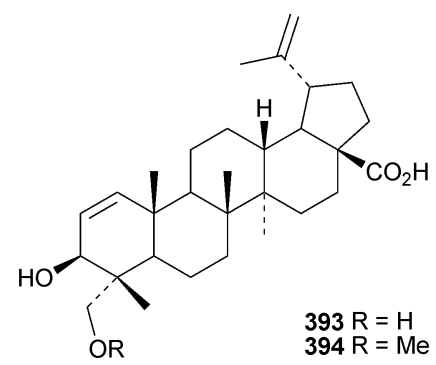

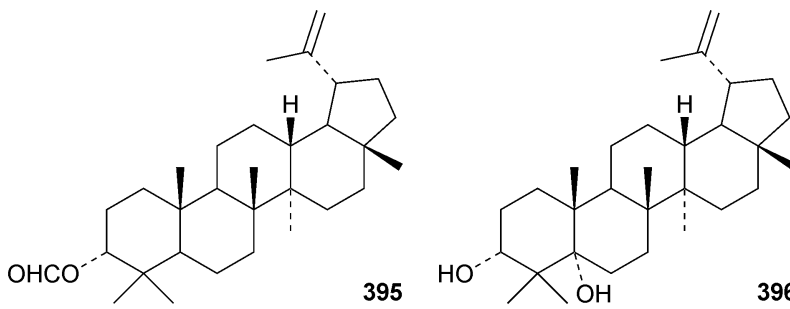

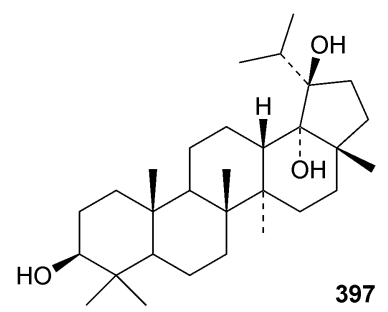<smiles>CC(C)C1CC[C@]2(CO)CC[C@]3(C)C(=CCC4[C@@]5(C)CC[C@@H](O)C(C)(C)C5CC[C@]43C)C12</smiles>

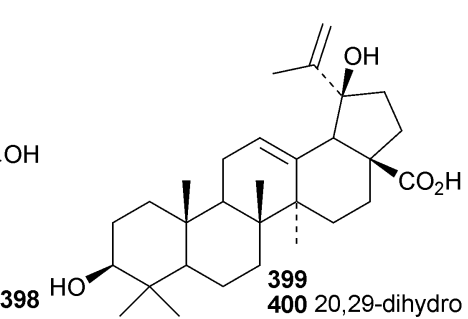<smiles>C=C(C)C1CCC2(C(=O)O)CCC3(C(=O)O)C(CCC4[C@@]5(C)CC[C@H](O)[C@](C)(C(=O)O)C5CC[C@]43C)[C@H]12</smiles>

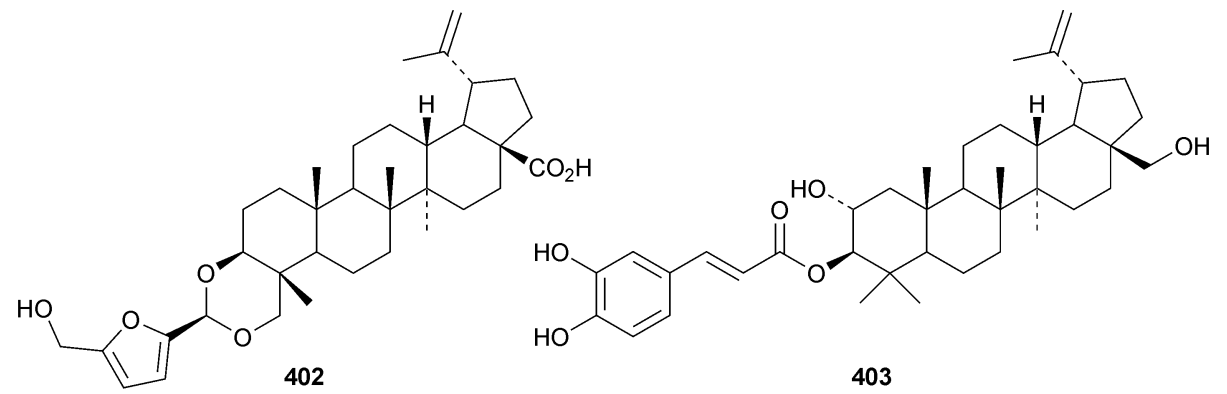

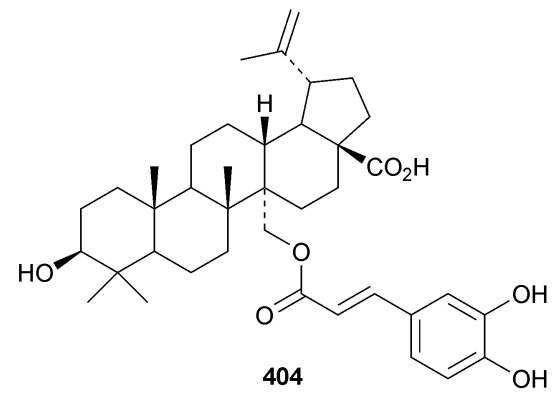

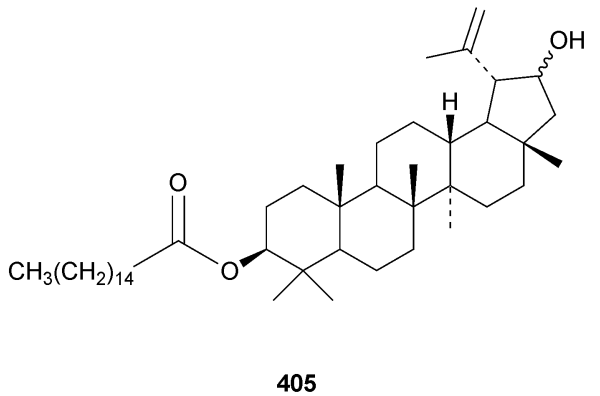




\section{The oleanane group}

Several ring-A seco-oleanane triterpenoids have been isolated, including the 2,3-seco-oleanenetrioic acid 406 from Dillenia philippinensis, ${ }^{172}$ dysoxyhainic acid F 407, G 408, I 409 and J 410 from Dysoxylum hainanense, ${ }^{159}$ the 12-ketone 411 and 13(18)-ene 412 from Betula pendula, ${ }^{173}$ the 3-methyl ester 413 from Kalopanax pictus ${ }^{\mathbf{1 7 4}}$ and ivorengenin B 414 from Terminalia ivorensis. ${ }^{175}$ The unusual 9,25-cycloolean-12-en-3 $\beta$-yl $\beta$-D-glucofuranoside 415 has been reported to be a constituent of Celestris australis ${ }^{\mathbf{1 7 6}}$ and the same group has identified 9,25-cyclo-3 $\beta$-( $\beta$-D-glucopyranosyloxy)-16 $\alpha$-hydroxyolean-12-en-28-oic acid 416 in Symplocos paniculata. ${ }^{177}$ The 24,30-dinoroleanane 417, 30-noroleanane 418 and 24-noroleanane 419 derivatives are present in the roots of Paeonia rockii ssp. rockii. ${ }^{178}$ A review covering the structures and pharmacological activity of noroleanane triterpenoids has been published. ${ }^{179}$ The antitumour activities of oleanane triterpenoids have been surveyed. ${ }^{180}$

Fatsicarpains A-G 420-426 are oleanane derivatives from leaves and twigs of Fatsia polycarpa. ${ }^{181}$ The structures of fatsicarpain A 420 and the co-occurring known oleananes 427 and 428 were confirmed by X-ray analyses. 15 $\alpha$-Hydroxysoyasapogenol B 429, 7 $\beta, 15 \alpha$-hydroxysoyasapogenol B 430 and $7 \beta$,29-dihydroxysoyasapogenol $\mathbf{4 3 1}$ are metabolites of the

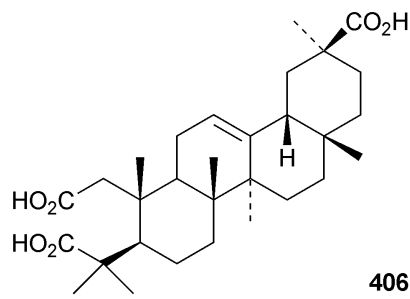<smiles>CC(C)(C(=O)O)C1CC[C@]2(C)C(CC=C3[C@@]4(C)C(C)(C(=O)O)CC[C@@]4(C)CC[C@]32C)[C@@]1(C)C(=O)O</smiles>

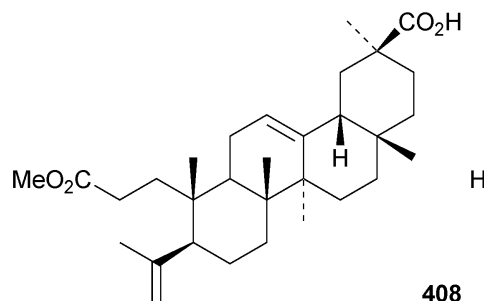

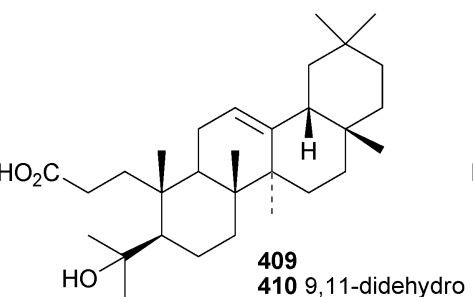<smiles>C=C(C)[C@H]1CC[C@@]2(C)[C@H]3CC[C@@]4(C)CCC(C)(C)C[C@@H]4[C@H]3C(=O)C[C@H]2[C@]1(C)CCC(=O)O</smiles><smiles>C=C(C)[C@H]1CC[C@]2(C)C3CCC4=C5CC(C)(C)CC[C@]5(COC(C)=O)CCC4[C@@]3(C)CCC2[C@]1(C)CCC(=O)O</smiles>

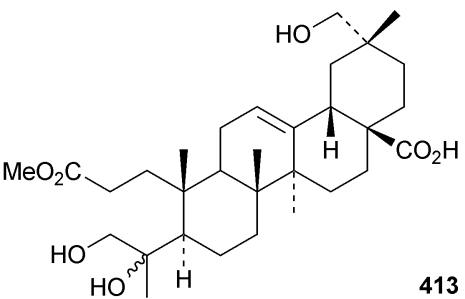

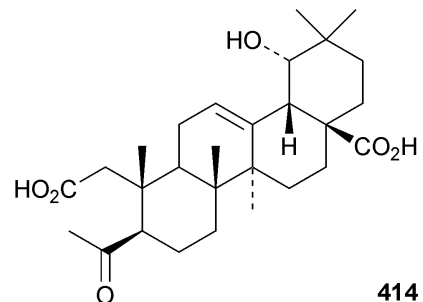

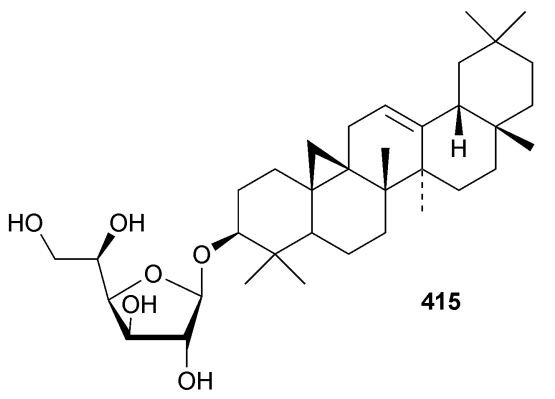

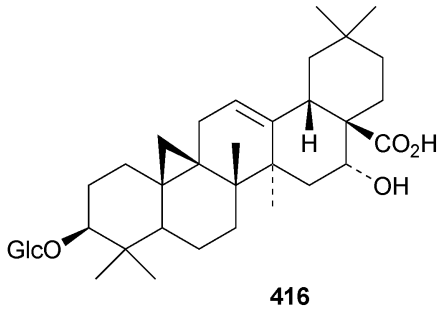

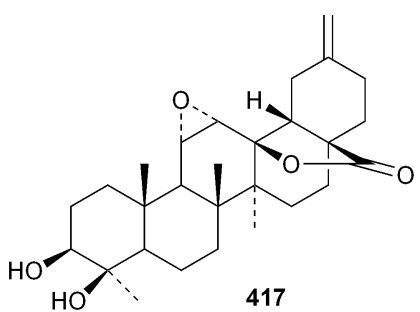<smiles></smiles><smiles>CC1(C)CCC2(C(=O)O)CCC3(C)C(=CCC4C5(C)CC[C@@H](O)[C@](O)(CO)C5CC[C@]43C)C2(C)C1</smiles> 


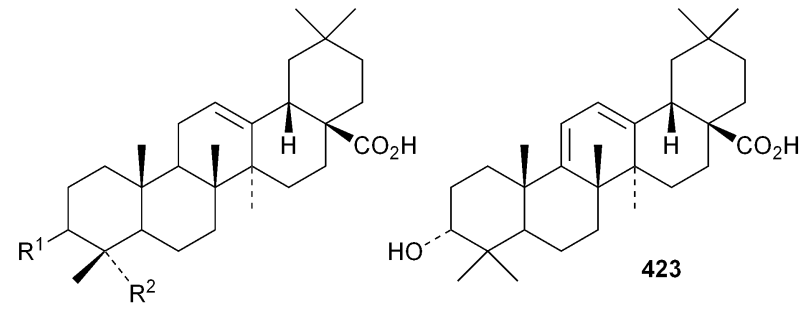

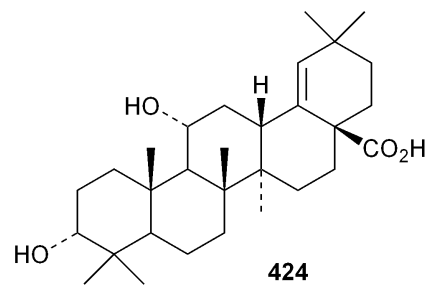

$420 \mathrm{R}^{1}=\alpha \mathrm{OH} ; \mathrm{R}^{2}=\mathrm{CH}_{2} \mathrm{OH}$ $421 \mathrm{R}^{1}=\mathrm{BOSO}_{3} \mathrm{H} ; \mathrm{R}^{2}=\mathrm{CH}_{3}$ $422 \mathrm{R}^{1}=\alpha \mathrm{OH} ; \mathrm{R}^{2}=\mathrm{CHO}$ $427 \mathrm{R}^{1}=\alpha \mathrm{OH} ; \mathrm{R}^{2}=\mathrm{CH}_{3}$

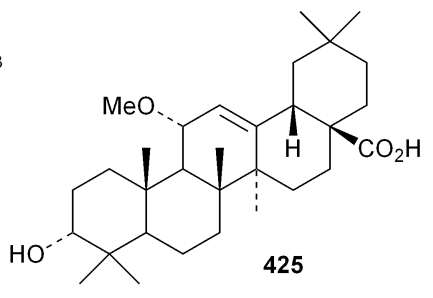

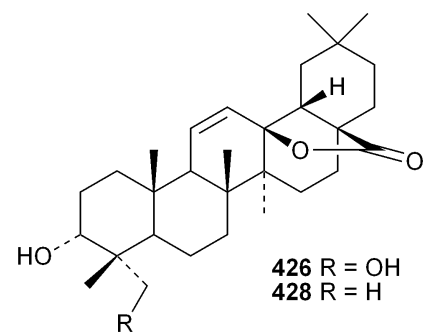

endophytic fungus Pestalotiopsis clavispora, isolated from Bruguiera sexangula. ${ }^{182}$ The structure of $15 \alpha$-hydroxysoyasapogenol B 429 was confirmed by X-ray analysis. The structure of olean13(18)-ene-3,12,19-trione 432, from Sedum linare, was also established by X-ray analysis. ${ }^{183}$

Other new simple oleanane derivatives include ambradiolic acid A 433 from Liquidambar formosana, ${ }^{160}$ 16 $16,23,29$-trihydroxy- 3-oxoolean-12-en-28-oic acid $\mathbf{4 3 4}$ from Kalopanax pictus, ${ }^{174}$ ivorengenin A $\mathbf{4 3 5}$ from Terminalia ivorensis, ${ }^{175}$ salacetal 436 from Salacia longipes var. camerunensis, ${ }^{184}$ olean-12-ene-3 $\alpha, 23-$ diol $\mathbf{4 3 7}$ from Salvia miltiorrhiza, ${ }^{185}$ camelliagenone $\mathbf{4 3 8}$ from Barringtonia asiatica, ${ }^{186}$ the 1,3-diols $\mathbf{4 3 9}$ and $\mathbf{4 4 0}$ from Viburnum chingii, ${ }^{187} 2 \alpha, 3 \alpha, 19 \alpha, 23$-tetrahydroxyolean-12-en-28-oic acid $\mathbf{4 4 1}$ from Rosa laevigata, ${ }^{188} 3 \beta$-hydroxyolean-18-en-1-one 442 from

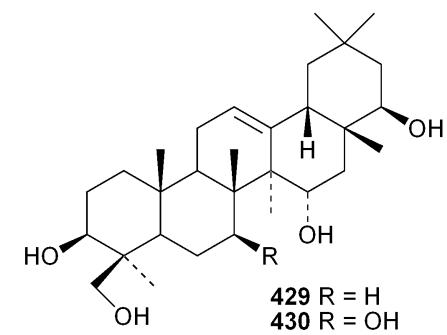

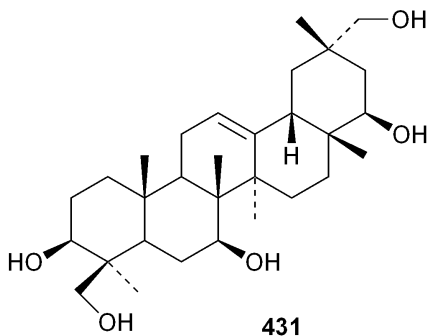<smiles>CC1(C)CCC(=O)C(C23CCC4C(C)(C)CCC(C)(C)C4(C)CCC2C2=C3C(C)(C)CCC2(C)C)CC1=O</smiles>

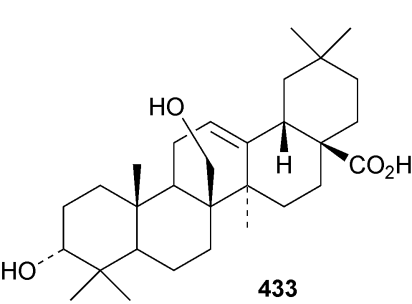<smiles>CC1(CO)CCC2(C)C(C(O)CC3=CCC4C5(C)CCC(=O)C(C)(CO)C5CCC4(C)C32)C1CO</smiles>

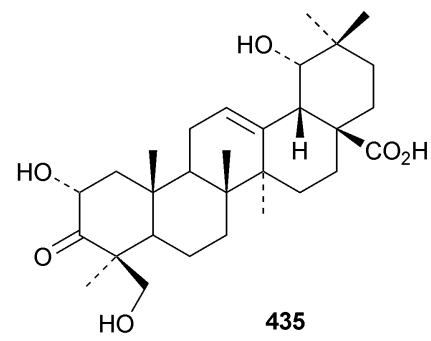

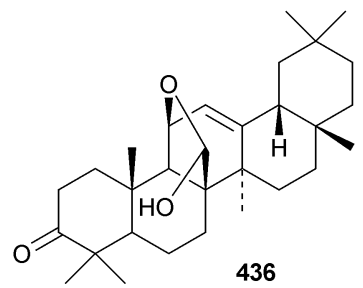<smiles>CC1(C)CCC2(C)C3CCC4C(C)(CCC5C4(C)CC[C@H](O)C5(C)CO)C3CCC2(C)C1</smiles><smiles>CC1(C)C(=O)CC[C@@]2(C)C1CC[C@]1(C)C2CC=C2C3(C)C(C)(C)C[C@H](O)[C@]3(CO)C(O)C[C@]21C</smiles> 


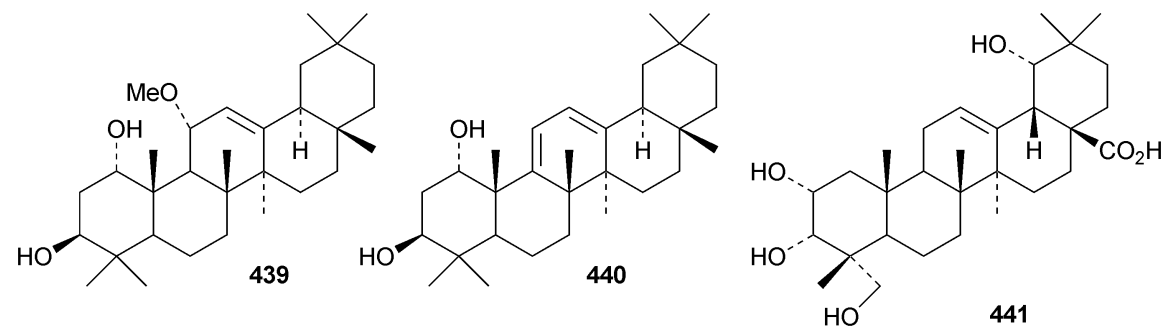<smiles>CC1(C)[C@@H]2CC[C@]3(C)C4=CC(C)(C)C(C)(C)CC[C@H]4CCC3[C@@]2(C)C(=O)C[C@H]1O</smiles>

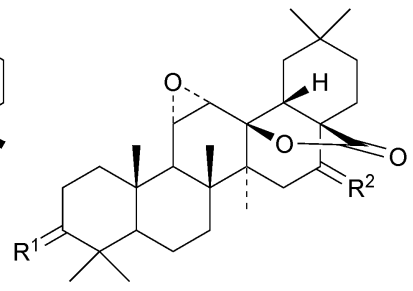

$443 \mathrm{R}^{1}=\beta \mathrm{OH}, \mathrm{H} ; \mathrm{R}^{2}=\alpha \mathrm{OH}, \mathrm{H}$ $444 \mathrm{R}^{1}=\mathrm{O} ; \mathrm{R}^{2}=\alpha \mathrm{OH}, \mathrm{H}$ $445 \mathrm{R}^{1}=\beta \mathrm{OH}, \mathrm{H} ; \mathrm{R}^{2}=\mathrm{O}$

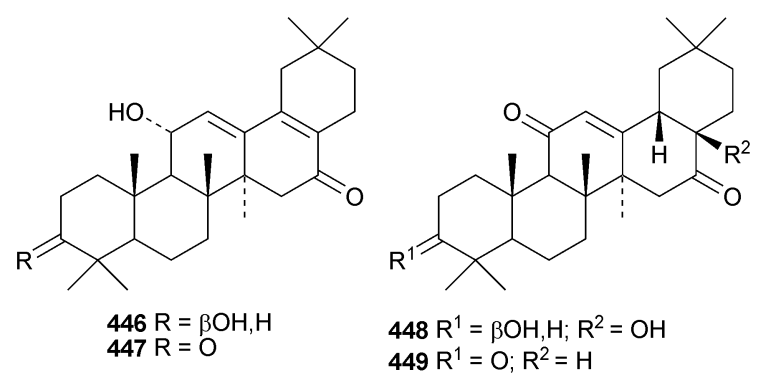

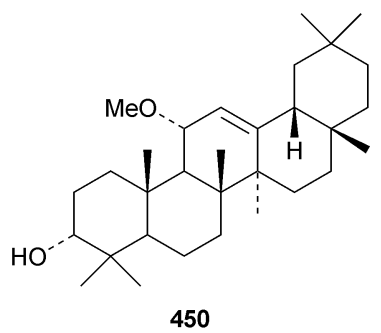

Juglans chinensis, ${ }^{\mathbf{1 8 9}}$ 443-449 from Nannoglottis carpesioides ${ }^{\mathbf{1 9 0}}$ and olibanumol E 450 from olibanum, the gum resin of Boswellia carterii. ${ }^{\mathbf{1 6 2}}$

Pulsatilla triterpenic acids B 451 and C 452, from Pulsatilla chinensis, are hederagenin acetal derivatives. ${ }^{167}$ The first glycyrrhetic acid amino acid conjugate, dendrophen 453 , has been

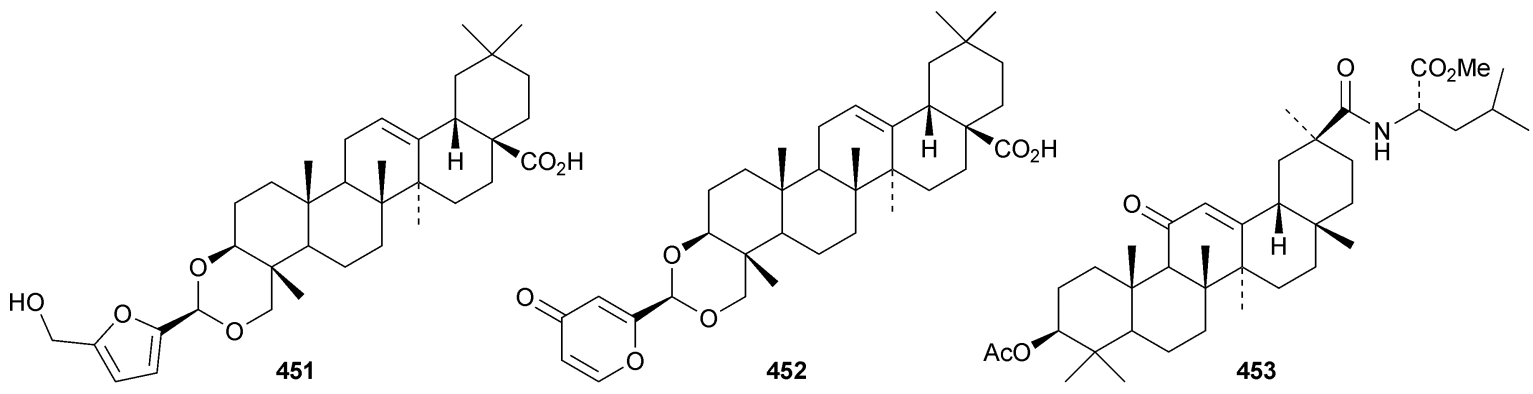

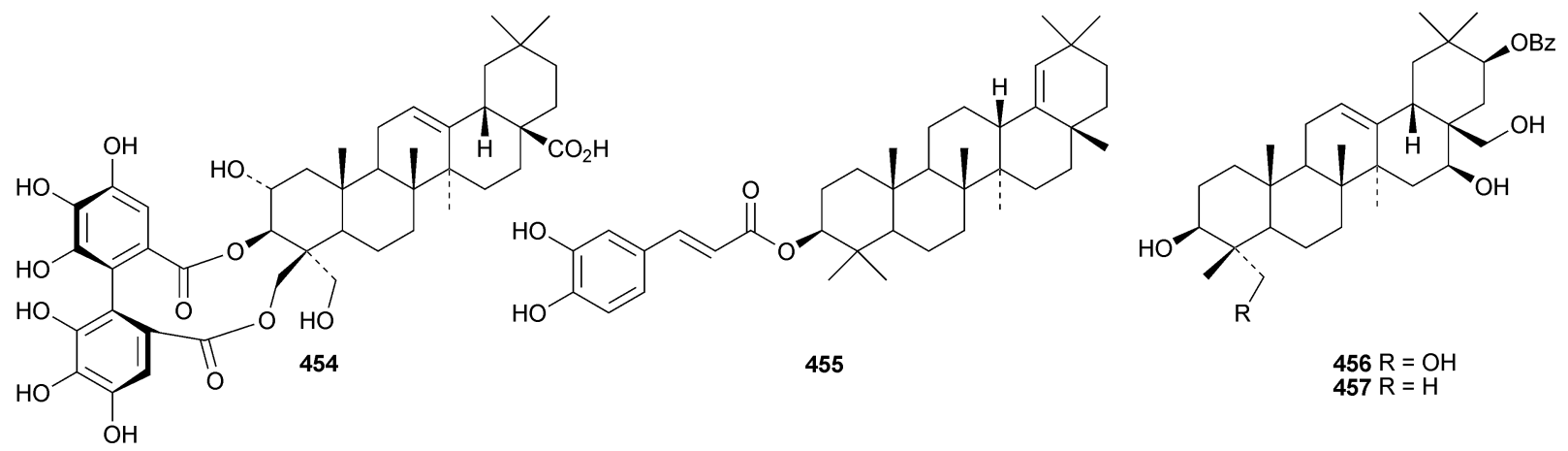




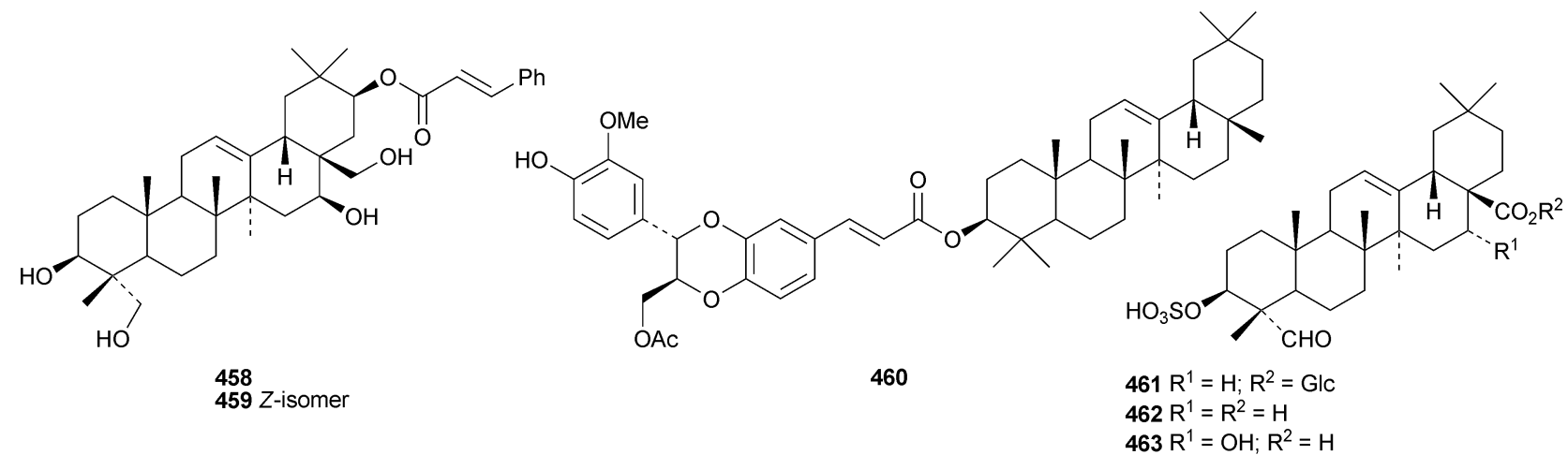

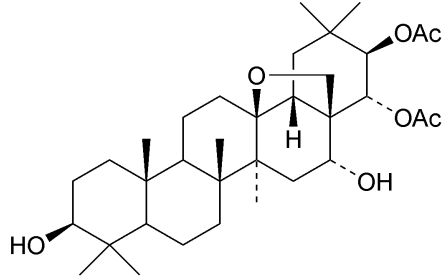

464

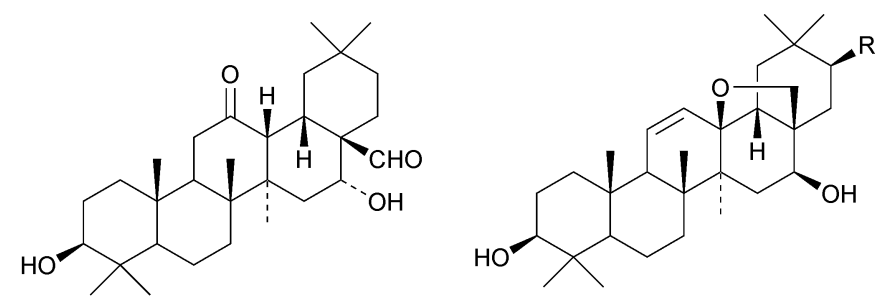

465<smiles>CCC(C)C(=O)NCCCC(=O)OCC(=O)O</smiles>

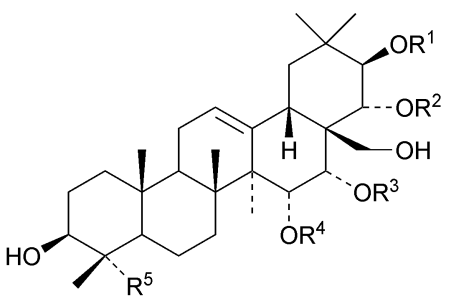

$468 \mathrm{R}^{1}=\mathrm{R}^{3}=\mathrm{R}^{4}=\mathrm{Ac} ; \mathrm{R}^{2}=$ 2-methylbutanoyl; $\mathrm{R}^{5}=\mathrm{CH}_{2} \mathrm{OH}$ $469 R^{1}=R^{3}=R^{4}=A c ; R^{2}=$ angeloyl; $R^{5}=\mathrm{CH}_{2} \mathrm{OH}$ $470 \mathrm{R}^{1}=$ angeloyl; $\mathrm{R}^{2}=2$-methylbutanoyl; $\mathrm{R}^{3}=\mathrm{Ac} ; \mathrm{R}^{4}=\mathrm{H} ; \mathrm{R}^{5}=\mathrm{CH}_{2} \mathrm{OH}$ $471 \mathrm{R}^{1}=$ angeloyl; $\mathrm{R}^{2}=2$-methylbutanoyl; $\mathrm{R}^{3}=\mathrm{R}^{4}=\mathrm{H} ; \mathrm{R}^{5}=\mathrm{CHO}$ $472 \mathrm{R}^{1}=$ angeloyl; $\mathrm{R}^{2}=2$-methylbutanoyl; $\mathrm{R}^{3}=\mathrm{Ac} ; \mathrm{R}^{4}=\mathrm{H} ; \mathrm{R}^{5}=\mathrm{CHO}$ $473 \mathrm{R}^{1}=$ angeloyl; $\mathrm{R}^{2}=2$-methylbutanoyl; $\mathrm{R}^{3}=\mathrm{Ac} ; \mathrm{R}^{4}=\mathrm{H} ; \mathrm{R}^{5}=\mathrm{CO}_{2} \mathrm{Me}$

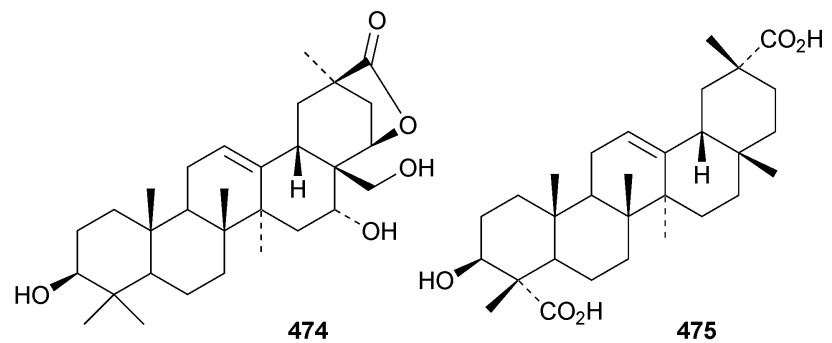

474

475 isolated from Dendronephthya hemprichi. ${ }^{191}$ The aglycone 454 of the known castanopsinin $\mathrm{E}_{\mathrm{a}}$ has been found in leaves of Castanopsis fissa. ${ }^{192}$ Other new oleanane ester derivatives include the caffeoyl ester of germanicol 455 from Barringtonia asiat$i c a,{ }^{\mathbf{1 8 6}}$ esters 456-459 from Glochidion assamicum, ${ }^{193}$ uragogin 460 from Crossopetalum uragoga, ${ }^{194}$ and the sulfate esters 461463 from Gypsolphila pacifica. ${ }^{195}$

Clethroidosides A-G are oleanane saponins from Lysimachia clethroides. ${ }^{196}$ Clethroidosides $\mathrm{F}$ and $\mathrm{G}$ have the new genins 464 and 465, respectively; the others have known genins. Heterogenoside F, from Lysimachia heterogenea, is identical to clethroidoside $\mathrm{F}$ and it is found with heterogenoside $\mathrm{E}$ that has a known genin. ${ }^{197}$ The genins of glaucasides A and B, from Atriplex glauca var. ifiniensis, are the new compounds 466 and 467 whereas glaucaside $\mathrm{C}$ has the known genin saikogenin G. Camellia sinensis is a rich source of saponins including rogchaponins R1-R10. ${ }^{198}$ Rogchaponins R1, R2 and R4-R7 have the new genins 468-473, respectively. Myrseguinoside D, from Myrsine seguinii, has the new genin $\mathbf{4 7 4} .{ }^{199}$ It is accompanied by myrseguinoside $\mathrm{E}$ which is the same as the known ardisicrenoside J. Dianthosaponins A-F are found in Dianthus japonicus. ${ }^{200}$ Dianthosaponins $\mathrm{E}$ and $\mathrm{F}$ have the new genins 475 and 476, respectively. Bridgesides A1, C1, C2, D1, D2, E1 and E2, from Echinopsis macrogona, include the new genins 477 and 


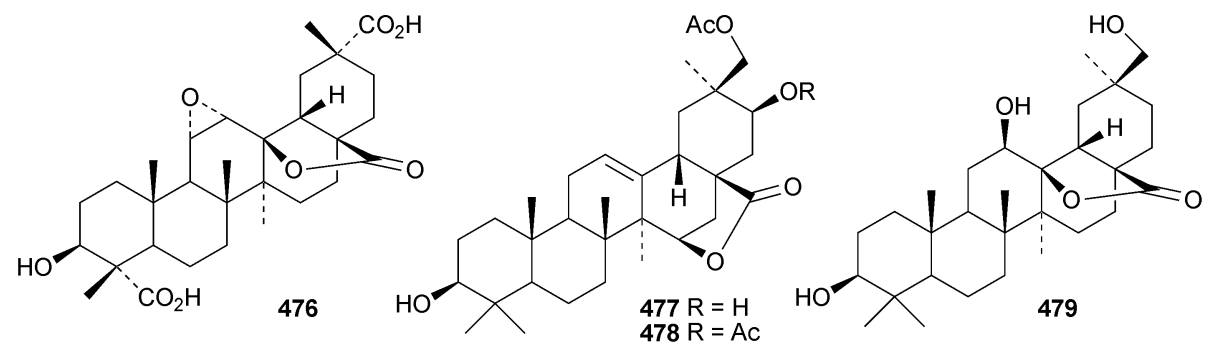

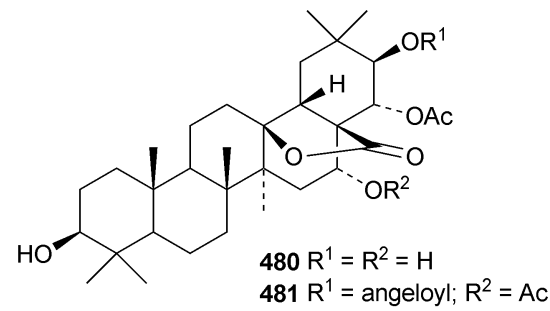

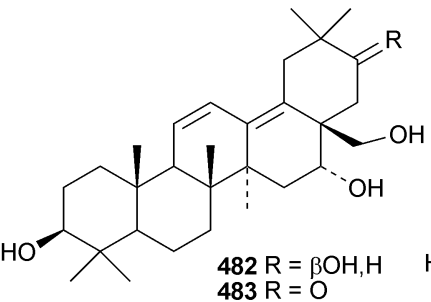<smiles>CC(=O)C1(C)CC[C@]2(C(=O)O)CCC[C@]3(C)C(=CCC4[C@@]5(C)CC[C@H](O)C(C)(C)C5CC[C@]43C)[C@@]12C</smiles>

Table 1

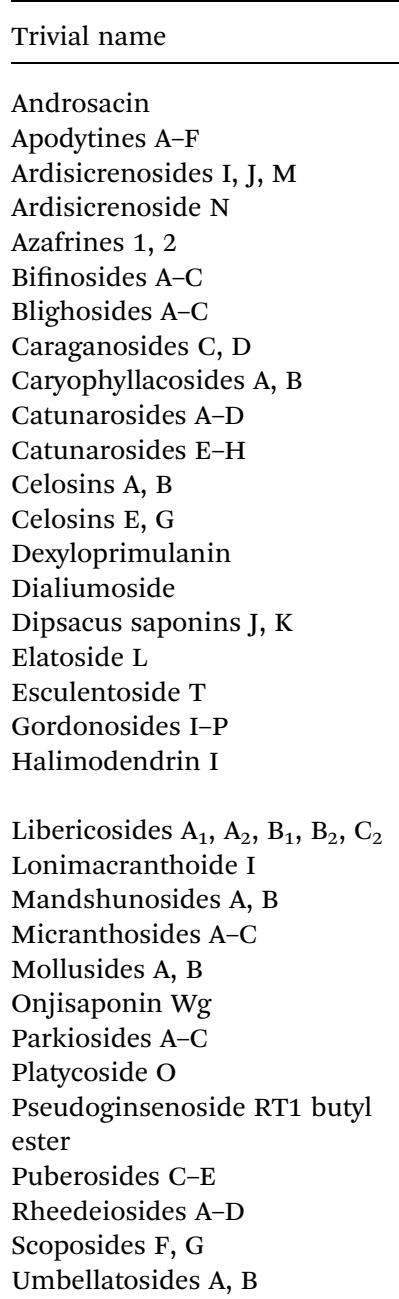

$\begin{array}{ll}\text { Plant species } & \text { Reference } \\ \text { Androsace integra } & 206 \\ \text { Apodytes dimidiata } & 207 \\ \text { Ardisia crenata } & 208 \\ \text { Ardisia crenata } & 209 \\ \text { Crocus sativus } & 210 \\ \text { Panax bipinnatifidus } & 211 \\ \text { Blighia sapida } & 212 \\ \text { Caragana microphylla } & 213 \\ \text { Gypsophila paniculata } & 214 \\ \text { Catunaregam spinosa } & 215 \\ \text { Catunaregam spinosa } & 216 \\ \text { Celosia argentea } & 217 \\ \text { Celosia argentea } & 218 \\ \text { Labisia pumila } & 219 \\ \text { Dialium excelsum } & 220 \\ \text { Dipsacus asper } & 221 \\ \text { Aralia elata } & 222 \\ \text { Phytolacca acinosa } & 223 \\ \text { Gordonia chrysandra } & 224 \\ \text { Halimodendron } & 225 \\ \text { halodendron } & \\ \text { Atroxima liberica } & 226 \\ \text { Lonicera macranthoides } & 227 \\ \text { Clematis mandshurica } & 228 \\ \text { Polygala micrantha } & 229 \\ \text { Albizia mollis } & 230 \\ \text { Polygala tenuifolia } & 231 \\ \text { Butyrospermum parkii } & 232 \\ \text { Platycodon grandiflorum } & 233 \\ \text { Panax japonicus var. major } & 96 \\ & \end{array}$

Glochidion puberum 234

Entada rheedei 235

Cephalaria spp. $\quad 236$

Hydrocotyle umbellata 237
Table 2

\begin{tabular}{|c|c|}
\hline Plant species & Reference \\
\hline Albizia inundata & 238 \\
\hline Anemone rivularis var. flore-minore & 239 \\
\hline Anemone taipaiensis & 240 \\
\hline Aralia elata & 241 \\
\hline Arenaria montana & 242 \\
\hline Bellis perennis & 243 \\
\hline Catunaregam spinosa & 244 \\
\hline Cylicodiscus gabunensis & 245 \\
\hline Dianthus superbus & 246 \\
\hline Erthrophleum fordii & 247 \\
\hline Ganoderma applanatum & 248 \\
\hline Gymnocladus chinensis & 249 \\
\hline Gypsophila perfoliata & 250 \\
\hline Juglans sinensiss & 189 \\
\hline Kalopanax pictus & 174 \\
\hline Lathyrus rattan & 251 \\
\hline Medicago polymorpha & 252 \\
\hline Microsechium helleri & 253 \\
\hline Nephelium lappaceum & 254,255 \\
\hline Panacis majoris & 256 \\
\hline Phytolacca americana & 257 \\
\hline Salsola imbricata & 258 \\
\hline Sanguisorba tenuifolia var. alba & 259 \\
\hline Symplocos caudata & 260 \\
\hline Symplocos lancifolia & 261 \\
\hline
\end{tabular}

478. ${ }^{201}$ Other oleanane saponins with new genins include

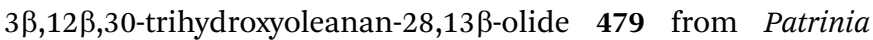
scabiosaefolia, ${ }^{202}$ the esters $\mathbf{4 8 0}$ and $\mathbf{4 8 1}$ from Maesa lanceolata ${ }^{203}$ oleana-11,13(18)-diene-3 $\beta, 16 \alpha, 21 \beta, 28$-tetrol 482 and the corresponding 21 ketone $\mathbf{4 8 3}$ from Bupleurum falcataum and Bupleurum rotundifolium, ${ }^{204}$ and coryternic acid $\mathbf{4 8 4}$ from Corydalis ternate. ${ }^{205}$ 


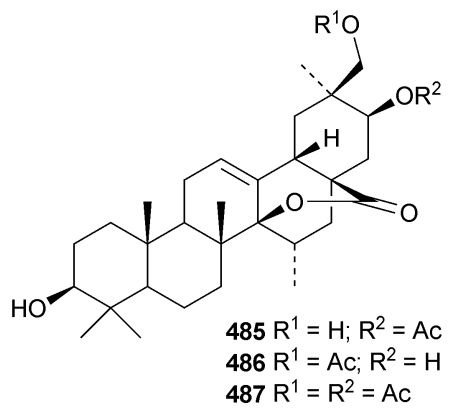<smiles>CC(=O)OCC1(C)C[C@H]2C3=CCC4[C@](C)(CCC5C(C)(C)[C@@H](O)CC[C@@]54C)C3=C(C)C[C@]2(C(=O)O)C[C@H]1OC(C)=O</smiles>

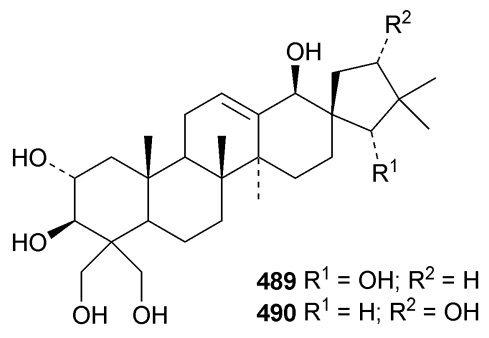

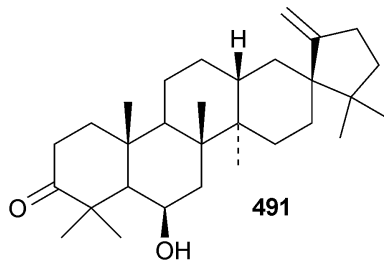<smiles>CC(=O)O[C@H]1CC[C@]2(C)C3=CC[C@@H]4[C@@H](CC[C@@]5(C)CC(C)(C)CC[C@]45C)[C@H]3CC=C2C1(C)C</smiles>

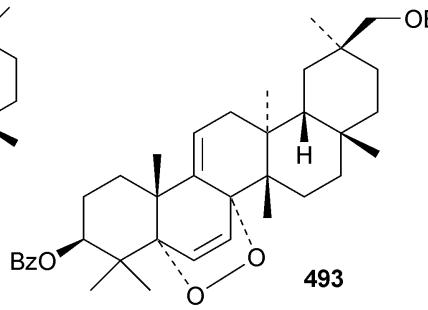<smiles>CC(=O)O[C@H]1CCC2=C(C(=O)C[C@@H]3[C@@H]4CC[C@](C)(CCC(C)(C)C)[C@]4(C)CC[C@@]23C)C1(C)C</smiles>

New oleanane saponins with known genins that have been assigned trivial names are listed in Table 1.

The sources of new oleanane saponins with known genins that have not been assigned trivial names are listed in Table 2.

Pachanosides C1, E1, F1 and G1 are pachanane saponins from Echinops macrogona with the genins 485-488, respectively. ${ }^{201}$ The structure of pachanol C 485 has been revised.

The rearranged oleanane derivatives phlomishexaols C 489 and D 490 have been found in the roots of Phlomis umbrosa. ${ }^{\mathbf{2 6 2 , 2 6 3}}$ The biosynthetic origin of the spirotriterpenoid cleistanone 491, from Cleistanthus indochinensis, is not clear from its structure. ${ }^{263}$ The rearranged oleanane derivative 492 has been claimed from Rhododendron campanulatum. ${ }^{\mathbf{2 6 4}}$ The stereochemistry of the methyl group at C-18 of 492 is unusual. The multiflorane endoperoxide dibenzoate 493 is a constituent of processed seeds of Trichosanthes kirilowii. ${ }^{265}$ 3ß-Acetoxyglutin-5(10)-en-6-one 494 has been found in roots of Scorzonera austriaca. ${ }^{266}$

New friedelane triterpenoids include $21 \alpha$-hydroxyfriedelane1,3-dione 495 from Salacia verrucosa ${ }^{267} 12 \alpha$-hydroxyfriedelane3,16-dione $\mathbf{4 9 6}^{\mathbf{2 6 8}}$ and 12 $\alpha, 29$-dihydroxyfriedelan-3-one $\mathbf{4 9 7 ^ { 2 6 9 }}$ from Maytenus gonoclada, 3 $\beta$-hydroxyfriedelane-7,12,22-trione 498 from Drypetes laciniata ${ }^{270}$ and $11 \alpha$-friedelan-3-one 499 from Myrica rubra. ${ }^{271}$ The norfriedelane derivative 3-O-methyl-6-oxopristimerol 500 is a constituent of Maytenus chubensis. ${ }^{272}$ Blepharodin 501, from Maytenus magellanica, is an adduct with a phenylpropanoid derivative. ${ }^{194}$

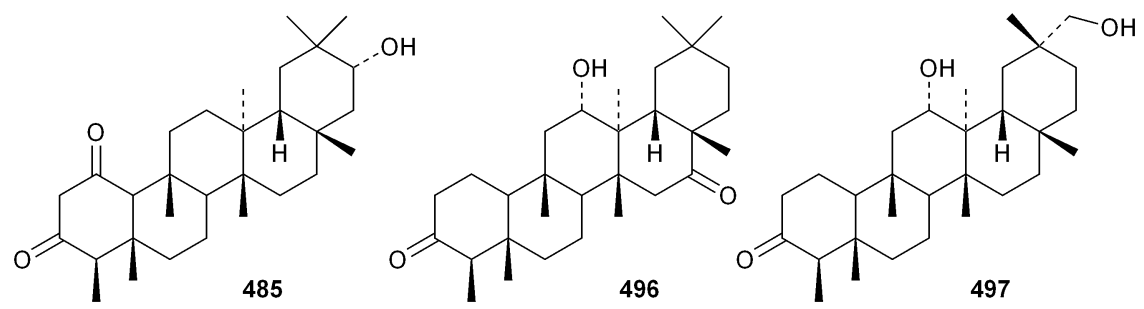


<smiles>COc1c(O)cc2c(c1C)C(=O)C=C1C23CCC2C1(C)CCC1(C)CCC(C)(C(C)=O)CC21C3</smiles><smiles>COc1cc(C2Oc3c(cc4c(c3C)C(=O)CC3C4(C)CCC4(C)C5CC(C)(C(C)=O)CCC5(C)CC34C)OC(COC(C)=O)C2C)cc(OC)c1O</smiles>

\section{The ursane group}

The 18,19-secoursane derivatives 502 and $\mathbf{5 0 3}$ have been isolated from Rosa laevigata together with 28-norursa-12,17-diene$2 \alpha, 3 \beta, 23$-triol 504 and the arabinoside 505 whose genin has an unusual $19 \alpha$-stereochemistry. ${ }^{188}$ The related 18,19-secoursane derivative 506 has been reported from leaves of Diospyros $k a k i{ }^{273}$ Atriplicaide A $\mathbf{5 0 7}$ is an unusual $\mathrm{N}$-acetyl lactam from Zygophyllum eurypterum where it is found with atriplicaide $\mathrm{B}$ 508 which is $3 \beta, 24$ dihydroxyursan-28,13-olide. ${ }^{274}$ Related 28,13olides 509 and $\mathbf{5 1 0}$ have been isolated from Isodon coets $a^{275}$ and Schefflera heptaphylla, ${ }^{276}$ respectively. Proceraursenolide 511, from the roots of Calatropis procera, is claimed to be $18 \alpha H$-urs-12-en-25,3 $\beta$-olide. ${ }^{277}$ Other new simple ursane derivatives include cordinoic acid $\mathbf{5 1 2}$ from Cordia latifolia, ${ }^{278}$ urs-12-ene-3 $\alpha, 23$-diol 513 from Salvia miltiorrhiza, ${ }^{\mathbf{1 8 5}} 18 \alpha \mathrm{H}^{-}$ ursene-3 $\beta, 20 \beta$-diol $\mathbf{5 1 4}$ from Boswellia carterii, ${ }^{\mathbf{1 1 6}}$ $1 \alpha, 2 \alpha, 3 \beta, 16 \alpha, 19 \alpha, 20 \beta$-hexahydroxyurs-12-en-28-oic acid $\mathbf{5 1 5}$ from Pedicularis kansuensis, ${ }^{279}$ glutinolic acid 516 from Rehmannia glutinosa, ${ }^{280}$ and 3 $3,7 \beta, 24$-trihydroxyurs-12-en-28-oic acid $\mathbf{5 1 7}$ from Saurauja roxburghii. ${ }^{281}$ New ursane ester derivatives include conrauidienol $\mathbf{5 1 8}$ from Ficus conraui, ${ }^{282}$ 3ß-acetoxyursa-11,13(18)-dien-28-oic acid 519 from Eucalyptus camaldulensis, ${ }^{283} 3$-O-acetyluncaric acid $\mathbf{5 2 0}$ from Radermachera

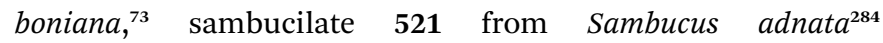
and the palmitate esters $\mathbf{5 2 2}$ and $\mathbf{5 2 3}$ from Viburnum betulifolium. $^{285}$

Clethroidoside $\mathrm{H}$, from Lysimchia clethroides, is an ursane saponin with the new genin ursa-9(11),12-diene-2 $\alpha, 3 \beta, 21 \beta, 30$ tetrol 524. ${ }^{196}$ The 18,19-secoursane derivative 525 is the genin of dunnianolactones A-C from Ilex dunniana. ${ }^{286}$ A saponin given the duplicate name ilexsaponin $\mathrm{C}$, from Ilex pubescens, has the

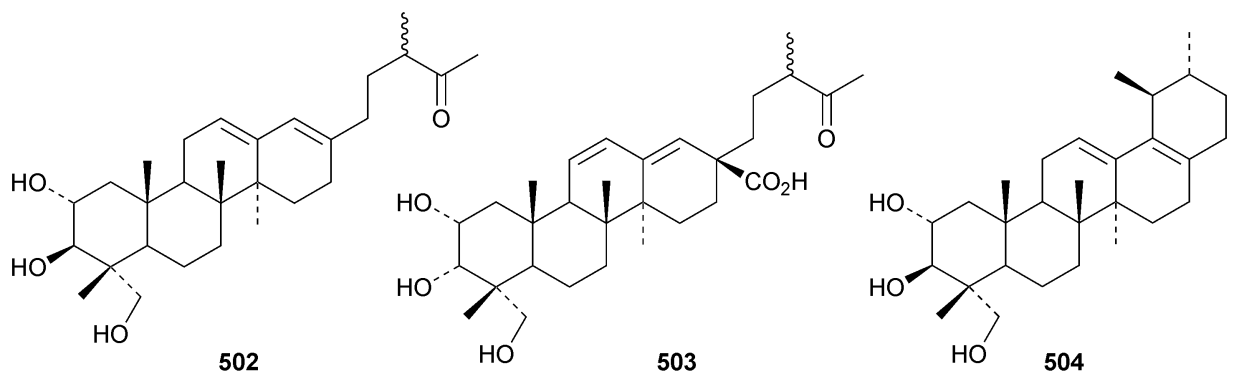

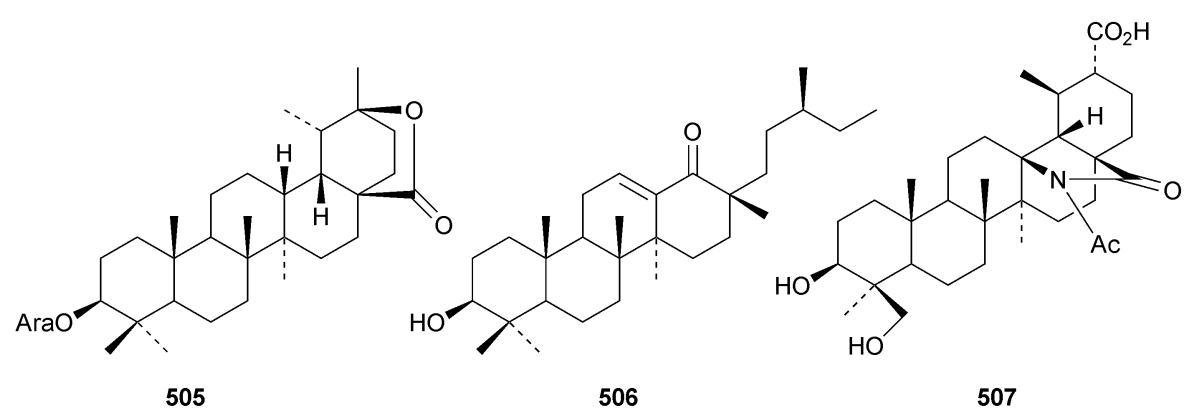




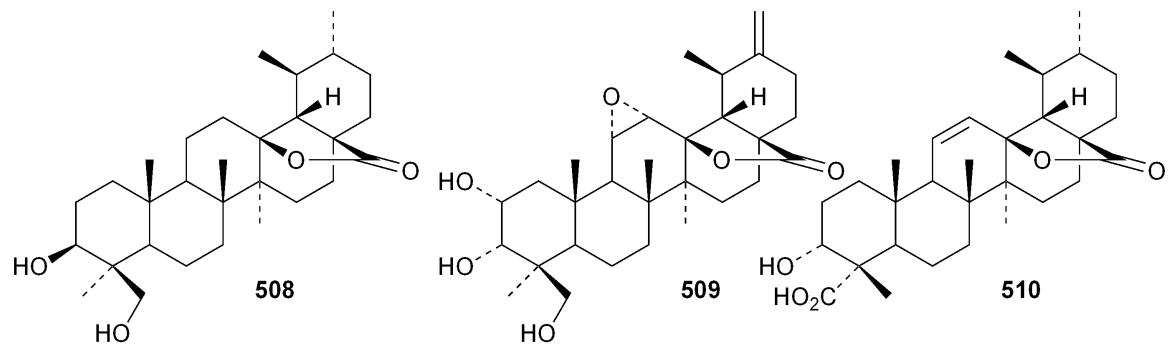

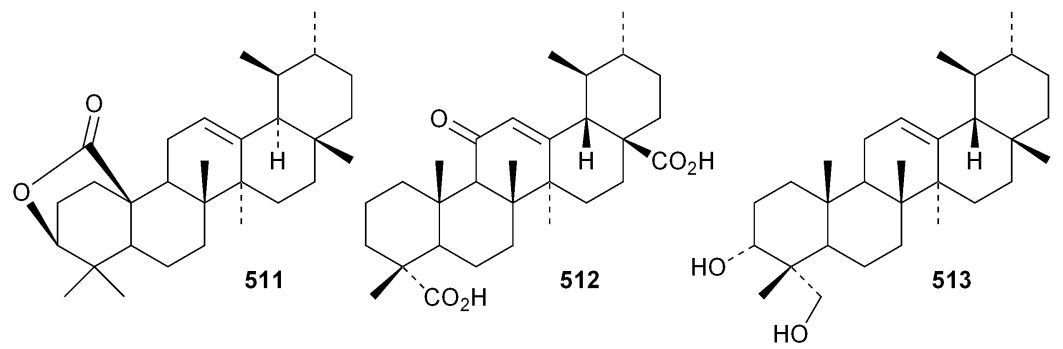

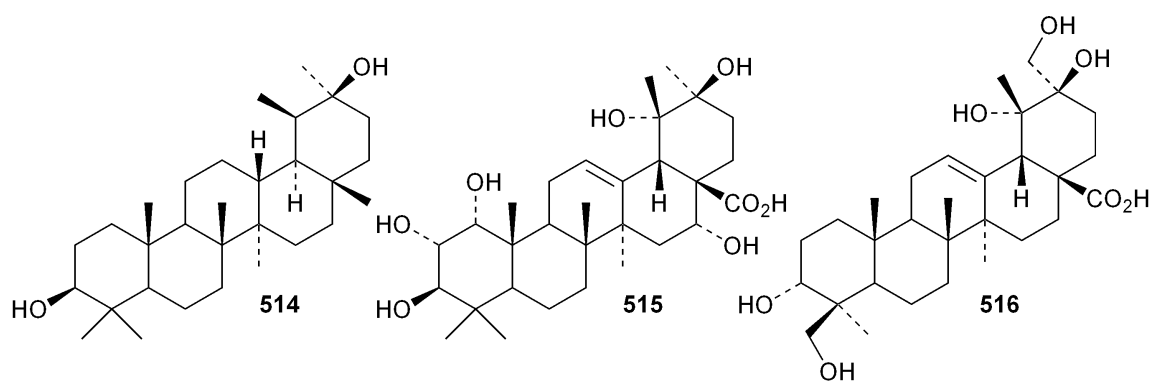

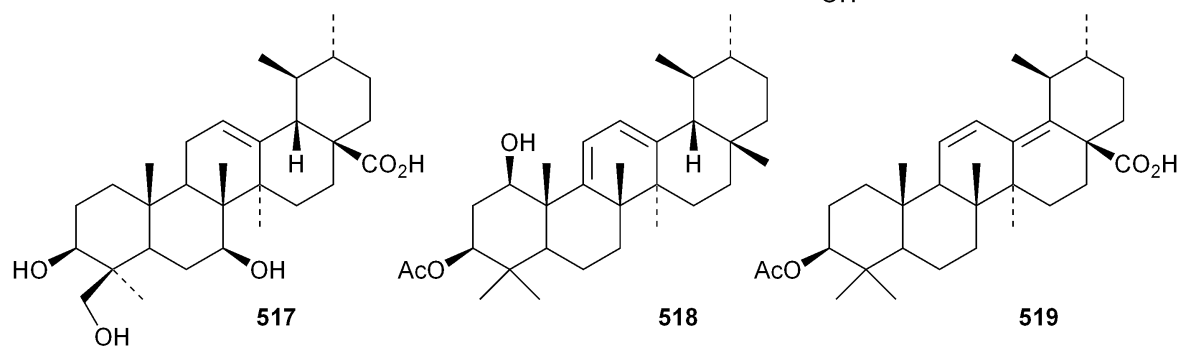

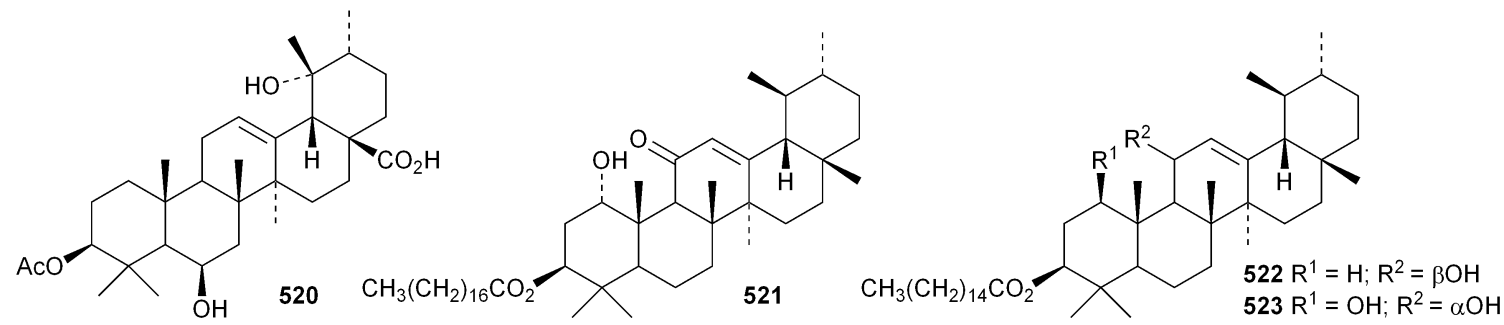

new genin 28-norursa-12,17-dien-2 $\beta$-ol 526. ${ }^{287}$ Other unnamed ursane saponins with the new genins include $3 \beta, 23$-dihydroxyurs-12-en-28-oic acid $\mathbf{5 2 7}$ from Jugalans sinensis, ${ }^{\mathbf{1 8 9}}$ and 2,3 $\beta, 16 \alpha, 23$-tetrahydroxyurs-12-en-28-oic acid $\mathbf{5 2 8}$ from Lathyrus aphaca. ${ }^{251}$

Ursane saponins with known genins include asiaticoside $\mathrm{G}$ from Centella asiatica, ${ }^{288}$ clethric acid 28-O- $\beta$-D-glucopyransyl ester and mussaendoside $\mathrm{T}$ from Anthocephalus chinensis, ${ }^{289}$ ilekudinchosides $\mathrm{A}-\mathrm{D}^{290}$ and $\mathrm{W}^{291}$ from Ilex kudincha, symplocosins A and B from Symplocos cochinchinensis var. philippensis ${ }^{292}$ zygophylloside $\mathrm{S}$ from Zygophyllum coccineum ${ }^{293}$ and unnamed saponins from Ilex chamaedryfolia, ${ }^{294}$ Juglans sinensis, ${ }^{\mathbf{1 8 9}}$ Sanguisorba tenuifolia var. alba ${ }^{259}$ and Symplocos lancifolia. $^{261}$

19ß-Hydroxy-3,4-seco-4(23),20(30)-taraxastadien-3-oic acid 529 has been isolated from buds of Betula pendula. ${ }^{173}$ The 


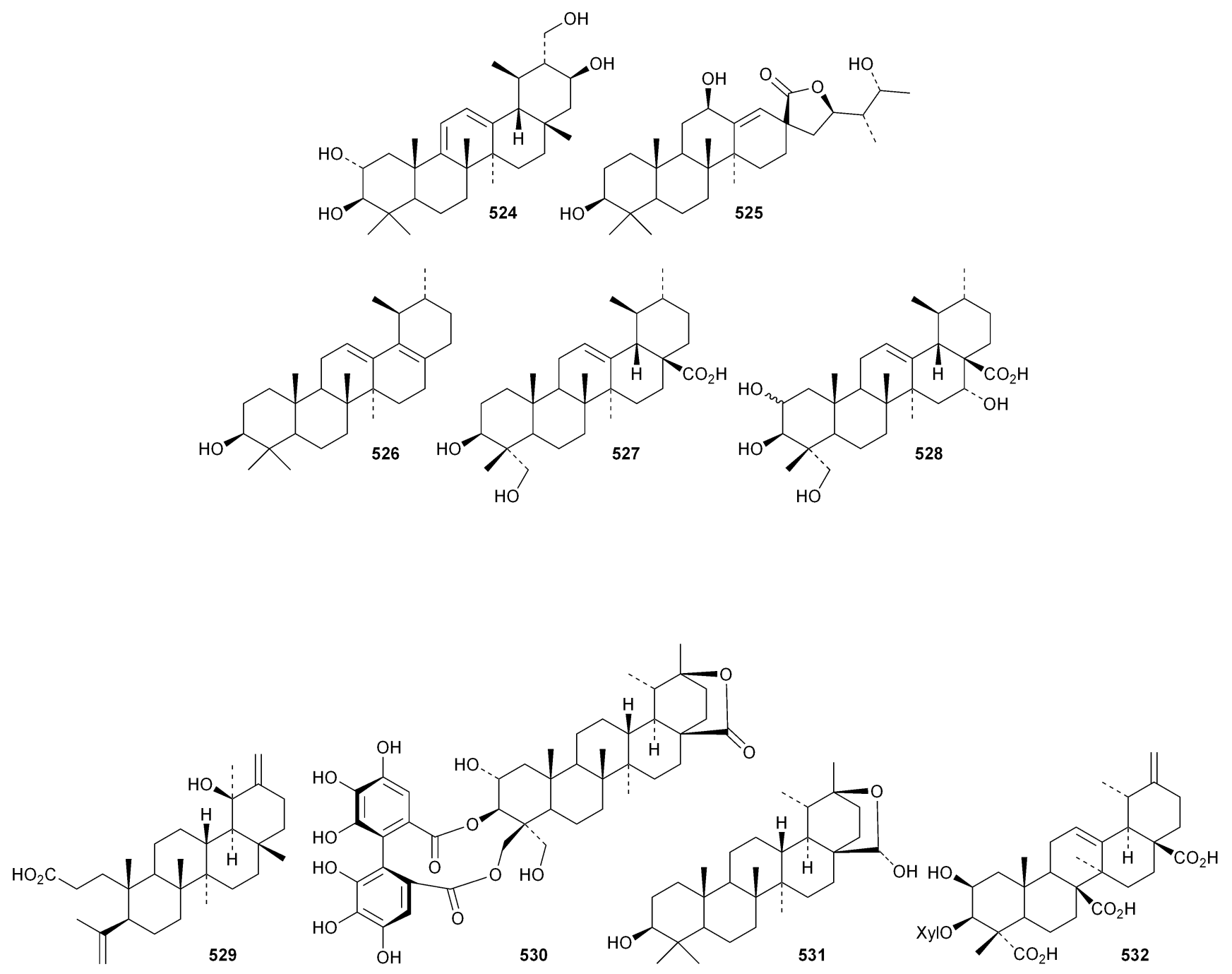

tannin ester $\mathbf{5 3 0}$ of $2 \alpha, 3 \beta, 23,24$-tetrahydroxytaraxastan-28,20 $\beta$ olide is a constituent of leaves of Castanopsis fissa. ${ }^{\mathbf{1 9 2}}$ The taraxastane hemiacetal $\mathbf{5 3 1}$ has been found in Geum japonicum. ${ }^{295}$ Celosin F 532 appears to be a taraxastane xyloside from Celosia argentea. $^{218}$

\section{The hopane group}

The current knowledge of squalene-hopene cyclases has been reviewed. ${ }^{296}$ The unusual 9,25-cyclo-29-propylhopan-31-ol 533 and $3 \beta$-hydroxy-29-propylhopan-31-one $\mathbf{5 3 4}$ have been identified in

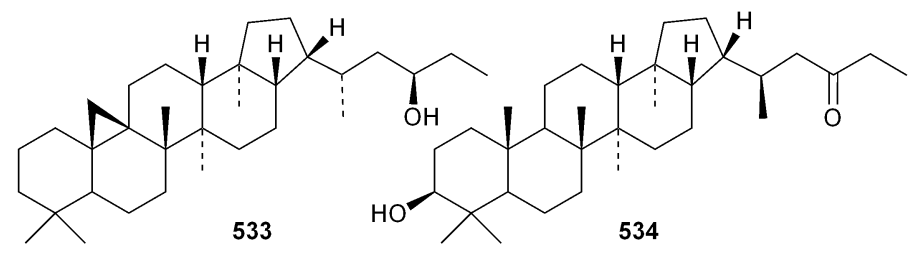

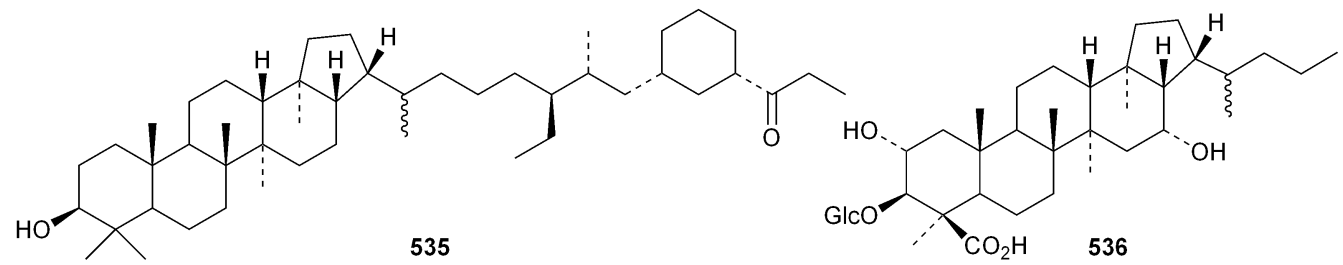




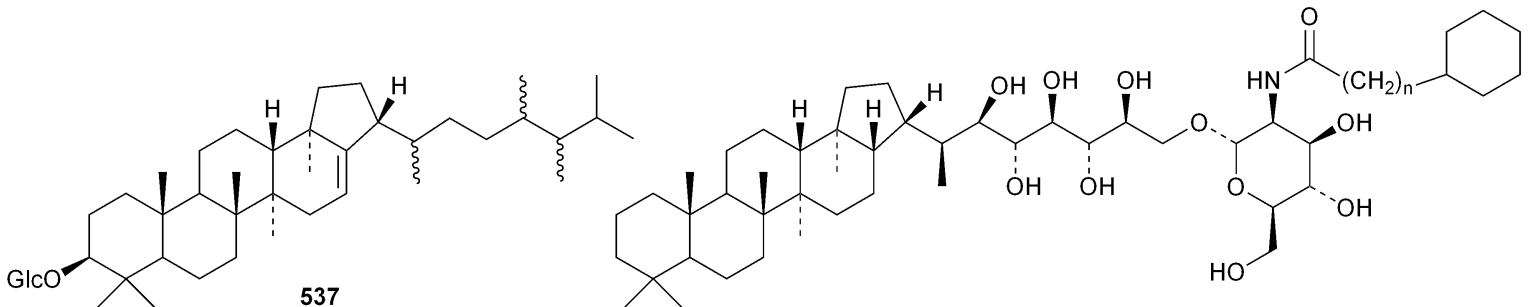

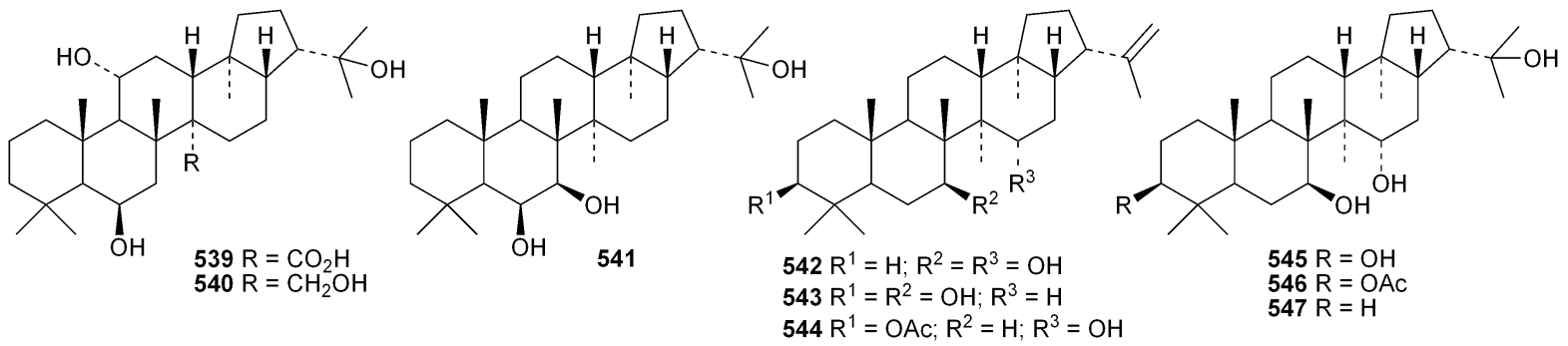

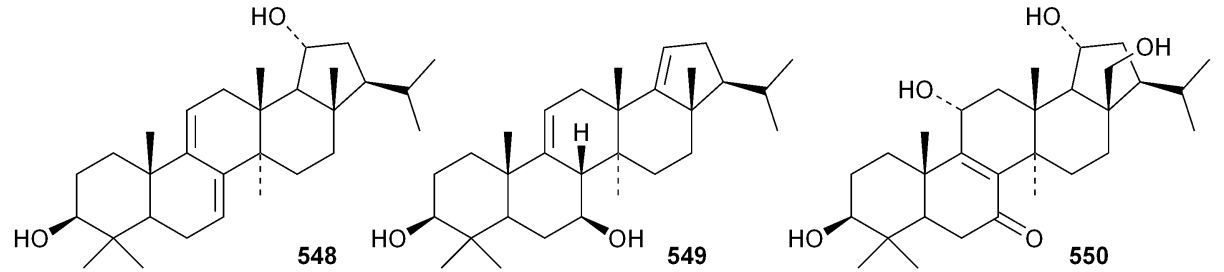

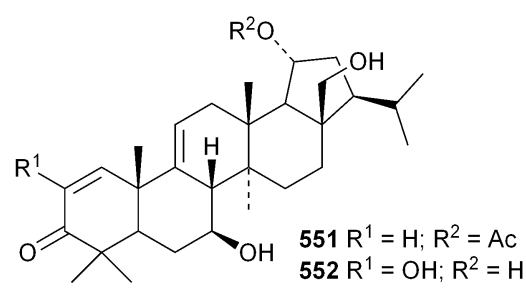<smiles>[R17][R15]</smiles><smiles>[R2]O[C@H]1C[C@H]2C(C)(C)C([Z7])CC[C@]2(C)C2=CC[C@](C)(C(C)CO)[C@@](C)(CC)[C@]21C</smiles>
$555 \mathrm{R}^{1}=\mathrm{OGIC}(1->2) \mathrm{Glc} ; \mathrm{R}^{2}=\mathrm{H} ; \mathrm{R}^{3}=\mathrm{CH}_{2} \mathrm{OH}$ $556 \mathrm{R}^{1}=\mathrm{OGlc}(1->6) \mathrm{Glc} ; \mathrm{R}^{2}=\mathrm{H} ; \mathrm{R}^{3}=\mathrm{CH}_{2} \mathrm{OAc}$ $557 \mathrm{R}^{1}=\mathrm{OGIC}(1->6) \mathrm{Glc} ; \mathrm{R}^{2}=\mathrm{H} ; \mathrm{R}^{3}=\mathrm{CHO}$

Celestris australis. ${ }^{176}$ The same group claim that the cyclohexylhopane derivative $\mathbf{5 3 5}$ is also found in Celestris australis ${ }^{297}$ and that the 29-ethylhopane derivative 536 and 32,33,34-trimethylbacteriohopan-3 $\beta$-yl $\beta$-D-glucopyranoside 537 are constituents of Symplocos paniculata. ${ }^{177}$ Several $N$-acylated bacteriohopanehexol mannosamine derivatives $\mathbf{5 3 8}$ have been identified in the thermophilic bacterium Alicyclobacillus acidoterrestris. ${ }^{298}$ The simple hopane derivatives $\mathbf{5 3 9 - 5 4 1}{ }^{299}$ and $\mathbf{5 4 2 - 5 4 7 ^ { 3 8 }}$ are metabolites of the entomopathogenic fungi Conoideocrella tenuis and Hypocrella sp. BCC 14524, respectively.

Twelve arborinane triterpenoids have been isolated from Rubia yunnanensis including rubiyunnanols A-C 548-550, rubiarbonone E 19-acetate 551, 2-hydroxyrubiarbonone E 552, 19,28-didehydroxyrubiarbonol A 553, rubiarbonol A 7-acetate 554, the

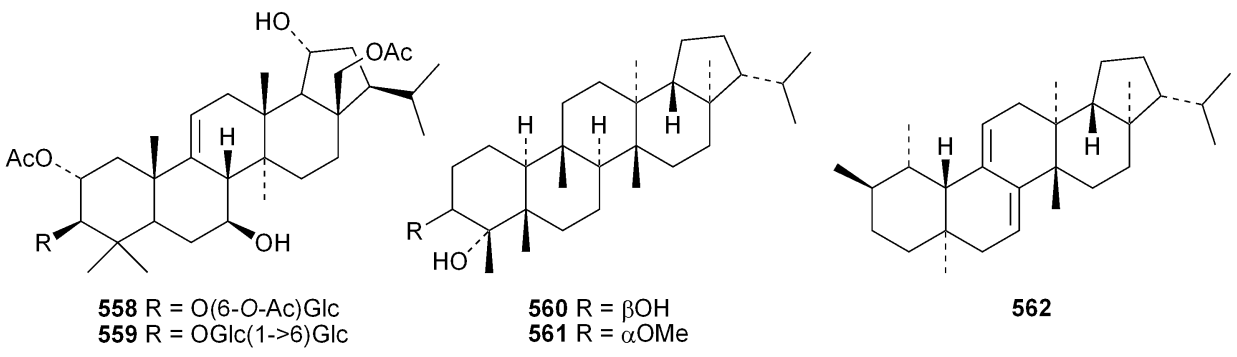


rubiarbonol A glycoside 555, rubiarboside G 28-acetate 556, rubiarboside G 28-aldehyde 557, $2 \alpha$-acetoxyrubiarboside 28acetate 558 and the rubianol E glycoside 559. ${ }^{300}$ Adiantum capillusveneris is the source of filicane- $3 \beta, 4 \alpha$-diol 560 and the corresponding $3 \alpha$-methyl ether 561. ${ }^{301}$ Canarene 562 is an unusual rearranged filicane derivative from Canarium schweinfurthii. ${ }^{302}$ The structure of canarene $\mathbf{5 6 2}$ was confirmed by X-ray analysis and a biosynthetic scheme for its formation has been proposed.

\section{Miscellaneous compounds}

Phyteumosides A and B, from Phyteuma orbiculare, have as aglycones the partially cyclised onocerane triterpenoid 563 and the polypodane derivative 564 , respectively. ${ }^{303}$ The structures of the aglycones 563 and 564 were established by X-ray analysis. Lansium domesticum is the source of the onocerane derivatives lamesticumin A 565 and the corresponding ethyl ester 566, lamesticumins B-E 567-570, the 3-ethyl ester of lansic acid 571 and ethyl lansiolate $\mathbf{5 7 2}$ together the polypodane derivative lamesticumin $\mathrm{F} \mathbf{5 7 3 .} .^{\mathbf{3 0 4}}$ Other onocerane derivatives include cupacinoxepin 574, from Cupania cinerea ${ }^{\mathbf{3 0 5}}$ and kokosanolide B $\mathbf{5 7 5}$ and onocera-7,14-diene-3,21-dione $\mathbf{5 7 6}$ from Lansium domesticum cv. Kokossan. ${ }^{126}$

The isomalabaricane triterpenoids stelliferins J-N 577-581 are constituents of the sponge Rhabdastrella $c f$. globostellata. ${ }^{306}$ Stelliferins L-N 579-581 have a cyclised side-chain similar to rhabdastins D-G. A sponge of the genus Lipastrotethya is the source of pouosides $\mathrm{F}-\mathrm{I}$ 582-585 and pouogenins $\mathrm{A}-\mathrm{E}$

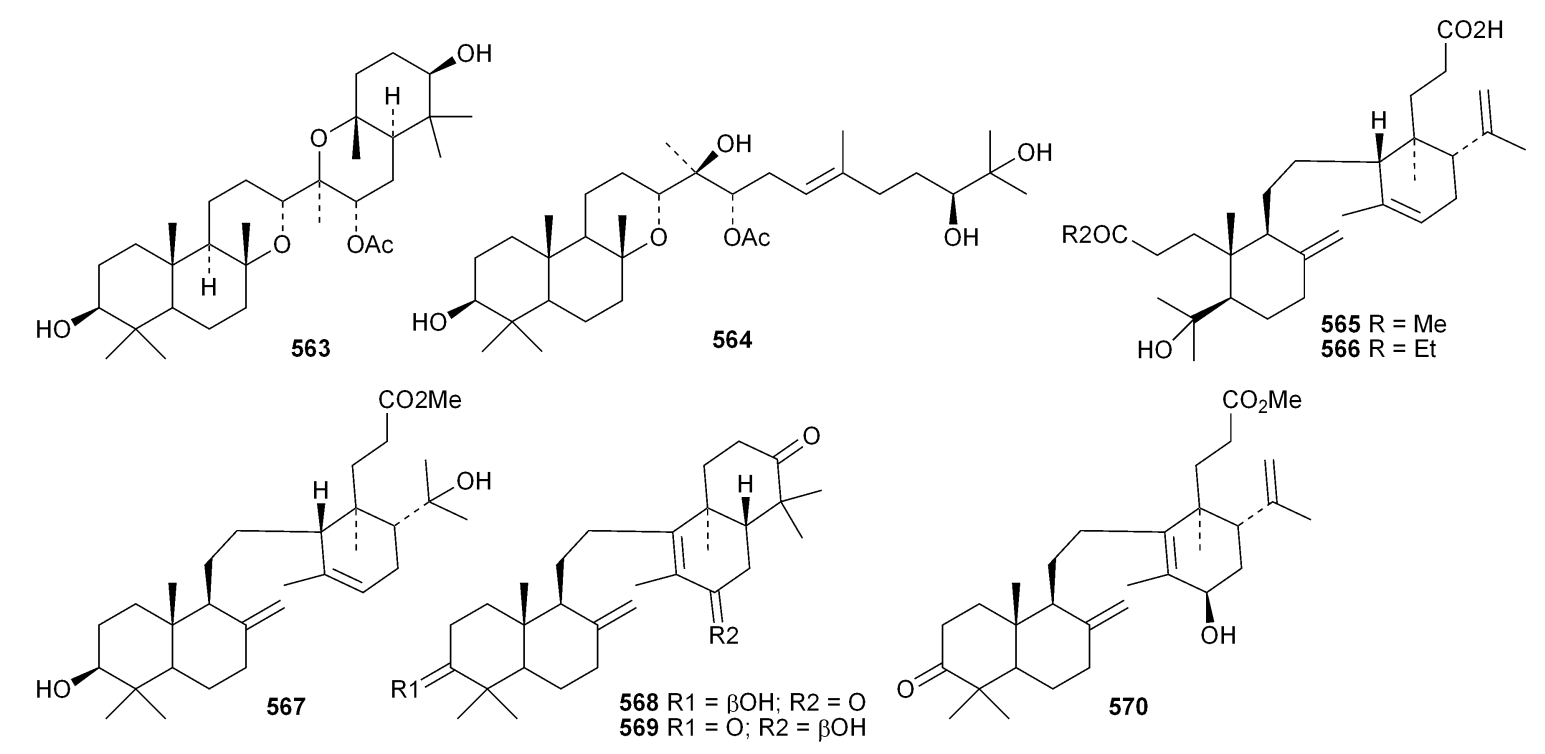

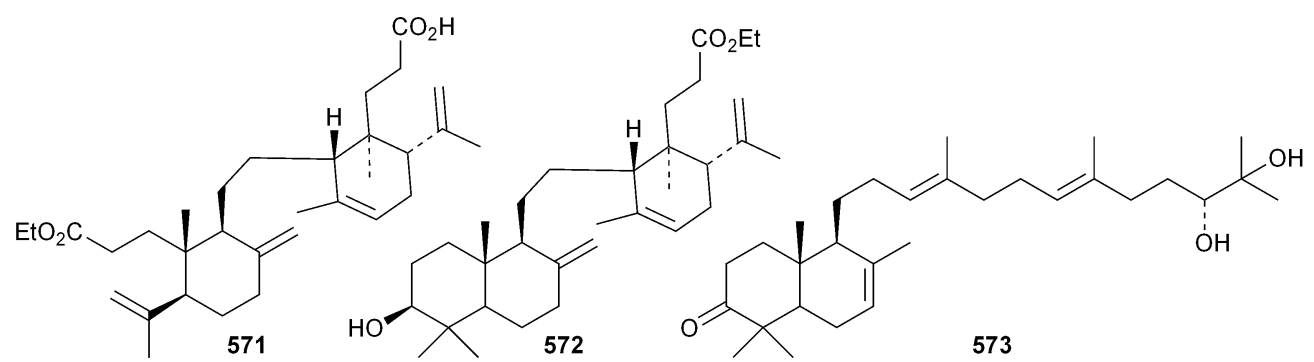

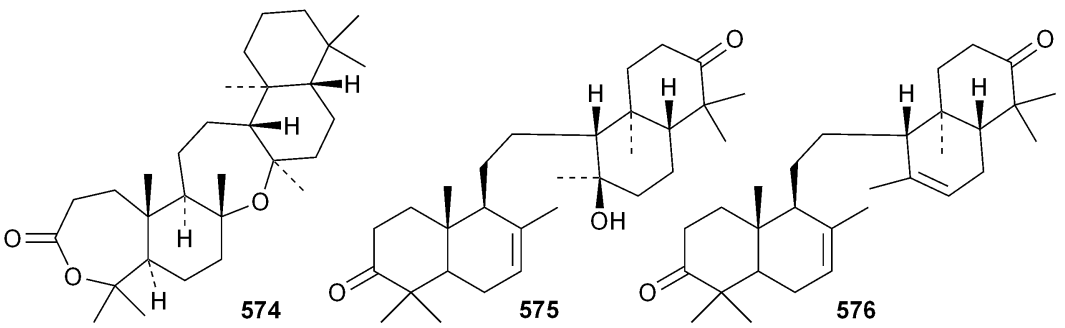



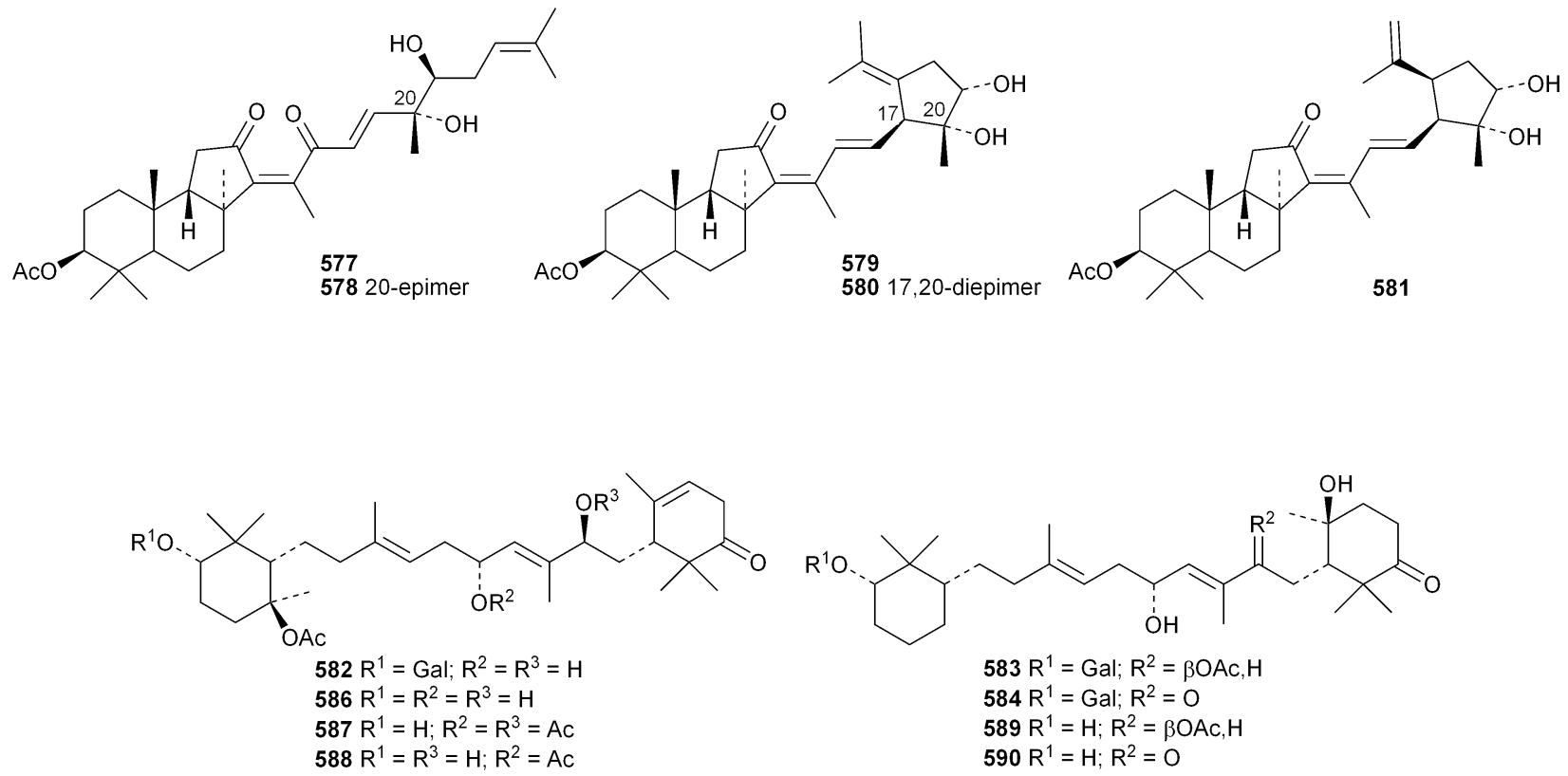<smiles>C=C1CCC(OCCO)C(C)(C)[C@@H]1CC/C(C)=C/C[C@@H](/C=C(\C)[C@H](CC1C(C)=CCC(=O)C1(C)C)OC(C)=O)OC(C)=O</smiles><smiles>CC(C)=CCC/C(C)=C/[C@H](O)C/C(C)=C/CC[C@@]1(C)[C@@H](C)CC/C(=C(/C)C=O)[C@@H]1CCCO</smiles><smiles>[R16][R16]#[Z10]OCC</smiles>

586-590. ${ }^{307}$ Three iridal triterpenoids 591-593 have been isolated from Iris delavayi..$^{\mathbf{3 0}}$

\section{References}

1 M. B. Sporn, K. T. Liby, M. M. Yore, L. Fu, J. M. Lopchuk and G. W. Gribble, J. Nat. Prod., 2011, 74, 537-545.

2 A. Braca, P. F. Dal, S. Marzocco, G. Autore, A. Vassallo and T. N. De, Curr. Drug Targets, 2011, 12, 302-321.

3 Z. Meng, N.-g. Li, L. Zhang and A.-w. Ding, Zhonghua Zhongyiyao Xuekan, 2011, 29, 1152-1154.

4 Y. Zhang, X. Zhang and G. Chen, Hainan Shifan Daxue Xuebao, Ziran Kexueban, 2011, 24, 92-95.

5 M. E. Juan and J. M. Planas, Bioact. Foods Extr., 2011, 403413.

6 A. Bishayee, S. Ahmed, N. Brankov and M. Perloff, Front. Biosci., 2011, 16, 980-996.

7 B. K. Cassels and M. Asencio, Phytochem. Rev., 2011, 10, 545-564.
8 A. Gonzalez-Coloma, C. Lopez-Balboa, O. Santana, M. Reina and B. M. Fraga, Phytochem. Rev., 2011, 10, 245-260.

9 H. Sheng and H. Sun, Nat. Prod. Rep., 2011, 28, 543593.

10 Y.-X. Sun, J.-C. Liu and D.-Y. Liu, Pharmazie, 2011, 66, 813821.

11 X.-s. Zhang and X.-m. Hu, Anhui Nongye Kexue, 2011, 39, 817-818, 825.

12 H. Hussain, J. Hussain, A. Al-Harrasi and Z. K. Shinwari, Pak. J. Bot., 2011, 43, 51-62.

13 L. F. Barbosa, R. Braz-Filho and I. J. C. Vieira, Chem. Biodiversity, 2011, 8, 2163-2178.

14 T. P. Kukina and E. N. Shmidt, Khim. Interesakh Ustoich. Razvit., 2011, 19, 655-659.

15 Q.-G. Tan and X.-D. Luo, Chem. Rev., 2011, 111, 7437-7522.

16 A. Osbourn, R. J. M. Goss and R. A. Field, Nat. Prod. Rep., 2011, 28, 1261-1268.

17 J. M. Augustin, V. Kuzina, S. B. Andersen and S. Bak, Phytochemistry, 2011, 72, 435-457. 
18 E. Lambert, A. Faizal and D. Geelen, Appl. Biochem. Biotechnol., 2011, 164, 220-237.

19 P. Zhao, D.-F. Gao, M. Xu, Z.-G. Shi, D. Wang, C.-R. Yang and Y.-J. Zhang, Chem. Biodiversity, 2011, 8, 1931-1942.

$20 \mathrm{~J}$. Ni, M. Chen and Y. Lin, Zhongyaocai, 2011, 34, 317-319.

21 X. Xia, C. Zheng, C. Feng and C. Xia, Chaye Kexue, 2011, 31, 391-398.

22 S. Boettger and M. F. Melzig, Phytochem. Lett., 2011, 4, 5968.

23 F. Cen-Pacheco, J. A. Villa-Pulgarin, F. Mollinedo, M. Norte, A. H. Daranas and J. J. Fernández, Eur. J. Med. Chem., 2011, 46, 3302-3308.

24 F. Cen-Pacheco, J. A. Villa-Pulgarin, F. Mollinedo, M. N. Martin, J. J. Fernández and A. H. Daranas, Mar. Drugs, 2011, 9, 2220-2235.

25 R. C. Jadulco, M. Koch, W. R. M. Van, C. Pond, O. G. Gideon, T. Matainaho, P. Piskaut and L. R. Barrows, Planta Med., 2011, 77, 1651-1654.

26 M. Spanova and G. Daum, Eur. J. Lipid Sci. Technol., 2011, 113, 1299-1320.

27 F.-Y. Meng, J.-X. Sun, X. Li, H.-F. Pi, P. Zhang and H.-L. Ruan, Helv. Chim. Acta, 2011, 94, 1778-1785.

28 S. Dall'Acqua, P. Minesso, S. Comai, B. B. Shrestha, M. B. Gewali, P. K. Jha and G. Innocenti, Nat. Prod. Commun., 2011, 6, 793-798.

29 D.-W. Ou-Yang, L. Wu, Y.-L. Li, P.-M. Yang, D.-Y. Kong, X.-W. Yang and W.-D. Zhang, Phytochemistry, 2011, 72, 2197-2204.

30 A. Tsopmo and P. Kamnaing, Phytochem. Lett., 2011, 4, 218221.

31 T.-G. Cai and Y. Cai, Chem. Biodiversity, 2011, 8, 2135-2143. 32 X.-Q. Zhang, F. C. F. Ip, D.-M. Zhang, L.-X. Chen, W. Zhang, Y.-L. Li, N. Y. Ip and W.-C. Ye, Nat. Prod. Res., 2011, 25, 1607-1613.

33 I. S. Lee, B. R. Ahn, J. S. Choi, M. Hattori, B. S. Min and K. H. Bae, Bioorg. Med. Chem. Lett., 2011, 21, 6603-6607.

34 S. Joseph, K. K. Janardhanan, V. George and S. Baby, Phytochem. Lett., 2011, 4, 386-388.

35 J.-L. Rios, Planta Med., 2011, 77, 681-691.

36 M. R. Camargo and R. Kaneno, Annu. Rev. Biomed. Eng., 2011, 13, 1-8.

37 X.-W. Shi, X.-J. Li, J.-M. Gao and X.-C. Zhang, Chem. Biodiversity, 2011, 8, 1864-1870.

38 M. Isaka, P. Chinthanom, M. Sappan, R. Chanthaket, J. J. Luangsa-ard, S. Prabpai and P. Kongsaeree, J. Nat. Prod., 2011, 74, 2143-2150.

39 T. Lin, X. Lin, C.-H. Lu and Y.-M. Shen, Helv. Chim. Acta, 2011, 94, 301-305.

40 R. Tanaka, M. Toyoshima and T. Yamada, Phytochem. Lett., 2011, 4, 328-332.

41 A. S. Antonov, A. I. Kalinovsky, P. S. Dmitrenok, V. I. Kalinin, V. A. Stonik, E. Mollo and G. Cimino, Carbohydr. Res., 2011, 346, 2182-2192.

42 M. Ono, D. Toyohisa, T. Morishita, H. Horita, S. Yasuda, Y. Nishida, T. Tanaka, M. Okawa, J. Kinjo, H. Yoshimitsu and T. Nohara, Chem. Pharm. Bull., 2011, 59, 1348-1354.

43 Z. Jabbar and M. Ali, Int. Res. J. Pharm., 2011, 2(7), 141-144.
44 A. S. Silchenko, A. I. Kalinovsky, S. A. Avilov, P. V. Andryjaschenko, P. S. Dmitrenok, E. A. Yurchenko and V. I. Kalinin, Nat. Prod. Commun., 2011, 6, 10751082.

45 V. P. Careaga, C. Muniain and M. S. Maier, Chem. Biodiversity, 2011, 8, 467-475.

46 A. S. Antonov, S. A. Avilov, A. I. Kalinovsky, P. S. Dmitrenok, V. I. Kalinin, S. Taboada, M. Ballesteros and C. Avila, Nat. Prod. Res., 2011, 25, 1324-1333.

47 Y.-B. Xue, J.-H. Yang, X.-N. Li, X. Du, J.-X. Pu, W.-L. Xiao, J. Su, W. Zhao, Y. Li and H.-D. Sun, Org. Lett., 2011, 13, 1564-1567.

48 Y.-C. Lin, I. W. Lo, S.-Y. Chen, P.-H. Lin, C.-T. Chien, S.-y. Chang and Y.-C. Shen, Org. Lett., 2011, 13, 446-449.

49 F.-Y. Meng, J.-X. Sun, X. Li, H.-Y. Yu, S.-M. Li and H.-L. Ruan, Org. Lett., 2011, 13, 1502-1505.

50 J.-R. Wang, Z.-B. Zhao and Y.-W. Guo, J. Asian Nat. Prod. Res., 2011, 13, 551-555.

51 Y. Jiang, G.-Z. Yang, Y. Chen, M.-C. Liao, X.-M. Liu, S. Chen, L. Liu and X.-X. Lei, Helv. Chim. Acta, 2011, 94, 491-496.

52 G.-Y. Yang, Y.-K. Li, X.-J. Zhang, X.-N. Li, L.-M. Yang, Y.-M. Shi, W.-L. Xiao, Y.-T. Zheng and H.-D. Sun, Nat. Prod. Bioprospect., 2011, 1, 33-36.

53 J.-M. Tan, Y.-H. Qiu, X.-Q. Tan and C.-H. Tan, Helv. Chim. Acta, 2011, 94, 1697-1702.

54 W.-J. He, H.-B. Chu, Y.-M. Zhang, H.-J. Han, H. Yan, G.-Z. Zeng, Z.-H. Fu, O. Olubanke and N.-H. Tan, Planta Med., 2011, 77, 1924-1931.

55 H.-X. Kuang, Q.-H. Wang, B.-Y. Yang, Z.-B. Wang, Y. Okada and T. Okuyama, Helv. Chim. Acta, 2011, 94, 2239-2247.

56 M. K. Jamroz, M. H. Jamroz, J. C. Dobrowolski, J. A. Glinski, M. H. Davey and I. Wawer, Spectrochim. Acta, Part A, 2011, 78A, 107-112.

57 Y. Ippongi, T. Ohtsuki, K. Toume, M. A. Arai, Y. Yamamoto and M. Ishibashi, Chem. Pharm. Bull., 2011, 59, 279-281.

58 Y. Kashiwada, K. Nishimura, S.-i. Kurimoto and Y. Takaishi, Bioorg. Med. Chem., 2011, 19, 2790-2796.

59 T. Nuanyai, R. Sappapan, T. Vilaivan and K. Pudhom, Phytochem. Lett., 2011, 4, 26-29.

60 Y. Shu, L. Cheng and P. Yang, Zhongcaoyao, 2011, 42, 805813.

61 H.-T. Kuo, C.-F. Peng, H.-Y. Huang, C.-H. Lin, I.-S. Chen and I.-L. Tsai, Planta Med., 2011, 77, 736-741.

62 T. Nuanyai, R. Sappapan, T. Vilaivan and K. Pudhom, Chem. Pharm. Bull., 2011, 59, 385-387.

63 R. Sun, H.-C. Song, C.-R. Wang, K.-Z. Shen, Y.-B. Xu, Y.-X. Gao, Y.-G. Chen and J.-Y. Dong, Bioorg. Med. Chem. Lett., 2011, 21, 961-965.

64 Y. Nian, X.-M. Zhang, Y. Li, Y.-Y. Wang, J.-C. Chen, L. Lu, L. Zhou and M.-H. Qiu, Phytochemistry, 2011, 72, 14731481.

65 D.-S. Li, Y. Nian, Y. Sun and M.-H. Qiu, Helv. Chim. Acta, 2011, 94, 632-638.

66 M. Nishida and H. Yoshimitsu, Chem. Pharm. Bull., 2011, 59, 1243-1249.

67 Z. Zhao, K. Matsunami, H. Otsuka, T. Shinzato and Y. Takeda, Chem. Pharm. Bull., 2011, 59, 902-905. 
68 H. D. Nguyen, B. T. D. Trinh, Q. N. Tran, H. D. Nguyen, H. D. Pham, P. E. Hansen, F. Duus, J. D. Connolly and L.-H. D. Nguyen, Phytochemistry, 2011, 72, 290-295.

69 K. Toume, T. Nakazawa, T. Ohtsuki, M. A. Arai, T. Koyano, T. Kowithayakorn and M. Ishibashi, J. Nat. Prod., 2011, 74, 249-255.

70 S. Jan, A. Abbaskhan, S. G. Musharraf, S. A. Sattar, Samreen, S. I. Resayes, Z. A. Al-Othman, A. M. Al-Majid, R. Attaur and M. I. Choudhary, Planta Med., 2011, 77, 1829-1834.

71 H. N. Nguyen, P. V. Kiem, N. K. Ban, P. T. Nguyen, X. N. Nguyen, X. C. Nguyen, C. Tistaert, B. Dejaegher, H. Y. Vander, J. Quetin-Leclercq, D. T. Thao and M. C. Van, Phytochem. Lett., 2011, 4, 348-352.

72 Y. Qiang, J.-M. Ni, Y.-B. Shi, X.-Y. Zhang, X. Yang and S.-T. Li, J. Chem. Res., 2011, 35, 664-665.

73 N. B. Truong, C. V. Pham, H. T. M. Doan, H. V. Nguyen, C. M. Nguyen, H. T. Nguyen, H.-j. Zhang, H. H. S. Fong, S. G. Franzblau, D. D. Soejarto and M. V. Chau, J. Nat. Prod., 2011, 74, 1318-1322.

74 D. Wakana, N. Kawahara and Y. Goda, J. Nat. Med., 2011, 65, 18-23.

75 I. M. Isaev and M. I. Isaev, Chem. Nat. Compd., 2011, 47, 587-591.

76 J. Linnek, A.-C. Mitaine-Offer, T. Miyamoto, C. Tanaka, T. Paululat, S. Avunduk, Ö. Alankuş-Çalişkan and M.-A. Lacaille-Dubois, Helv. Chim. Acta, 2011, 94, 230-237.

77 H. Kuang, Y. Su, B. Yang, Y. Xia, Q. Wang, Z. Wang and Z. Yu, Molecules, 2011, 16, 4348-4357.

78 T. K. Naubeev, K. K. Uteniyazov and M. I. Isaev, Chem. Nat. Compd., 2011, 47, 250-253.

79 Z. Tian, Y. Sun, P. Xiao and E. Wu, Recent Prog. Med. Plants, 2011, 31, 49-63.

80 M. Liu, M. Gan, S. Lin, Y. Zhang, J. Zi, W. Song, X. Fan, Y. Liu, Y. Yang and J. Shi, Org. Lett., 2011, 13, 2856-2859.

81 M. Gan, M. Liu, B. Liu, S. Lin, Y. Zhang, J. Zi, W. Song, F. Ye, X. Chen and J. Shi, J. Nat. Prod., 2011, 74, 2431-2437.

82 K. L. Lang, T. de R. Guimarães, V. R. Machado, L. A. Zimmermann, I. T. Silva, M. R. Teixeira, F. J. Duran, J. A. Falermo, C. M. O. Simões, M. S. B. Caro and E. P. Schenkel, Planta Med., 2011, 77, 1648-1651.

83 K.-W. Lin, S.-C. Yang and C.-N. Lin, Food Chem., 2011, 127, 609-614.

84 C. Hsu, C.-L. Hsieh, Y.-H. Kuo and C.-j. Huang, J. Agric. Food Chem., 2011, 59, 4553-4561.

85 J.-Q. Cao, Y. Zhang, J.-M. Cui and Y.-Q. Zhao, Chin. Chem. Lett., 2011, 22, 583-586.

86 H. M. Ekramul, A. M. Badrul and H. M. Sarowar, Int. J. Pharm. Sci. Res., 2011, 2, 1135-1146.

87 N. Li, C.-F. Wu, X.-Y. Xu, Z.-Y. Liu, X. Li and Y.-Q. Zhao, Eur. J. Med. Chem., 2012, 50, 173-178.

88 X. Li, J. Q. Cao, L. Shi and Y. Q. Zhao, Chin. Chem. Lett., 2011, 22, 1461-1464.

89 R. Grougnet, P. Magiatis, S. Mitaku, A.-L. Skaltsounis, P. Cabalion, F. Tillequin and S. Michel, Helv. Chim. Acta, 2011, 94, 656-661.

90 J.-M. Zhao, N. Li, H. Zhang, C.-f. Wu, H.-R. Piao and Y.-Q. Zhao, Bioorg. Med. Chem. Lett., 2011, 21, 1027-1031.
91 J. Xiong, M. Taniguchi, Y. Kashiwada, T. Yamagishi and Y. Takaishi, J. Nat. Med., 2011, 65, 217-223.

92 T. Nuanyai, R. Sappapan, T. Vilaivan and K. Pudhom, Phytochem. Lett., 2011, 4, 183-186.

93 T. Asai and Y. Fujimoto, Phytochem. Lett., 2011, 4, 3842.

94 W. Ding, F. Zeng, L. Xu, Y. Chen, Y. Wang and X. Wei, J. Nat. Prod., 2011, 74, 1868-1874.

95 G.-Y. Zhu, Y.-W. Li, D. K.-P. Hau, Z.-H. Jiang, Z.-L. Yu and W.-F. Fong, J. Agric. Food Chem., 2011, 59, 200-205.

96 H.-H. Chan, T.-L. Hwang, M. V. B. Reddy, D.-T. Li, K. Qian, K. F. Bastow, K.-H. Lee and T.-S. Wu, J. Nat. Prod., 2011, 74, 796-802.

97 L.-W. Qi, C.-Z. Wang and C.-S. Yuan, Phytochemistry, 2011, 72, 689-699.

98 K. Radad, R. Moldzio and W.-D. Rausch, CNS Neurosci. Ther., 2011, 17, 761-767.

99 M.-G. Phan, T.-T. C. Truong, T.-S. Phan, K. Matsunami and H. Otsuka, Phytochem. Lett., 2011, 4, 179-182.

100 X.-X. Weng, Y. Shao, Y.-Y. Chen, W. Gao, L. Cheng and D.-Y. Kong, J. Asian Nat. Prod. Res., 2011, 13, 749-755.

101 G.-Y. Zhu, Y.-W. Li, D. K.-P. Hau, Z.-H. Jiang, Z.-L. Yu and W.-F. Fong, Chem. Biodiversity, 2011, 8, 1853-1863.

102 S.-J. Qu, J.-J. Tan, J.-G. Cai, Y.-P. Ling, S.-F. Zhang, C.-H. Tan and D.-Y. Zhu, J. Asian Nat. Prod. Res., 2011, 13, 178-181.

103 J. H. Kim and Y. N. Han, Phytochemistry, 2011, 72, 14531459.

104 Q. Liu, J.-J. Lv, M. Xu, D. Wang, H.-T. Zhu, C.-R. Yang and Y.-J. Zhang, Nat. Prod. Bioprospect., 2011, 1, 124-128.

105 J.-P. Liu, D. Lu and P.-Y. Li, J. Asian Nat. Prod. Res., 2011, 13, 198-204.

106 M. Zhou, M. Xu, D. Wang, H.-T. Zhu, C.-R. Yang and Y.-J. Zhang, Helv. Chim. Acta, 2011, 94, 2010-2019.

107 L. Shi, J.-Q. Cao, S.-M. Shi and Y.-Q. Zhao, J. Asian Nat. Prod. Res., 2011, 13, 168-177.

108 J.-R. Wang, H.-L. Liu, T. Kurtán, A. Mándi, S. Antus, J. Li, H.-Y. Zhang and Y.-W. Guo, Org. Biomol. Chem., 2011, 9, 7685-7696.

109 J. Hu, X. Wang and X. Shi, Eur. J. Org. Chem., 2011, 2011, 7215-7223.

110 Y. Zhang, J.-S. Wang, J. Luo and L.-Y. Kong, Chem. Pharm. Bull., 2011, 59, 282-286.

111 H.-L. Huang, C.-M. Wang, Z.-H. Wang, M.-J. Yao, G.-T. Han, J.-C. Yuan, K. Gao and C.-S. Yuan, J. Nat. Prod., 2011, 74, 2235-2242.

112 J. Wang, Y. Zhang, J. Luo and L. Kong, Magn. Reson. Chem., 2011, 49, 450-457.

113 J.-S. Wang, Y. Zhang, D.-D. Wei, X.-B. Wang, J. Luo and L.-Y. Kong, Chem. Biodiversity, 2011, 8, 2025-2034.

114 K. H. Kim, S. U. Choi, Y. C. Kim and K. R. Lee, J. Nat. Prod., 2011, 74, 54-59.

115 X.-J. Zhou, M. Xu, X.-S. Li, Y.-H. Wang, Y. Gao, R. Cai and Y.-X. Cheng, Bull. Korean Chem. Soc., 2011, 32, 127-130.

116 F. Wang, Z.-L. Li, H.-H. Cui, H.-M. Hua, Y.-K. Jing and S.-W. Liang, J. Asian Nat. Prod. Res., 2011, 13, 193-197.

117 S. A. Shaheen, Z. M. H. Abu, I. K. Nazer, R. M. Darwish and H. I. Al-Jaber, Nat. Prod. Res., 2011, 25, 1312-1318. 
118 J. X. Chen, J. C. Chen, Y. Sun, Y. X. Yan, L. M. Kong, Y. Li and M. H. Qiu, Planta Med., 2011, 77, 1844-1847.

119 H.-T. Zhong, F. Li, B. Chen and M.-K. Wang, Helv. Chim. Acta, 2011, 94, 2061-2065.

120 F. U. Khan, J. Hussain, I. U. Khan, R. Ullah, I. Ali, Z. Muhammad, H. Hussain and M. R. Shah, Chem. Nat. Compd., 2011, 47, 234-236.

121 S.-B. Wu, Q.-Y. Bao, W.-X. Wang, Y. Zhao, G. Xia, Z. Zhao, H. Zeng and J.-F. Hu, Planta Med., 2011, 77, 922-928.

122 S.-i. Kurimoto, Y. Kashiwada, K.-H. Lee and Y. Takaishi, Phytochemistry, 2011, 72, 2205-2211.

123 X. Fang, Y. T. Di and X. J. Hao, Curr. Org. Chem., 2011, 15, 1363-1391.

124 B. M. Komane, E. I. Olivier and A. M. Viljoen, Phytochem. Lett., 2011, 4, 1-9.

125 B. Heasley, Eur. J. Org. Chem., 2011, 19-46.

126 T. Mayanti, R. Tjokronegoro, U. Supratman, M. R. Mukhtar, K. Awang and A. H. A. Hadi, Molecules, 2011, 16, 2785-2795.

127 I. A. Najmuldeen, A. H. A. Hadi, K. Awang, K. Mohamad, K. A. Ketuly, M. R. Mukhtar, S.-L. Chong, G. Chan, M. A. Nafiah, N. S. Weng, O. Shirota, T. Hosoya, A. E. Nugroho and H. Morita, J. Nat. Prod., 2011, 74, 1313-1317.

128 X.-H. Yan, Y.-T. Di, X. Fang, S.-Y. Yang, H.-P. He, S.-L. Li, Y. Lu and X.-J. Hao, Phytochemistry, 2011, 72, 508-513.

129 Y. Zhang, J.-S. Wang, X.-B. Wang, D.-D. Wei, J.-G. Luo, J. Luo, M.-H. Yang and L.-Y. Kong, Tetrahedron Lett., 2011, 52, 2590-2593.

130 S.-P. Yang, H.-D. Chen, S.-G. Liao, B.-J. Xie, Z.-H. Miao and J.-M. Yue, Org. Lett., 2011, 13, 150-153.

131 C. P. Wong, M. Shimada, Y. Nagakura, A. E. Nugroho, Y. Hirasawa, T. Kaneda, K. Awang, A. H. A. Hadi, K. Mohamad, M. Shiro and H. Morita, Chem. Pharm. Bull., 2011, 59, 407-411.

132 Z.-S. Su, S.-P. Yang, S. Zhang, L. Dong and J.-M. Yue, Helv. Chim. Acta, 2011, 94, 1515-1526.

133 J.-L. Yang, L.-L. Liu and Y.-P. Shi, Planta Med., 2011, 77, 271-276.

134 C. A. C. Barrera, E. D. C. Barrera, D. S. G. Falla, G. D. Murcia and L. E. C. Suarez, Chem. Pharm. Bull., 2011, 59, 855-859.

135 J. Liu, S.-P. Yang, Z.-S. Su, B.-D. Lin, Y. Wu and J.-M. Yue, Phytochemistry, 2011, 72, 2189-2196.

136 F. Zhang, S.-G. Liao, C.-R. Zhang, X.-F. He, W.-S. Chen and J.-M. Yue, Planta Med., 2011, 77, 1617-1622.

137 T. K. Nsiama, H. Okamura, T. Hamada, Y. Morimoto, M. Doe, T. Iwagawa and M. Nakatani, Phytochemistry, 2011, 72, 1854-1858.

138 W. Ravangpai, D. Sommit, T. Teerawatananond, N. Sinpranee, T. Palaga, S. Pengpreecha, N. Muangsin and K. Pudhom, Bioorg. Med. Chem. Lett., 2011, 21, 44854489.

139 J.-F. Hu, H. Fan, L.-J. Wang, S.-B. Wu and Y. Zhao, Phytochem. Lett., 2011, 4, 292-297.

140 H.-B. Liu, C.-R. Zhang, S.-H. Dong, L. Dong, Y. Wu and J.-M. Yue, Chem. Pharm. Bull., 2011, 59, 1003-1007.

141 J. Luo, J.-S. Wang, J.-G. Luo, X.-B. Wang and L.-Y. Kong, Tetrahedron, 2011, 67, 2942-2948.
142 J. Luo, J.-S. Wang, X.-B. Wang, J.-G. Luo and L.-Y. Kong, Chem. Pharm. Bull., 2011, 59, 225-230.

143 J. Luo, Y. Li, J.-S. Wang and L.-Y. Kong, Chem. Biodiversity, 2011, 8, 2261-2269.

144 Y. Li, J. Luo, Q. Wang and L.-Y. Kong, J. Asian Nat. Prod. Res., 2011, 13, 781-786.

145 J.-L. Yin, Y.-T. Di, X. Fang, E.-D. Liu, H.-Y. Liu, H.-P. He, S.-L. Li, S.-F. Li and X.-J. Hao, Tetrahedron Lett., 2011, 52, 3083-3085.

146 J. Luo, J.-S. Wang, W.-J. Cao and L.-Y. Kong, Zhongguo Tianran Yaowu, 2011, 9, 98-100.

147 B.-D. Lin, C.-R. Zhang, S.-P. Yang, Y. Wu and J.-M. Yue, Chem. Pharm. Bull., 2011, 59, 458-465.

148 Q. Zhang, Y.-T. Di, H.-P. He, X. Fang, D.-L. Chen, X.-H. Yan, F. Zhu, T.-Q. Yang, L.-L. Liu and X.-J. Hao, J. Nat. Prod., 2011, 74, 152-157.

149 Y. Tanaka, T. Yamada, Y. In, O. Muraoka, T. Kajimoto and R. Tanaka, Tetrahedron, 2011, 67, 782-792.

150 J. Li, M.-Y. Li, T. Satyanandamurty and J. Wu, Helv. Chim. Acta, 2011, 94, 1651-1656.

151 W.-H. Jiao, H. Gao, F. Zhao, F. He, G.-X. Zhou and X.-S. Yao, Chem. Biodiversity, 2011, 8, 1163-1169.

152 M. Zhao, S. T. Lau, X. Q. Zhang, W. C. Ye, P. S. Leung, C.-T. Che and Z.-X. Lin, Helv. Chim. Acta, 2011, 94, 20992105.

153 H. Chen, J. Bai, Z.-F. Fang, S.-S. Yu, S.-G. Ma, S. Xu, Y. Li, J. Qu, J.-H. Ren, L. Li, Y.-K. Si and X.-G. Chen, J. Nat. Prod., 2011, 74, 2438-2445.

154 T. Diyabalanage, R. Ratnayake, J. A. Wilson, C. J. Henrich, J. A. Beutler, N. H. Colburn, J. B. McMahon and K. R. Gustafson, Bioorg. Med. Chem. Lett., 2011, 21, 43974399.

155 H. R. Siddique and M. Saleem, Life Sci., 2011, 88, 285-293. 156 C. Gauthier, J. Legault, M. Piochon-Gauthier and A. Pichette, Phytochem. Rev., 2011, 10, 521-544.

157 J. Shinozaki, T. Nakane, N. Onodera, A. Takano and K. Masuda, Chem. Pharm. Bull., 2011, 59, 767-769.

158 Y.-P. Liu, X.-H. Cai, T. Feng, Y. Li, X.-N. Li and X.-D. Luo, J. Nat. Prod., 2011, 74, 1161-1168.

159 X.-F. He, X.-N. Wang, S. Yin, L. Dong and J.-M. Yue, Bioorg. Med. Chem. Lett., 2011, 21, 125-129.

160 N.-Y. Yang, J.-H. Chen, G.-S. Zhou, Y.-P. Tang, J.-A. Duan, L.-J. Tian and X.-H. Liu, Fitoterapia, 2011, 82, 927-931.

161 M. H. Kazmi, I. Fatima, A. Malik, L. Iqbal, M. Latif and N. Afza, J. Asian Nat. Prod. Res., 2011, 13, 1081-1086.

162 T. Morikawa, H. Oominami, H. Matsuda and M. Yoshikawa, J. Nat. Med., 2011, 65, 129-134.

163 Y.-E. Guo, L.-L. Wang, Z.-L. Li, S.-L. Niu, X.-Q. Liu, H.-M. Hua, H. Chen, J. Chu and T.-C. Zhang, J. Asian Nat. Prod. Res., 2011, 13, 440-443.

164 X. Wang, E. Habib, F. Leon, M. M. Radwan, N. Tabanca, J. Gao, D. E. Wedge and S. J. Cutler, Chem. Biodiversity, 2011, 8, 2331-2340.

165 X.-L. Wang, A.-E. Hay, A. Matheeussen, M. P. Gupta and K. Hostettmann, Magn. Reson. Chem., 2011, 49, 184-189.

166 S. Zhao, Z. Huang and J. Gao, Bull. Korean Chem. Soc., 2011, 32, 1368-1370. 
167 Z. Shu, Z. Chen, X.-j. Ding, B.-q. Lu, C.-j. Ji, Q.-m. Xu, X.-r. Li and S.-l. Yang, Heterocycles, 2011, 83, 2365-2372.

168 P. Pailee, V. Prachyawarakorn, C. Mahidol, S. Ruchirawat and P. Kittakoop, Eur. J. Org. Chem., 2011, 2011, 3809-3814.

169 M. Lee, M. K. Lee, Y. C. Kim and S. H. Sung, Bioorg. Med. Chem. Lett., 2011, 21, 2906-2910.

170 S. V. Fannang, V. Kuete, C. D. Mbazoa, J. I. Momo, H. T. Van-Dufat, F. Tillequin, E. Seguin, E. Chosson and J. Wandji, Chem. Nat. Compd., 2011, 47, 404-407.

171 A. Yokosuka, S. Kawakami, M. Haraguchi and Y. Mimaki, Phytochem. Lett., 2011, 4, 259-266.

172 R. A. S. Macahig, K. Matsunami and H. Otsuka, Chem. Pharm. Bull., 2011, 59, 397-401.

173 D. N. Vedernikov and V. I. Roshchin, Khim. Rastit. Syr'ya, 2011, 95-102.

174 T. H. Quang, T. T. N. Nguyen, C. V. Minh, P. V. Kiem, X. N. Nguyen, B. H. Tai, P. T. Nguyen, H. T. Nguyen, S. B. Song and Y. H. Kim, J. Nat. Prod., 2011, 74, 1908-1915.

175 B. K. Ponou, R. B. Teponno, M. Ricciutelli, T. B. Nguelefack, L. Quassinti, M. Bramucci, G. Lupidi, L. Barboni and L. A. Tapondjou, Chem. Biodiversity, 2011, 8, 1301-1309.

176 R. Badoni, D. K. Semwal, U. Rawat and M. S. M. Rawat, Helv. Chim. Acta, 2011, 94, 464-473.

177 R. B. Semwal, D. K. Semwal, R. Semwal, R. Singh and M. S. M. Rawat, J. Ethnopharmacol., 2011, 135, 78-87.

178 T. Mencherini, P. Picerno, M. Festa, P. Russo, A. Capasso and R. Aquino, J. Nat. Prod., 2011, 74, 2116-2121.

179 Y. Qu, J. Liang and X. Feng, Tianran Chanwu Yanjiu Yu Kaifa, 2011, 23, 577-581.

180 Z. Zhang, C. Zhao, S. Chen and H. Ji, Yaoxue Jinzhan, 2011, 35, 353-359.

181 S.-Y. Cheng, C.-M. Wang, Y.-M. Hsu, T.-J. Huang, S.-C. Chou, E.-H. Lin and C.-H. Chou, J. Nat. Prod., 2011, 74, 1744-1750, 2030.

182 D.-Q. Luo, H.-Y. Deng, X.-L. Yang, B.-Z. Shi and J.-Z. Zhang, Helv. Chim. Acta, 2011, 94, 1041-1047.

183 X.-F. Niu, X. Liu, L. Pan and L. Qi, Fitoterapia, 2011, 82, 960963.

184 B. M. Mba'ning, B. N. Lenta, S. Ngouela, D. T. Noungoue, F. Tantangmo, F. M. Talontsi, E. Tsamo and H. Laatsch, Z. Naturforsch., B: J. Chem. Sci., 2011, 66, 1270-1274.

185 P. Liu, P. Hu, R.-X. Deng, R. Li, L. Yang and W.-P. Yin, Helv. Chim. Acta, 2011, 94, 136-141.

186 C. Y. Ragasa, D. L. Espineli and C.-C. Shen, Chem. Pharm. Bull., 2011, 59, 778-782.

187 X.-Q. Chen, Y. Li, J. He, X. Cheng, K. Wang, M.-M. Li, Z.-H. Pan, L.-Y. Peng and Q.-S. Zhao, Chem. Pharm. Bull., 2011, 59, 496-498.

188 N. Zeng, Y. Shen, L.-Z. Li, W.-H. Jiao, P.-Y. Gao, S.-J. Song, W.-S. Chen and H.-W. Lin, J. Nat. Prod., 2011, 74, 732-738.

189 H. Yang, E. J. Jeong, J. Kim, S. H. Sung and Y. C. Kim, J. Nat. Prod., 2011, 74, 751-756.

190 C.-B. Xue, D.-W. Chai, X.-J. Jin, Y.-R. Bi, X.-J. Yao, W.-S. Wu and Y. Zhu, Phytochemistry, 2011, 72, 1804-1813.

191 M. Shaaban, K. A. Shaaban, H. I. Abd-Alla, A. G. Hanna and H. Laatsch, Z. Naturforsch., B: J. Chem. Sci., 2011, 66, 425432.
192 Y.-L. Huang, T. Tsujita, T. Tanaka, Y. Matsuo, I. Kouno, D.-P. Li and G.-i. Nonaka, Phytochemistry, 2011, 72, 20062014.

193 X.-Q. Liu, H.-L. Huang, M.-J. Yao, G.-T. Han, N. Liu, J.-C. Yuan and C.-S. Yuan, Helv. Chim. Acta, 2011, 94, 2264-2271.

194 M. J. Nunez, M. L. Kennedy, I. A. Jimenez and I. L. Bazzocchi, Tetrahedron, 2011, 67, 3030-3033.

195 J.-G. Luo, W. Nie and L.-Y. Kong, J. Asian Nat. Prod. Res., 2011, 13, 529-533.

196 D. Liang, Z.-Y. Hao, G.-J. Zhang, Q.-J. Zhang, R.-Y. Chen and D.-Q. Yu, J. Nat. Prod., 2011, 74, 2128-2136.

197 X.-A. Huang, X.-L. Shen, Y.-J. Hu, Y.-M. Liu, K.-L. Liu, F.-X. Zhang and X.-X. Zhou, Molecules, 2011, 16, 8076-8082.

198 T. Varughese, M. M. Manir, M. Rahaman, J. K. Kim, B.-G. Lee and S.-S. Moon, Planta Med., 2011, 77, 2029-2036.

199 K. Matsunami, H. Otsuka and Y. Takeda, Chem. Pharm. Bull., 2011, 59, 1274-1280.

200 T. Nakano, S. Sugimoto, K. Matsunami and H. Otsuka, Chem. Pharm. Bull., 2011, 59, 1141-1148.

201 S. Okazaki, K. Kinoshita, S. Ito, K. Koyama, H. Yuasa and K. Takahashi, Phytochemistry, 2011, 72, 136-146.

202 L. Gao, L. Zhang, N. Li, J.-Y. Liu, P.-l. Cai and S.-l. Yang, Carbohydr. Res., 2011, 346, 2881-2885.

203 L. O. A. Manguro, J. O. Midiwo, L. F. Tietze and P. Hao, ARKIVOC, 2011, (ii), 172-198.

204 Y. Nakahara, M. Okawa, J. Kinjo and T. Nohara, Chem. Pharm. Bull., 2011, 59, 1329-1339.

205 K. H. Kim, I. K. Lee, S. U. Choi, J. H. Lee, E. Moon, S. Y. Kim and K. R. Lee, Planta Med., 2011, 77, 1555-1558.

206 W. Dong, X. Liu, X. Li, D. Yang and L. Ding, Fitoterapia, 2011, 82, 782-785.

207 K. Foubert, F. Cuyckens, A. Matheeussen, A. Vlietinck, S. Apers, L. Maes and L. Pieters, Phytochemistry, 2011, 72, 1414-1423.

208 D.-L. Liu, N.-L. Wang, X. Zhang and X.-S. Yao, Helv. Chim. Acta, 2011, 94, 693-702.

209 D. Liu, X. Zhang, S. Wang, N. Wang and X. Yao, Chin. Chem. Lett., 2011, 22, 957-960.

210 A. Rubio-Moraga, G. J. Gerwig, N. Castro-Diaz, M. L. Jimeno, J. Escribano, J.-A. Fernandez and J. P. Kamerling, Ind. Crops Prod., 2011, 34, 1401-1409.

211 H. T. Nguyen, H. Q. Tran, T. T. N. Nguyen, V. M. Chau, K. A. Bui, Q. L. Pham, M. C. Nguyen and Y. H. Kim, Chem. Pharm. Bull., 2011, 59, 1417-1420.

212 E. P. Mazzola, A. Parkinson, E. J. Kennelly, B. Coxon, L. S. Einbond and D. I. Freedberg, Carbohydr. Res., 2011, 346, 759-768.

213 G.-L. Jin, C.-J. Zheng, W.-B. Xin, Z.-J. Mao, P.-X. Sun, Z.-X. Zeng and L.-P. Qin, Arch. Pharmacal Res., 2011, 34, 869-873.

214 S. Yao, J.-G. Luo, L. Ma and L.-Y. Kong, Zhongguo Tianran Yaowu, 2011, 9, 401-405.

215 G. Gao, Z. Lu, S. Tao, S. Zhang and F. Wang, Carbohydr. Res., 2011, 346, 2200-2205.

216 G.-C. Gao, Z.-X. Lu, S.-H. Tao, S. Zhang, F.-Z. Wang and Q.-X. Li, Can. J. Chem., 2011, 89, 1277-1282. 
217 Q. Xue, Z.-L. Sun, M.-L. Guo, Y. Wang, G. Zhang and X.-K. Wang, Nat. Prod. Res., 2011, 25, 772-780.

218 Q. Wu, Y. Wang and M. Guo, Chem. Pharm. Bull., 2011, 59, 666-671.

219 Z. Ali and I. A. Khan, Phytochemistry, 2011, 72, 2075-2080.

220 A. F. Awantu, B. N. Lenta, T. Bogner, Y. F. Fongang, S. Ngouela, J. D. Wansi, E. Tsamo and N. Sewald, $Z$. Naturforsch., B: J. Chem. Sci., 2011, 66, 624-628.

221 J.-J. Liu, X.-L. Wang, B.-L. Guo, W.-H. Huang, P.-G. Xiao, C.-Q. Huang, L.-Z. Zheng, G. Zhang, L. Qin and G.-Z. Tu, J. Asian Nat. Prod. Res., 2011, 13, 851-860.

222 X. N. Nguyen, H. Y. Lim, P. V. Kiem, C. V. Minh, V. K. Thu, B. H. Tai, T. H. Quang, S. B. Song and Y. H. Kim, Bioorg. Med. Chem. Lett., 2011, 21, 6143-6147.

223 J. He, J. Ma, D.-W. Lai, Y.-m. Zhang and W.-J. Sun, Nat. Prod. Res., 2011, 25, 1771-1775.

224 H.-Z. Fu, C.-J. Li, J.-Z. Yang, Z.-F. Shen and D.-M. Zhang, J. Nat. Prod., 2011, 74, 1066-1072.

225 Z. A. Kozhamkulova, M. M. Radwan, G. E. Zhusupova, Z. A. Abilov and S. A. Ross, Phytochem. Lett., 2011, 4, 323327.

226 T. K. Tabopda, A.-C. Mitaine-Offer, T. Miyamoto, C. Tanaka, J.-F. Mirjolet, O. Duchamp, B. T. Ngadjui and M.-A. LacailleDubois, Helv. Chim. Acta, 2011, 94, 2066-2076.

227 Y. Chen, Y. Zhao, M. Wang, H. Sun, Y. Dong and X. Feng, Chem. Nat. Compd., 2011, 47, 940-943.

228 Y.-X. He, L. Li, K. Zhang and Z.-R. Liu, J. Asian Nat. Prod. Res., 2011, 13, 1104-1109.

229 T. K. Tabopda, A.-C. Mitaine-Offer, T. Miyamoto, C. Tanaka, B. T. Ngadjui, J.-F. Mirjolet, O. Duchamp and M.-A. LacailleDubois, Helv. Chim. Acta, 2011, 94, 914-922.

230 Z.-Q. Cheng, D. Yang, Q.-Y. Ma, X.-H. Yi, N.-L. Zhang, J. Zhou and Y.-X. Zhao, Bull. Korean Chem. Soc., 2011, 32, 1403-1406.

231 C.-J. Li, J.-Z. Yang, S.-S. Yu, D.-M. Zhang, W. Xue, Y.-H. Yuan and N.-H. Chen, Zhongguo Tianran Yaowu, 2011, 9, 321328.

232 L. A. Tapondjou, L. B. T. Nyaa, P. Tane, M. Ricciutelli, L. Quassinti, M. Bramucci, G. Lupidi, B. K. Ponou and L. Barboni, Carbohydr. Res., 2011, 346, 2699-2704.

233 W.-W. Fu, J.-N. Fu, W.-M. Zhang, L.-X. Sun, Y.-H. Pei and P. Liu, Molecules, 2011, 16, 4371-4378.

234 Z. Zhang, X. Fang, Y.-H. Wang, G.-M. Liu, H. Xiao, X.-J. Hao and H.-P. He, J. Asian Nat. Prod. Res., 2011, 13, 838-844.

235 S. Sugimoto, K. Matsunami and H. Otsuka, Chem. Pharm. Bull., 2011, 59, 466-471.

236 N. B. Sarikahya, M. Pekmez, N. Arda, P. Kayce, N. U. K. Yavasoglu and S. Kirmizigul, Phytochem. Lett., 2011, 4, 415-420.

237 A. Sosa, C. Rosquete, L. Rojas, L. Pouysegu, S. Quideau, T. Paululat, A.-C. Mitaine-Offer and M.-A. Lacaille-Dubois, Helv. Chim. Acta, 2011, 94, 1850-1859.

238 H. Zhang, A. K. Samadi, K. V. Rao, M. S. Cohen and B. N. Timmermann, J. Nat. Prod., 2011, 74, 477-482.

239 Y. Ding, H.-F. Tang, J.-B. Wang, D. Liu, X.-R. Tian, X.-Y. Wang and X.-M. Zhou, Biochem. Syst. Ecol., 2011, 39, 236-239.
240 X.-Y. Wang, X.-L. Chen, H.-F. Tang, H. Gao, X.-R. Tian and P.-H. Zhang, Planta Med., 2011, 77, 1550-1554.

241 Q.-H. Wang, J. Zhang, X. Ma, X.-Y. Ye, B.-Y. Yang, Y.-G. Xia and H.-X. Kuang, Zhongguo Tianran Yaowu, 2011, 9, 17-21.

242 G. Timite, A.-C. Mitaine-Offer, T. Miyamoto, C. Tanaka, J.-F. Mirjolet, O. Duchamp and M.-A. Lacaille-Dubois, Phytochemistry, 2011, 72, 503-507.

243 T. Morikawa, X. Li, E. Nishida, S. Nakamura, K. Ninomiya, H. Matsuda, M. Hamao, O. Muraoka, T. Hayakawa and M. Yoshikawa, Chem. Pharm. Bull., 2011, 59, 889-895.

244 K. Yang, Y. Li, L. Ge and Z. Qin, Adv. Mater. Res. (DurntenZurich, Switz.), 2011, 236-238, 1731-1737.

245 M. Tene, P. Chabert, O. Note, T. J. N. Kenla, P. Tane and A. Lobstein, Phytochem. Lett., 2011, 4, 89-92.

246 J.-G. Luo, X. Chen and L.-Y. Kong, Chem. Pharm. Bull., 2011, 59, 518-521.

247 D. Du, L. Fang, J. Qu, S. Yu, S. Ma, H. Lv, J. Liu, Y. Liu, J. Wang and X. Wang, Planta Med., 2011, 77, 1631-1638.

248 S. Y. Lee, J. S. Kim, S. H. Shim and S. S. Kang, Bull. Korean Chem. Soc., 2011, 32, 3650-3654.

249 Q. Wen, D. Yuan, K.-H. Xie, T.-Z. Cai and H.-Z. Fu, J. Asian Nat. Prod. Res., 2011, 13, 869-878.

250 Q. Chen, J.-G. Luo and L.-Y. Kong, Carbohydr. Res., 2011, 346, 2206-2212.

251 N. A. Khan, Nat. Prod. Res., 2011, 25, 1687-1694.

252 A. Tava, L. Pecetti, M. Romani, M. Mella and P. Avato, J. Agric. Food Chem., 2011, 59, 6142-6149.

253 B. Hernandez-Carlos, A. Gonzalez-Coloma, A. U. OrozcoValencia, M. V. Ramirez-Mares, M. F. Andres-Yeves and P. Joseph-Nathan, Phytochemistry, 2011, 72, 743-751.

254 Y.-X. Zhao, W.-J. Liang, H.-J. Fan, Q.-Y. Ma, W.-X. Tian, H.-F. Dai, H.-Z. Jiang, N. Li and X.-F. Ma, Carbohydr. Res., 2011, 346, 1302-1306.

255 W.-J. Liang, Q.-Y. Ma, H.-Z. Jiang, J. Zhou, J. Pang and Y.-X. Zhao, Chem. Nat. Compd., 2012, 47, 935-939.

256 X.-m. Song, Y. Liu and B.-c. Cai, Shenyang Yaoke Daxue Xuebao, 2010, 27, 627-629, 647.

257 M. Z. Getiya, M. A. Gabelaya, V. D. Mshvildadze, A. Pichette, S. Lavoie and G. E. Dekanosidze, Chem. Nat. Compd., 2011, 47, 764-766.

258 A. I. Hamed, M. Masullo, M. G. Sheded, U. A. Mahalel, M. M. Tawfik, A. Perrone and S. Piacente, Phytochem. Lett., 2011, 4, 353-356.

259 H.-X. Kuang, H.-W. Li, Q.-H. Wang, B.-Y. Yang, Z.-B. Wang and Y.-G. Xia, Molecules, 2011, 16, 4642-4651.

260 J.-S. Jiang, Z.-Z. Liu, Z.-M. Feng, Y.-N. Yang and P.-C. Zhang, J. Asian Nat. Prod. Res., 2011, 13, 276-280.

261 I. L. Acebey-Castellon, L. Voutquenne-Nazabadioko, D. T. M. Huong, N. Roseau, N. Bouthagane, D. Muhammad, M. D. E. Le, S. C. Gangloff, M. Litaudon, T. Sevenet, V. H. Nguyen and C. Lavaud, J. Nat. Prod., 2011, 74, 163-168.

262 R.-X. Deng, W.-L. Duan, P. Liu, Y.-L. Yang and W.-P. Yin, J. Asian Nat. Prod. Res., 2011, 13, 230-237.

263 V. T. T. Thanh, V. C. Pham, H. H. Nguyen, H. D. T. Mai, H. N. T. Minh, V. H. Nguyen, M. Litaudon, F. Gueritte and V. M. Chau, Eur. J. Org. Chem., 2011, 2011, 4108-4111. 
264 M. A. Tantry, R. Khan, S. Akbar, A. R. Dar, A. S. Shawl and M. S. Alam, Chin. Chem. Lett., 2011, 22, 575-579.

265 Y.-P. Ma, N. Li, J. Gao, K.-L. Fu, Y. Qin, G.-Y. Li and J.-H. Wang, Helv. Chim. Acta, 2011, 94, 1881-1887.

266 Q.-X. Wu, Y.-B. Su and Y. Zhu, Fitoterapia, 2011, 82, 493-496.

267 P. Somwong, R. Suttisri and A. Buakeaw, Fitoterapia, 2011, 82, 1047-1051.

268 F. C. Silva, V. G. Rodrigues, L. P. Duarte, G. D. F. Silva, R. R. S. Miranda and S. A. V. Filho, J. Chem. Res., 2011, 35, 555-557.

269 F. C. Silva, L. P. Duarte, G. D. F. Silva, S. A. V. Filho, I. S. Lula, J. A. Takahashi and W. S. T. Sallum, J. Braz. Chem. Soc., 2011, 22, 943-949.

270 S. V. Fannang, V. Kuete, C. M. Djama, M. D. J. Dongfack, J. D. Wansi, F. Tillequin, E. Seguin, E. Chosson and J. Wandji, Chin. Chem. Lett., 2011, 22, 171-174.

271 G. Li, D. Wang and S. Xu, Nat. Prod. Res., 2011, 25, 136-140.

272 M. L. Kennedy, G. G. Llanos, S. Castanys, F. Gamarro, I. L. Bazzocchi and I. A. Jimenez, Chem. Biodiversity, 2011, 8, 2291-2298.

273 G. Chen, H. Ren and C. Yu, Chem. Nat. Compd., 2011, 47, 918-920.

274 S. Iqbal, A. Khan, V. U. Ahmad, M. A. Khan, S. Bader, U. Farooq, S. S. Khan, A. Zahoor and R. B. Tareen, Nat. Prod. Commun., 2011, 6, 179-182.

275 W. Zhao, J. X. Pu, X. Du, Y. L. Wu, Y. Zhao, F. He, H. B. Zhang, Y. B. Xue, W. L. Xiao, G. Q. Chen and H. D. Sun, Arch. Pharmacal Res., 2011, 34, 2007-2014.

276 C. Wu, L. Wang, X.-X. Yang, Y.-H. Duan, Y. Dai, R.-W. Jiang, W.-C. Ye and Y.-L. Li, J. Asian Nat. Prod. Res., 2011, 13, 434439.

277 A. Mittal and M. Ali, Int. Res. J. Pharm., 2011, 2(9), 52-54.

278 S. Begum, S. Perwaiz, B. S. Siddiqui, S. Khan, S. Fayyaz and M. Ramzan, Chem. Biodiversity, 2011, 8, 850-861.

279 B.-b. Zhang, K. Shi, Z.-x. Liao, Y. Dai and Z.-h. Zou, Fitoterapia, 2011, 82, 854-860.

280 S. Y. Lee, J. S. Kim, R. J. Choi, Y. S. Kim, J.-H. Lee and S. S. Kang, Chem. Pharm. Bull., 2011, 59, 742-746.

281 K. Mazumder, E. R. O. Siwu, S. Nozaki, Y. Watanabe, K. Tanaka and K. Fukase, Phytochem. Lett., 2011, 4, 287-291.

282 R. T. Kengap, G. D. W. F. Kapche, J.-P. Dzoyem, I. K. Simo, P. Ambassa, L. P. Sandjo, B. M. Abegaz and B. T. Ngadjui, Helv. Chim. Acta, 2011, 94, 2231-2238.

283 G. Topcu, G. Yapar, Z. Turkmen, A. C. Goren, S. Oksuz, J. K. Schilling and D. G. I. Kingston, Phytochem. Lett., 2011, 4, 421-425.

284 T. Sasaki, W. Li, H. Morimura, S. Li, Q. Li, Y. Asada and K. Koike, Chem. Pharm. Bull., 2011, 59, 1396-1399.

285 J. Hu, X.-Q. Chen and Q.-S. Zhao, J. Asian Nat. Prod. Res., 2011, 13, 105-110.

286 Y. Zhang, L.-J. Li, P. Zhang, H.-F. Pi, H.-L. Ruan and J.-Z. Wu, Helv. Chim. Acta, 2011, 94, 2207-2214.
287 L. P. Lin, W. Qu and J. Y. Liang, Chin. Chem. Lett., 2011, 22, 697-700.

288 X. N. Nguyen, B. H. Tai, T. H. Quang, P. V. Kiem, C. V. Minh, H. N. Nguyen, J.-H. Kim, L.-R. Im, Y.-M. Lee and Y. H. Kim, Bioorg. Med. Chem. Lett., 2011, 21, 1777-1781.

289 X.-Y. Xu, X.-H. Yang, S.-Z. Li and Q.-S. Song, J. Asian Nat. Prod. Res., 2011, 13, 1008-1013.

290 W.-J. Zuo, H.-F. Dai, J. Chen, H.-Q. Chen, Y.-X. Zhao, W.-L. Mei, X. Li and J.-H. Wang, Planta Med., 2011, 77, 1835-1840.

291 Y.-Y. Che, N. Li, L. Zhang and P.-F. Tu, Zhongguo Tianran Yaowu, 2011, 9, 22-25.

292 W.-H. Cai, K. Matsunami, H. Otsuka and Y. Takeda, Am. J. Plant Sci., 2011, 2, 609-618.

293 E. Amin, S. S. El-Hawary, M. M. Fathy, R. Mohammed, Z. Ali, N. Tabanca, D. E. Wedge, J. J. Becnel and I. A. Khan, Planta Med., 2011, 77, 488-491.

294 C. L. Lencina, C. M. C. de, I. Zancanaro, G. Gosmann, V. S. Pires, P. Sonnet, D. Guillaume and E. P. Schenkel, Quim. Nova, 2011, 34, 222-225.

295 X. Cheng, J. Qin, Q. Zeng, S. Zhang, F. Zhang, S. Yan, H. Jin and W. Zhang, Planta Med., 2011, 77, 2061-2065.

296 G. Siedenburg and D. Jendrossek, Appl. Environ. Microbiol., 2011, 77, 3905-3915.

297 R. Badoni, D. K. Semwal, P. P. Badoni, S. K. Kothiyal and U. Rawat, Chin. Chem. Lett., 2011, 22, 81-84.

298 T. Řezanka, L. Siristova, K. Melzoch and K. Sigler, Lipids, 2011, 46, 249-261.

299 M. Isaka, S. Palasarn, S. Supothina, S. Komwijit and J. J. Luangsa-ard, J. Nat. Prod., 2011, 74, 782-789.

300 J.-T. Fan, B. Kuang, G.-Z. Zeng, S.-M. Zhao, C.-J. Ji, Y.-M. Zhang and N.-H. Tan, J. Nat. Prod., 2011, 74, 20692080.

301 Z. Z. Ibraheim, A. S. Ahmed and Y. G. Gouda, Saudi Pharm. J., 2011, 19, 65-74.

302 R. S. T. Kamdem, P. Wafo, S. Yousuf, Z. Ali, A. Adhikari, S. Rasheed, I. A. Khan, B. T. Ngadjui, H.-K. Fun and M. I. Choudhary, Org. Lett., 2011, 13, 5492-5495.

303 C. Abbet, M. Neuburger, T. Wagner, M. Quitschau, M. Hamburger and O. Potterat, Org. Lett., 2011, 13, 13541357.

304 S.-H. Dong, C.-R. Zhang, L. Dong, Y. Wu and J.-M. Yue, J. Nat. Prod., 2011, 74, 1042-1048.

305 M. S. Gachet, O. Kunert, M. Kaiser, R. Brun, M. Zehl, W. Keller, R. A. Muñoz, R. Bauer and W. Schuehly, J. Nat. Prod., 2011, 74, 559-566.

306 N. Tanaka, R. Momose, A. Shibazaki, T. Gonoi, J. Fromont and J.-i. Kobayashi, Tetrahedron, 2011, 67, 6689-6696.

307 J.-H. Lee, K. H. Jang, Y.-J. Lee, H.-S. Lee, C. J. Sim, K.-B. Oh and J. Shin, J. Nat. Prod., 2011, 74, 2563-2570.

308 Y. Hasegawa, X. Gong and C. Kuroda, Nat. Prod. Commun., 2011, 6, 789-792. 